\title{
INTEGRAL/IBIS 17-yr hard X-ray all-sky survey
}

\author{
Roman A. Krivonos, ${ }^{1 \star}$ Sergey Yu. Sazonov, ${ }^{1,2}$ Ekaterina A. Kuznetsova, ${ }^{1}$ \\ Alexander A. Lutovinov, ${ }^{1}$ Ilya A. Mereminskiy, ${ }^{1}$ and Sergey S. Tsygankov ${ }^{1,3}$ \\ ${ }^{1}$ Space Research Institute (IKI), Profsoyuznaya 84/32, Moscow 117997, Russia \\ ${ }^{2}$ Moscow Institute of Physics and Technology, Institutsky per. 9, 141700 Dolgoprudny, Russia \\ ${ }^{3}$ Department of Physics and Astronomy, FI-20014 University of Turku, Finland
}

Accepted 2021 December 21. Received 2021 December 5; in original form 2021 October 25

\begin{abstract}
The International Gamma-Ray Astrophysics Laboratory (INTEGRAL), launched in 2002, continues its successful work in observing the sky at energies $E>20 \mathrm{keV}$. The legacy of the mission already includes a large number of discovered or previously poorly studied hard X-ray sources. The growing INTEGRAL archive allows one to conduct an all-sky survey including a number of deep extragalactic fields and the deepest ever hard X-ray survey of the Galaxy. Taking advantage of the data gathered over 17 years with the IBIS coded-mask telescope of INTEGRAL, we conducted survey of hard X-ray sources, providing flux information from 17 to $290 \mathrm{keV}$. The catalog includes 929 objects, 890 of which exceed a detection threshold of $4.5 \sigma$ and the rest are detected at $4.0-4.5 \sigma$ and belong to known cataloged hard X-ray sources. Among the identified sources of known or suspected nature, 376 are associated with the Galaxy and Magellanic clouds, including 145 low-mass and 115 high-mass X-ray binaries, 79 cataclysmic variables, and 37 of other types; and 440 are extragalactic, including 429 active galactic nuclei (AGNs), 2 ultra-luminous sources, one supernova (AT2018cow) and 8 galaxy clusters. 113 sources remain unclassified. 46 objects are detected in the hard X-ray band for the first time. The $\log N-\log S$ distribution of 356 non-blazar AGNs is measured down to a flux of $2 \times 10^{-12} \mathrm{erg} \mathrm{s}^{-1}$ $\mathrm{cm}^{-2}$ and can be described by a power law with a slope of $1.44 \pm 0.09$ and normalization $8 \times 10^{-3} \mathrm{deg}^{-2}$ at $10^{-11} \mathrm{erg} \mathrm{s}^{-1} \mathrm{~cm}^{-2}$. The $\log N-\log S$ distribution of unclassified sources indicates that the majority of them are of extragalactic origin.
\end{abstract}

Key words: catalogs - surveys - X-rays: general.

\section{INTRODUCTION}

The INTEGRAL (Winkler et al. 2003) observatory has been successfully operating in orbit since its launch in 2002 October. The high sensitivity in the hard X-ray band $(\sim 20-100 \mathrm{keV})$ and relatively good angular resolution of the IBIS coded-mask telescope (Ubertini et al. 2003) makes surveying the hard X-ray sky one of the primary goals of INTEGRAL. Over the past years, INTEGRAL has conducted many surveys, starting with sky areas of a few hundred squared degrees and gradually covering the entire celestial sphere (see Krivonos et al. 2021, for a review).

The INTEGRAL hard X-ray surveys have been used as a basis for studies of various classes of objects, from cataclysmic variables (CVs) and symbiotic stars (see Lutovinov et al. 2020, for a review), through low mass X-ray binaries (LMXB; see Sazonov et al. 2020, for a review) and high mass X-ray binaries (HMXB; see Kretschmar et al. 2019, for a review), to extragalactic objects, mainly active galactic nuclei (AGNs; see Malizia et al. 2020, for a review).

Since 2004, the sky has also been surveyed in hard X-rays by the Burst Alert Telescope (BAT; Barthelmy et al. 2005) of the Neil Gehrels Swift Observatory (Swift; Gehrels et al. 2004), which provides a nearly uniform all-sky coverage (see Oh et al. 2018, and references therein) with somewhat longer exposures at high Galactic latitudes. In contrast to Swift, the INTEGRAL observatory provides

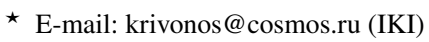

a sky survey with exposures that are deeper near the Galactic plane, which renders the Swift/BAT and INTEGRAL/IBIS hard X-ray surveys complementary to each other.

Krivonos et al. (2007b) presented the first INTEGRAL/IBIS allsky survey based on 3.5 years of observations at the beginning of the mission. The catalog of sources detected in the $17-60 \mathrm{keV}$ energy band comprises 403 objects, 316 of which were found above a threshold of $5 \sigma$ on the time-averaged sky map, and the rest were detected in various subsamples of observations. The most recent data release of the INTEGRAL/IBIS catalog with all-sky coverage, presented by Bird et al. (2016), was based on the first 1000 orbits of INTEGRAL, i.e. the data acquired from the launch at the end of 2002 until the end of 2010. This catalog includes 939 sources detected above a significance threshold of $4.5 \sigma$ in the $17-100 \mathrm{keV}$ energy band on sky maps with different exposures.

In this work, we extend the INTEGRAL/IBIS all-sky survey to 17 years, using the whole set of observations carried out between 2002 December and 2020 January. We obtain a statistically clean catalog of hard X-ray sources detected above the $4.5 \sigma$ threshold along with their classification, if available. This list includes 46 newly detected sources. We also provide a sample of sub-threshold, $4.0-$ $4.5 \sigma$, INTEGRAL sources that were already known from previous INTEGRAL and Swift hard X-ray catalogs. In total, the final catalog comprises 929 hard X-ray sources. 


\section{DATA ANALYSIS}

For the current hard X-ray survey, we utilized all publicly available INTEGRAL data acquired by the ISGRI low-energy detector layer (Lebrun et al. 2003) of the IBIS telescope between 2002 December and 2020 January (spacecraft revolutions 26-2180). This corresponds to a total nominal exposure time of $406 \mathrm{Ms}$. We reduced the IBIS/ISGRI data with the INTEGRAL data analysis software developed at $\mathrm{IKI}^{1}$ (see e.g., Krivonos et al. 2010a, 2012; Churazov et al. 2014, and references therein). Below we describe details of the data analysis that are relevant for the current work.

We first applied the latest energy calibration (Caballero et al. 2013) for the registered IBIS/ISGRI detector events with the INTEGRAL Offline Scientific Analysis (OSA) provided by the INTEGRAL Science Data Centre (ISDC) Data Centre for Astrophysics up to the COR level. Because the latest OSA version 11.0 is only applicable to the data obtained since 26 December 2015 (revolution 1626), we calibrated ISGRI events obtained within the orbit ranges of 26-1625 and 1626-2180 with OSA versions 10.2 and 11.0, respectively,

We then produced a sky image of every individual INTEGRAL observation with a typical exposure time of $2 \mathrm{ks}$ (referred as a Science Window, or $S c W$ ). The flux scale in each $S c W$ sky image was adjusted to the flux of the Crab nebula measured in the nearest observation. This procedure was applied to account for the loss of sensitivity at low energies $E \lesssim 25 \mathrm{keV}$ caused by ongoing detector degradation.

\subsection{Energy bands}

To be consistent with our previous works, we chose $17-60 \mathrm{keV}$ as a working energy band for detection of sources in the present hard $\mathrm{X}$-ray survey. This also allows us to utilize the time period of spacecraft orbits before $\sim 1000$ when ISGRI had good sensitivity in the $\sim 17-30 \mathrm{keV}$ band. To determine an energy range that is not affected by detector aging, we analyzed the long-term (2003-2019) light curve of the persistent X-ray source Ophiuchus cluster in different energy bands and found that at energies above $30 \mathrm{keV}$ ISGRI demonstrates stable efficiency over the whole period of observations (Kuznetsova et al. 2022). We selected $30-80 \mathrm{keV}$ as an additional energy range for studying source light curves. Finally, in order to provide rough broad-band spectral information, we analysed the sky in logarithmically spaced energy bands of 17-26, 26-38, 38-57, 57-86, 86-129, 129-194 and 194-290 keV. Table 1 contains the list of the used energy bands and conversion factors between physical and mCrab units assuming the Crab spectrum in the form of $10(E / 1 \mathrm{keV})^{-2.1}$ photons $\mathrm{cm}^{-2} \mathrm{~s}^{-1} \mathrm{keV}^{-1}$.

\subsection{Sky map mosaics}

After applying selection criteria over the list of reconstructed $\mathrm{ScW}$ sky images, as described in Krivonos et al. (2007b), we obtained $157,200 \mathrm{ScWs}$ in each energy band, which comprises $273 \mathrm{Ms}$ of dead-time corrected exposure. Finally, we projected the sky images of all $S c W s$ on to $25^{\circ} \times 25^{\circ}$ sky frames covering the whole sky in the HEALPIX reference grid (Górski et al. 2005), with 192 frames in total.

The procedure of sky reconstruction for IBIS/ISGRI suffers from systematic noise, which is mainly caused by the presence of bright sources in the field of view (FOV) (see e.g., Krivonos et al. 2007c,

\footnotetext{
1 Space Research Institute of the Russian Academy of Sciences, Moscow,
} Russia
Table 1. Working energy bands for the current INTEGRAL all-sky survey.

\begin{tabular}{ccccc}
\hline \hline Name & $\begin{array}{c}\text { Range } \\
{[\mathrm{keV}]}\end{array}$ & $\begin{array}{c}\text { Width } \\
{[\mathrm{keV}]}\end{array}$ & \multicolumn{2}{c}{$1 \mathrm{mCrab}$} \\
{$\left[\mathrm{erg} \mathrm{s}^{-1} \mathrm{~cm}^{-2}\right]$} & {$\left[\mathrm{ph} \mathrm{cm}^{-2} \mathrm{~s}^{-1}\right]$} \\
\hline E1 & $17-60$ & 43 & $1.43 \times 10^{-11}$ & $3.02 \times 10^{-4}$ \\
E2 & $30-80$ & 50 & $1.06 \times 10^{-11}$ & $1.42 \times 10^{-4}$ \\
E3 & $17-26$ & 9 & $5.02 \times 10^{-12}$ & $1.50 \times 10^{-4}$ \\
E4 & $26-38$ & 12 & $4.31 \times 10^{-12}$ & $8.61 \times 10^{-5}$ \\
E5 & $38-57$ & 19 & $4.42 \times 10^{-12}$ & $5.98 \times 10^{-5}$ \\
E6 & $57-86$ & 29 & $4.31 \times 10^{-12}$ & $3.87 \times 10^{-5}$ \\
E7 & $86-129$ & 43 & $4.08 \times 10^{-12}$ & $2.43 \times 10^{-5}$ \\
E8 & $129-194$ & 65 & $3.94 \times 10^{-12}$ & $1.57 \times 10^{-5}$ \\
E9 & $194-290$ & 96 & $3.73 \times 10^{-12}$ & $9.89 \times 10^{-6}$ \\
\hline
\end{tabular}

2012). As a result, some sky artefacts are still present around persistent bright sources, such as Crab, Sco X-1, Cyg X-1, Cyg X-3, Vela X-1, GX 301-2 and GRS 1915+105, which requires manual inspection of the list of source candidates. Apart from the bright persistent sources, sky regions can be seriously contaminated by bright Galactic transients, which, however, can be excluded from the analysis. To produce sky maps that are as clean as possible, we avoided observations with angular offsets closer than $20^{\circ}$ from the following bright transients: IGR J17480-2446 (1519 - 1530, Bordas et al. 2010), GRS 1716-249 (1780 - 1806, Bassi et al. 2019), MAXI J1535-571 (1860 - 1865, Russell et al. 2019; Parikh et al. 2019), Swift J174510.8-262411 (1212 - 1264, Del Santo et al. 2016), MAXI J1820+070 (1931 - 1952, Shidatsu et al. 2018) and MAXI J1348-630 (2050 - 2061, Yatabe et al. 2019), where the range in parenthesis means the interval of excluded spacecraft orbits.

\subsection{Detection of sources}

Sources were searched as excesses on $25^{\circ} \times 25^{\circ}$ ISGRI sky frames, convolved with a Gaussian with $\sigma=5^{\prime}$, which approximates the effective point spread function (PSF) of IBIS/ISGRI. The signal-tonoise $(\mathrm{S} / \mathrm{N})$ distribution of pixels is dominated by the statistical noise and can be described by a Gaussian with zero mean and unit variance, as demonstrated in our previous works (e.g., Krivonos et al. 2007b). However, in sky regions that contain very bright point sources or the crowded field near the Galactic center, the root-mean-square (RMS) scatter of the $\mathrm{S} / \mathrm{N}$ distribution increases (e.g., Krivonos et al. 2010a), which requires manual inspection of detected excesses, as was already noted above.

We considered two criteria for detecting sources. First, a source is considered to be detected if the significance of its detection exceeds $4.5 \sigma$. Given the IBIS/ISGRI angular resolution of $12^{\prime}$, the all-sky map may be regarded as consisting of $\sim 10^{6}$ statistically independent pixels, which implies that less than 10 false detections are expected to be registered due to statistical fluctuations. The second, alternative, criterion, is intended to increase the completeness of the survey with respect to previously known hard X-ray sources that fall slightly below the $4.5 \sigma$ detection threshold. Specifically, if the spatial position of an INTEGRAL source candidate detected with a significance of $4.0-4.5 \sigma$ is consistent with that of a known hard X-ray source from an external catalog it is considered to be detected. To this end, we cross-correlated the list of $S / N>4.0 \sigma$ source candidates with previous INTEGRAL/IBIS and Swift/BAT hard X-ray source catalogs that are listed in Table 2. The search was done within a radius of 


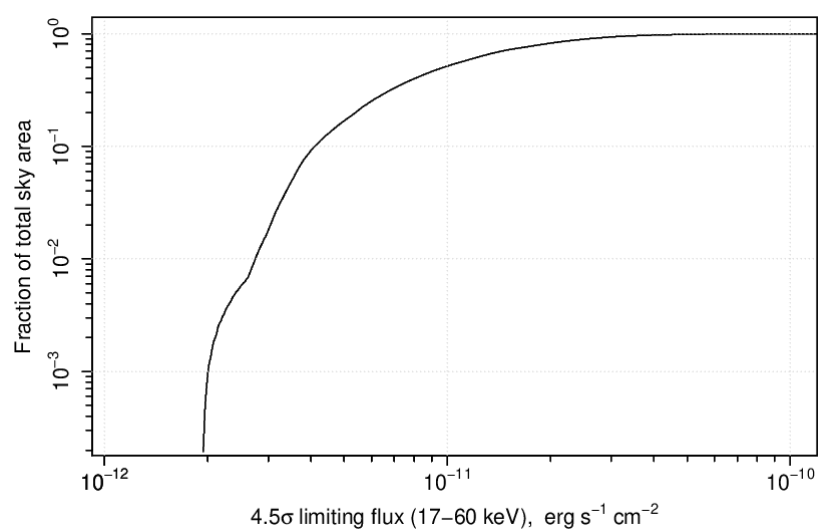

Figure 1. Fraction of the total sky area surveyed as a function of the limiting flux for source detection with $4.5 \sigma$ significance in the $17-60 \mathrm{keV}$ energy band.

$10^{\prime}$. Table 2 also provides the number of cross-matches with a given catalog.

As a result, all $4.0<S / N<4.5$ sources included in the final catalog are known hard X-ray sources. Consequently, we mark $S / N>$ $4.5 \sigma$ sources that do not have a counterpart in any of the hard X-ray surveys listed in Table 2 as newly detected in hard X-rays, except for a number of known X-ray transients and historical X-ray sources that are not listed in the mentioned hard X-ray catalogs for some reason. There are in total 46 newly detected hard X-ray sources.

\subsection{Survey sensitivity}

Figure 1 shows the survey sky coverage as a function of the flux corresponding to the $4.5 \sigma$ significance limit. The peak sensitivity, reached in the Galactic Center region, is about 0.14 mCrab $(2 \times$ $10^{-12} \mathrm{erg} \mathrm{s}^{-1} \mathrm{~cm}^{-2}$ ) in the $17-60 \mathrm{keV}$ energy band. Compared to our previous work based on 14 years of data (Krivonos et al. 2017) the peak sensitivity in this band has improved by nearly $10 \%$. The present survey covers $10 \%$ of the sky down to a flux limit of $\sim 0.3 \mathrm{mCrab}\left(4.3 \times 10^{-12} \mathrm{erg} \mathrm{s}^{-1} \mathrm{~cm}^{-2}\right)$ and $90 \%$ of the sky down to $\sim 1.8 \mathrm{mCrab}\left(2.6 \times 10^{-11} \mathrm{erg} \mathrm{s}^{-1} \mathrm{~cm}^{-2}\right)$ in the $17-60 \mathrm{keV}$ energy band. A limiting $(4.5 \sigma)$ flux of $1 \mathrm{mCrab}$ or better is achieved for $\sim 70 \%$ of the sky.

\section{CATALOG OF SOURCES}

The catalog of detected sources was compiled in the reference 17 $60 \mathrm{keV}$ energy band following the detection criteria described in Section 2.3. In addition, Table 3 provides source counts statistics for all energy bands considered in this work. The list of sources detected in the $17-60 \mathrm{keV}$ band is presented in Table A1 in Appendix A, and its content is described below.

Column (1) "Id" - sequence number of the source in the current catalog.

Column (2) "Name" - name of the source. Common names are given for sources which were known before their detection with $I N$ TEGRAL. Sources discovered by INTEGRAL or those whose nature was established thanks to the INTEGRAL observations are named as "IGR".

Columns (3,4) “RA, Dec" - source equatorial (J2000) coordinates. The positional accuracy depends on the significance of detection by IBIS/ISGRI (Gros et al. 2003; Bird et al. 2007). According to Krivonos et al. (2007b), the estimated $68 \%$ confidence intervals for sources detected at $5-6,10$, and $>20 \sigma$ are $2.1^{\prime}, 1.5^{\prime}$, and $<0.8^{\prime}$, respectively.

Column (5) "Flux" - time-averaged flux of the source in the 17-60 keV energy band. The online version of the table also contains source fluxes in all energy bands listed in Table 1.

Column (6) "Type" - general astrophysical type of the object. LMXB (HMXB) - low- (high-) mass X-ray binary; X-RAY BINARY - X-ray binary of uncertain type; CV - cataclysmic variable or symbiotic binary; SNR - supernova remnant; SNR/Pulsar - supernova remnant with a central pulsar (when both may contribute to the hard X-ray emission); MAGNETAR - magnetar (anomalous X-ray pulsars and soft gamma-ray repeaters); SUPERNOVA - supernova; STAR - active star (of various types, excluding the previously listed types of stellar objects); ULX - ultraluminous X-ray source; SEYFERT AGN of the Seyfert or LINER type; BLAZAR - beamed AGN (BL Lac objects and flat-spectrum radio quasars); AGN - unclassified AGN (i.e., the object is known to be an AGN in a general sense but detailed optical classification is missing); CLUSTER - a cluster of galaxies; and UNIDENT - an unclassified object. A question mark indicates that the specified type is not firmly established. These classifications are mainly based on the information contained in the $\mathrm{Simbad}^{2}$ and $\mathrm{NED}^{3}$ databases as well as in the previous versions of the INTEGRAL/IBIS and Swift/BAT hard X-ray source catalogs (see Table 2). In addition, we strove to select the most recent and reliable source identifications from the literature if necessary.

Column (7) "Notes" - additional notes such as references, redshift information, alternative source names, spatial confusion and transient flags. The references are mainly provided for recently discovered sources and are related to determination of their nature. The redshifts of the extragalactic sources were adopted from SIMBAD, NED and the Swift/BAT AGN Spectroscopic Survey (BASS) (see Ricci et al. 2017, and references therein).

\section{DISCUSSION AND SUMMARY}

In this work we present the all-sky survey of hard X-ray sources in the reference $17-60 \mathrm{keV}$ energy band, based on the data archive of the IBIS coded-mask telescope on board the INTEGRAL observatory accumulated over 17 years, from 2002 December to 2020 January. The combined catalog of sources includes 929 objects, 890 of which exceed a detection threshold of $4.5 \sigma$ and the rest are known hard X-ray sources detected at $4.0 \sigma<S / N<4.5 \sigma$.

\subsection{Properties of the catalog}

Table 4 presents source statistics by astrophysical type and Table 5 lists source counts over three categories: Galactic objects, sources located in the Large and Small Magellanic Clouds, and extragalactic objects. 113 sources from the catalog remain unclassified.

We mark 46 sources detected at $S / N>4.5 \sigma$ as newly detected in hard X-rays since they do not have a counterpart in any of the selected hard X-ray catalogs (Table 2). We cross-correlated these sources with a number of catalogs in the soft X-ray band, namely the ROSAT

\footnotetext{
2 Simbad Astronomical Database http://simbad.u-strasbg. fr

3 NASA/IPAC Extragalactic Database http://ned.ipac. caltech.edu
} 
Table 2. List of selected hard X-ray catalogs used for cross-matching.

\begin{tabular}{ccclr}
\hline \hline X-ray survey & Band (keV) & Sources & Cross-matches ${ }^{\mathrm{a}}$ & Reference \\
\hline \multicolumn{5}{c}{ Swift/BAT } \\
\hline all-sky, 70 months & $14-195$ & 1210 & $617(+20)$ & Baumgartner et al. (2013) \\
all-sky, 105 months & $14-195$ & 1632 & $693(+18)$ & Oh et al. (2018) \\
\hline \multicolumn{5}{c}{ INTEGRAL/IBIS } \\
\hline 1st catalog & $20-100$ & 123 & $122(+6)$ & Bird et al. (2004) \\
$\sim 50 \%$ of sky, 2nd cat. & $20-100$ & 209 & $197(+8)$ & Bird et al. (2006) \\
$\sim 70 \%$ of sky, 3rd cat. & $17-100$ & 421 & $358(+12)$ & Bird et al. (2007) \\
all-sky, 4th catalog & $17-100$ & 723 & $485(+12)$ & Bird et al. (2016) \\
all-sky, 1000 orbits & $17-100$ & 939 & $629(+14)$ & Krivonos et al. (2007b) \\
all-sky 3.5 years & $17-60$ & 403 & $369(+13)$ & Krivonos et al. (2010b) \\
all-sky, 7 years & $17-60$ & 521 & $486(+15)$ & Krivonos et al. (2012) \\
$|b|<17.5^{\circ}, 9$ years & $17-80$ & 402 & $387(+13)$ & Krivonos et al. (2017) \\
$|b|<17.5^{\circ}, 14$ years & $17-60$ & 72 & $70(+2)$ & Mereminskiy et al. (2016) \\
deep extragal. fields & $17-60$ & 147 & $140(+4)$ & \\
\hline
\end{tabular}

${ }^{a}$ The result of cross-correlation is regarded as positive if at least one source from the INTEGRAL/IBIS 17-year catalog is found within $10^{\prime}$ from a source in the external catalog. The number of cases where there is more than one INTEGRAL source within $10^{\prime}$ from the same source in the external catalog is given in brackets.

Table 3. Source detection statistics in different energy bands (see Table 1 for reference) of the current INTEGRAL/IBIS 17-year all-sky survey.

\begin{tabular}{cccc}
\hline \hline Band & S/N > 4.5 & $4.0>\mathrm{S} / \mathrm{N}>4.5^{\text {a }}$ & Total \\
\hline E1 & 890 & 39 & 929 \\
E2 & 693 & 40 & 733 \\
E3 & 554 & 45 & 599 \\
E4 & 700 & 55 & 755 \\
E5 & 597 & 48 & 645 \\
E6 & 314 & 33 & 347 \\
E7 & 208 & 29 & 237 \\
E8 & 63 & 9 & 72 \\
E9 & 17 & 5 & 22 \\
\hline
\end{tabular}

${ }^{a}$ Such sources are required to have been previously detected in any of the all-sky hard X-ray surveys listed in Table 2.

all-sky bright source catalog (1RXS; Voges et al. 1999), the XMMNewton slew survey (XMMSL2; Saxton et al. 2008), the XMMNewton serendipitous survey (Watson et al. 2009; Webb et al. 2020), the Swift/XRT point-source catalog (2SXPS; Evans et al. 2020), and the Chandra source catalog 2.0 (Evans et al. 2010). Specifically, we searched for soft X-ray counterparts within $5^{\prime}$ of the positions of the INTEGRAL sources and only considered sources with a significant detection above $2 \mathrm{keV}$ and the corresponding X-ray flux $F_{X} \gtrsim 10^{-13}$ erg s${ }^{-1} \mathrm{~cm}^{-2}$. We then searched for a positionally coincident optical or radio source using the VizieR (Ochsenbein et al. 2000) service, in order to determine the source class. As a result, we have found soft X-ray counterparts for 8 out of the 46 new INTEGRAL sources and classified some of these objects (see Table 6). Most of them are likely of extragalactic nature.

The identification completeness of the survey, i.e. the fraction of identified sources, is $\left(N_{\mathrm{Tot}}-N_{\mathrm{NotID}}\right) / N_{\mathrm{Tot}}=1-113 / 929 \sim 0.88$. Interestingly, it is the same for both the Galactic $\left(|b|<5^{\circ}\right)$ and
Table 4. Statistics of sources in the catalog.

\begin{tabular}{cl}
\hline \hline Type & Count (Notes) \\
\hline LMXB & 145 \\
HMXB & 115 \\
X-ray binary (unclassified) & 1 (SWIFT J1858.6-0814) \\
CV & 79 \\
Star & 4 \\
Magnetar & 5 \\
SNR, SNR/Pulsar & 25 \\
Molecular cloud & 1 (Sgr B2) \\
Galactic Center & 1 (Sgr A*) \\
Supernova & $1($ AT2018cow) \\
ULX & 2 \\
Seyfert galaxy & 336 \\
AGN (unclassified) & 39 \\
Blazar & 54 \\
Galaxy cluster & 8 \\
Unidentified & 113 \\
\hline
\end{tabular}

Table 5. Statistics of identified sources by category.

\begin{tabular}{cl}
\hline Category & Count \\
\hline Galactic & 356 \\
Magellanic Clouds & 20 \\
Extragalactic & 440 \\
\hline
\end{tabular}

extragalactic $\left(|b|>5^{\circ}\right)$ parts of the sky. As expected, due to the continuously increasing depth of the INTEGRAL all-sky survey, the fraction of identified sources sources has decreased compared to our previous compilation of the all-sky catalog, where it was $93 \%$ and $96 \%$ at $|b|<5^{\circ}$ and $|b|>5^{\circ}$, respectively (Krivonos et al. 2010b).

Most of the INTEGRAL sources in the current survey have X-ray counterparts in the Swift/BAT 70- and 105-month catalogs (Baum- 
Table 6. Soft X-ray $(E>2 \mathrm{keV})$ counterparts of INTEGRAL sources discovered in the hard X-ray $(17-60 \mathrm{keV})$ band.

\begin{tabular}{clccl}
\hline \hline Name & X-ray & Offset & Type & Notes \\
\hline IGR J04085-6546 & 2RXS J040840.0-654545, XMMSL2 J040839.1-654600 & $0.3^{\prime}$ & AGN & LEDA 310383, z=0.125 (Spiro et al. 2013) \\
IGR J07328-4640 & 2RXS J073245.8-464006, 4XMM J073244.3-464017 & $1.4^{\prime}$ & BLAZAR? & PKS 0731-465 \\
IGR J10595-5125 & 2RXS J105920.3-512644, 2SXPS J105918.9-512632 & $2.3^{\prime}$ & SEYFERT & ESO 215-14, z=0.019 \\
IGR J16005-4645 & 2RXS J160019.6-464802, 2SXPS J160020.4-464841 & $3.6^{\prime}$ & AGN? & Edelson \& Malkan (2012) \\
IGR J17227+3411 & 4XMM J172230.8+341339, 2SXPS J172230.9+341341 & $3.5^{\prime}$ & AGN? & z=0.425 (Caccianiga et al. 2008) \\
IGR J17342-4049 & XMMSL2 J173425.6-405121 & $2.2^{\prime}$ & & \\
IGR J17449-3037 & 4XMM J174507.9-303905 & $3.4^{\prime}$ & & \\
IGR J18006-3426 & 2SXPS J180050.6-342322 & $4.1^{\prime}$ & & \\
\hline
\end{tabular}

gartner et al. 2013; Oh et al. 2018, respectively). Taking the different sky coverage by INTEGRAL/IBIS and Swift/BAT into account, it is interesting to investigate the properties of the INTEGRAL sources that are not present in the Swift/BAT catalogs. According to Table 2, 711 INTEGRAL sources have counterparts in the Swift/BAT 105-month survey and 218 (929-711) sources are missed. The majority of the unmatched INTEGRAL sources (165 out of 218) prove to be located near the Galactic plane at $|b|<17.5^{\circ}$, which is the characteristic latitude span of the INTEGRAL Galactic surveys (Krivonos et al. 2012). This indicates that the INTEGRAL/IBIS survey has higher sensitivity near the Galactic plane compared to the Swift/BAT surveys. Similar evidence has been demonstrated by Bird et al. (2016) in comparing their INTEGRAL catalog with the Swift/BAT 70-month survey by Baumgartner et al. (2013). Among the 53 INTEGRAL sources at $|b|>17.5^{\circ}$ that are absent in the Swift/BAT 105-month catalog there are 5 HMXBs, 11 non-blazar AGN, 4 blazars, 32 unidentified objects, and the supernova AT2018cow; 17 are newly discovered sources in hard X-ray domain (see Sect. 2.3).

Bird et al. (2016) (hereafter B16) presented a hard X-ray source catalog based on INTEGRAL/IBIS observations performed in the first 1000 orbits of INTEGRAL (up to the end of 2010). This catalog includes 939 sources detected in the $17-100 \mathrm{keV}$ band above a $4.5 \sigma$ significance threshold. The final catalog was constructed using the "burstcity" method, based on finding the time window wherein the significance of detection of a given source is highest. As a result, 342 sources (out of 939) are transients found on different timescales, labeled with a variability flag in the B16 catalog. We have crosscorrelated our source list with the B16 catalog and found 643 matches within a 10 ' radius. Among them, 140 sources are marked with a ' $\mathrm{Y}$ ' flag by B16, which implies moderate variability, and 12 are strongly variable sources, labeled as 'YY'. The remaining 491 sources are not labeled as variable in the B16 catalog. Therefore, the majority $(76 \%)$ of our 643 sources that have matches in the B16 catalog are presumed to be persistent ones, whereas the $\sim 300$ B16 sources that are not confirmed by the present survey are mainly transients detected on INTEGRAL/IBIS sky maps of various timescales and not present on the all-time maps (see B16 for details). However, $~ 100$ persistent B16 sources have not been found in our catalog either. These sources are characterized by sub-mCrab fluxes and have a median detection significance $\sim 6 \sigma$, i.e. they were found close to the detection threshold in the B16 survey. Possible explanations for their absence in the present catalog may lie in the different data analysis methods and slightly different energy bands, substantial source variability, and some of the weak B16 sources being false detections. Because B16 is the most recent previous INTEGRAL/IBIS all-sky survey, we added a special flag for all 286 new sources in our catalog that were not detected by $\mathrm{B} 16$.

\subsection{Extragalactic $\log N-\operatorname{LogS}$}

Assuming that AGNs are uniformly distributed over the sky, we can construct their number-flux function in the hard X-ray band. As INTEGRAL observations cover the sky inhomogeneously, we must take the sensitivity map into account. To this end, we divided the observed source counts by the sky coverage at the $4.5 \sigma$ level as a function of flux (Fig. 1). Figure 2 shows the resulting cumulative $\log N$-Log $S$ distribution based on the 356 non-blazar AGNs detected at $S / N>4.5 \sigma$ over the whole sky. AGN $\log N-\log S$ distributions are usually approximated by a power law $N(>S)=A S^{-\alpha}$. Using a maximum-likelihood estimator (Jauncey 1967; Crawford et al. 1970), we determined the best-fit value of the slope $\alpha=1.44 \pm 0.09$ using the source counts at fluxes above $10^{-11} \mathrm{erg} \mathrm{s}^{-1} \mathrm{~cm}^{-2}$, where our source sample is expected to be highly complete. We fixed the normalization of the power law at the observed value of the $\log N-\log S$ distribution at $S=10^{-11} \mathrm{erg} \mathrm{s}^{-1} \mathrm{~cm}^{-2}, A=8 \times 10^{-3} \mathrm{deg}^{-2}$. The inferred $\log N-\log S$ slope $\alpha$ is consistent with a homogeneous distribution of sources in space $(\alpha=3 / 2)$.

At fluxes below $\sim 5 \times 10^{-12} \mathrm{erg} \mathrm{s}^{-1} \mathrm{~cm}^{-2}$, the measured $\log N$ $\log S$ demonstrates a significant deficit of observed source counts relative to the aforementioned power-law dependence. This occurs far above both the known flattening of AGN number counts (in the $2-10 \mathrm{keV}$ energy band) at $\sim 10^{-14} \mathrm{erg} \mathrm{s}^{-1} \mathrm{~cm}^{-2}$ (Georgakakis et al. 2008) and the upturn at fluxes below $\sim 2 \times 10^{-13} \mathrm{erg} \mathrm{s}^{-1} \mathrm{~cm}^{-2}$ observed between the NuSTAR and Swift/BAT number-flux relations (Ajello et al. 2012; Harrison et al. 2016; Akylas \& Georgantopoulos 2019, see also below). Thus, no deviations from the canonical $\alpha=$ $3 / 2$ are expected at fluxes $\lesssim 10^{-11} \mathrm{erg} \mathrm{s}^{-1} \mathrm{~cm}^{-2}$, and the observed slope flattening is likely caused by incompleteness of the source sample. To check this, we constructed an all-sky $\log N-\log S$ relation for the 104 unclassified sources at $S / N>4.5 \sigma$, which is shown in Fig. 2. This $\log N-\log S$ is well approximated with a power law and characterized by a steep $\alpha=2.8 \pm 0.2$ and normalization $A=$ $4.9 \times 10^{-3} \mathrm{deg}^{-2}$ at $S=5 \times 10^{-12} \mathrm{erg} \mathrm{s}^{-1} \mathrm{~cm}^{-2}$. It turns out that the combined $\log N-\log S$ distribution of unidentified sources and non-blazar AGNs follows a power law with $\alpha \sim 3 / 2(\alpha=1.40 \pm 0.05$; $A=8.7 \times 10^{-3} \mathrm{deg}^{-2}$ at $S=10^{-11} \mathrm{erg} \mathrm{s}^{-1} \mathrm{~cm}^{-2}$; calculated over the whole range of fluxes), as demonstrated in Fig. 2. This suggests that the majority of the unidentified sources are of extragalactic origin.

Over the past two decades, number counts of extragalactic objects in hard X-ray domain have been independently measured with INTEGRAL/IBIS (e.g., Krivonos et al. 2005, 2007b, 2015; Beck- 


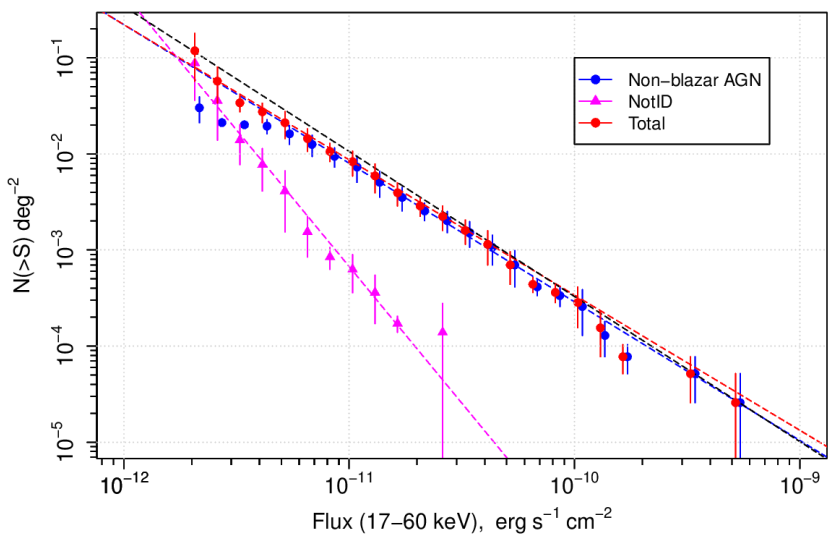

Figure 2. Binned number-flux relation for the 356 non-blazar AGNs (Seyferts and unclassified AGNs, blue points), 104 unidentified sources (magenta triangles), and 460 objects in total (red points) detected above the $4.5 \sigma$ detection level. The binning of the blue and red points is slightly shifted for better visibility. The best-fitting power laws for the non-blazar AGNs and unidentified sources are shown by the dashed lines in the corresponding colors. For comparison, the dashed black line shows the AGN number-flux relation from Akylas \& Georgantopoulos (2019) based on the Swift/BAT 105-months source catalog (Oh et al. 2018).

mann et al. 2006; Bazzano et al. 2006) and Swift/BAT (e.g., Tueller et al. 2008; Ajello et al. 2012). Krivonos et al. (2010c) compared the AGN $\log N-\log S$ distribution determined with INTEGRAL/IBIS in the $17-60 \mathrm{keV}$ band with the one derived in the $15-55 \mathrm{keV}$ band from the Swift/BAT AGN sample by Ajello et al. (2009) and found an excellent agreement (see also Cusumano et al. 2010). On the other hand, Harrison et al. (2016) presented NuSTAR 8-24 keV AGN number counts at lower fluxes that lay significantly above a simple extrapolation with a Euclidean slope of the Swift/BAT counts. However, Akylas \& Georgantopoulos (2019) demonstrated that the bright part of the NUSTAR AGN number counts was in agreement with the Swift/BAT counts.

In light of the updated INTEGRAL AGN number counts, we compare them in Fig. 2 with the Swift/BAT 105-month AGN $\log N-\log S$ modelled by Akylas \& Georgantopoulos (2019). To this end, we converted the 14-195 keV Swift/BAT number counts to the $17-60 \mathrm{keV}$ band following Krivonos et al. (2010c). As seen from Fig. 2, the Swift/BAT $\log N-\log S$ has a slope $(\alpha=1.51 \pm 0.10$, Akylas \& Georgantopoulos 2019), which is better consistent with the canonical $\alpha=3 / 2$ value than the slope obtained in the present work but is consistent with the latter as well. The small difference between Swift/BAT and INTEGRAL/IBIS may be partially caused by the fact that Akylas \& Georgantopoulos (2019) included beamed AGNs in their counts while we tried to select only non-blazar AGN (although some blazars may be present among our unclassified AGN and unidentified sources). Nevertheless, our $\log N-\log S$ tends to be even less compatible with the NuSTAR number counts than Swift/BAT.

Finally, we plan to maintain an extended online version of the INTEGRAL/IBIS 17-year hard X-ray source catalog at http: //integral . cosmos . ru where additional information will be presented. Specifically, we plan to provide soft X-ray and optical coordinates (where available), spectral information, and multi-year light curves of the detected sources, as well as sky images in different energy bands up to $290 \mathrm{keV}$.

\section{ACKNOWLEDGEMENTS}

We thank the anonymous referee for many suggestions that helped us to improve the paper. This work is based on observations with INTEGRAL, an ESA project with instruments and the science data centre funded by ESA member states (especially the PI countries: Denmark, France, Germany, Italy, Switzerland, Spain), and Poland, and with the participation of Russia and the USA. This research has made use of: data obtained from the High Energy Astrophysics Science Archive Research Center (HEASARC) provided by NASA's Goddard Space Flight Center; the SIMBAD database operated at CDS, Strasbourg, France; the NASA/IPAC Extragalactic Database (NED) operated by the Jet Propulsion Laboratory, California Institute of Technology, under contract with the National Aeronautics and Space Administration. The data were obtained from the European ${ }^{4}$ and Russian ${ }^{5}$ INTEGRAL Science Data Centers. The authors are grateful to E. M. Churazov, who developed the INTEGRAL/IBIS data analysis methods and provided the software, and thank the Max Planck Institute for Astrophysics for computational support. This work was financially supported by grant 19-12-00396 from the Russian Science Foundation.

\section{DATA AVAILABILITY}

This work is based on publicly available data of the INTEGRAL observatory. The ISDC Data Centre for Astrophysics (http://isdc. unige.ch/) provides the scientific archive of the INTEGRAL data to the community.

\section{REFERENCES}

Acero F., et al., 2015, ApJS, 218, 23

Aharonian F., et al., 2005, A\&A, 442, 1

Ajello M., et al., 2009, ApJ, 699, 603

Ajello M., Alexander D. M., Greiner J., Madejski G. M., Gehrels N., Burlon D., 2012, ApJ, 749, 21

Akylas A., Georgantopoulos I., 2019, A\&A, 625, A131

Altamirano D., et al., 2008, The Astronomer's Telegram, 1651, 1

Apparao K. M. V., Bignami G. F., Maraschi L., Helmken H., Margon B., Hjellming R., Bradt H. V., Dower R. G., 1978, Nature, 273, 450

Armas Padilla M., Muñoz-Darias T., Sánchez-Sierras J., De Marco B., Jiménez-Ibarra F., Casares J., Corral-Santana J. M., Torres M. A. P., 2019, MNRAS, 485, 5235

Bahramian A., Gladstone J. C., Heinke C. O., Wijnands R., Kaur R., Altamirano D., 2014, MNRAS, 441, 640

Barlow E. J., et al., 2005, A\&A, 437, L27

Barlow E. J., Knigge C., Bird A. J., J Dean A., Clark D. J., Hill A. B., Molina M., Sguera V., 2006, MNRAS, 372, 224

Barthelmy S. D., et al., 2005, Space Sci. Rev., 120, 143

Barthelmy S. D., et al., 2019, The Astronomer's Telegram, 12436, 1

Bassani L., et al., 2004, The Astronomer's Telegram, 232, 1

Bassani L., et al., 2005, ApJ, 634, L21

Bassani L., et al., 2006, ApJ, 636, L65

Bassani L., et al., 2007, ApJ, 669, L1

Bassani L., et al., 2009, MNRAS, 395, L1

Bassani L., Landi R., Malizia A., Stephen J. B., Bazzano A., Bird A. J., Ubertini P., 2014, A\&A, 561, A108

Bassi T., et al., 2019, MNRAS, 482, 1587

Baumgartner W. H., Tueller J., Markwardt C., Skinner G., 2010, in AAS/High Energy Astrophysics Division \#11. p. 13.05

\footnotetext{
${ }^{4}$ http://isdc.unige.ch

5 http://hea.iki.rssi.ru/rsdc
} 
Baumgartner W. H., Tueller J., Markwardt C. B., Skinner G. K., Barthelmy S., Mushotzky R. F., Evans P. A., Gehrels N., 2013, ApJS, 207, 19

Bazzano A., et al., 2006, ApJ, 649, L9

Beckmann V., et al., 2005, ApJ, 631, 506

Beckmann V., Soldi S., Shrader C. R., Gehrels N., Produit N., 2006, ApJ, 652,126

Bélanger G., et al., 2006, ApJ, 636, 275

Beri A., et al., 2021, MNRAS, 500, 565

Bernardini F., de Martino D., Falanga M., Mukai K., Matt G., Bonnet-Bidaud J. M., Masetti N., Mouchet M., 2012, A\&A, 542, A22

Bernardini F., de Martino D., Mukai K., Israel G., Falanga M., Ramsay G., Masetti N., 2015, MNRAS, 453, 3100

Bernardini F., de Martino D., Mukai K., Falanga M., 2018, MNRAS, 478, 1185

Bikmaev I. F., Sunyaev R. A., Revnivtsev M. G., Burenin R. A., 2006, Astronomy Letters, 32, 221

Bikmaev I. F., Burenin R. A., Revnivtsev M. G., Sazonov S. Y., Sunyaev R. A., Pavlinsky M. N., Sakhibullin N. A., 2008a, Astronomy Letters, 34, 653

Bikmaev I., Revnivtsev M., Burenin R., Sazonov S., Sunyaev R., Pavlinsky M., Galeev A., Sakhibullin N., 2008b, The Astronomer's Telegram, 1363, 1

Bikmaev I., et al., 2017, The Astronomer's Telegram, 10968, 1

Bird A. J., et al., 2004, ApJ, 607, L33

Bird A. J., et al., 2006, ApJ, 636, 765

Bird A. J., et al., 2007, ApJS, 170, 175

Bird A. J., et al., 2010, ApJS, 186, 1

Bird A. J., et al., 2016, ApJS, 223, 15

Bodaghee A., Walter R., Zurita Heras J. A., Bird A. J., Courvoisier T. J. L., Malizia A., Terrier R., Ubertini P., 2006, A\&A, 447, 1027

Bodaghee A., Tomsick J. A., Rodriguez J., 2012, ApJ, 753, 3

Bordas P., et al., 2010, The Astronomer's Telegram, 2919, 1

Bozzo E., Beardmore A., Papitto A., Ferrigno C., Gibaud L., 2011, The Astronomer's Telegram, 3558, 1

Bozzo E., Romano P., Falanga M., Ferrigno C., Papitto A., Krimm H. A., 2015, A\&A, 579, A56

Bozzo E., et al., 2018a, A\&A, 613, A22

Bozzo E., Savchenko V., Ferrigno C., Ducci L., Kuulkers E., Ubertini P., Laurent P., 2018b, The Astronomer's Telegram, 11478, 1

Brandt S., Budtz-Jørgensen C., Chenevez J., 2006a, The Astronomer's Telegram, 778, 1

Brandt S., Budtz-Jorgensen C., Chenevez J., Lund N., Oxborrow C. A., Westergaard N. J., 2006b, The Astronomer's Telegram, 970, 1

Brandt S., Budtz-Jørgensen C., Gotz D., Hurley K., Frontera F., 2007, The Astronomer's Telegram, 1054, 1

Brandt S., et al., 2008, The Astronomer's Telegram, 1400, 1

Britt C. T., et al., 2013, ApJ, 769, 120

Burenin R., Mescheryakov A., Revnivtsev M., Bikmaev I., Sunyaev R., 2006a, The Astronomer's Telegram, 880, 1

Burenin R., Mescheryakov A., Sazonov S., Revnivtsev M., Bikmaev I., Sunyaev R., 2006b, The Astronomer's Telegram, 883, 1

Burenin R., Revnivtsev M., Mescheryakov A., Bikmaev I., Pavlinsky M., Sunyaev R., 2007, The Astronomer's Telegram, 1270, 1

Burenin R. A., Mescheryakov A. V., Revnivtsev M. G., Sazonov S. Y., Bikmaev I. F., Pavlinsky M. N., Sunyaev R. A., 2008, Astronomy Letters, 34,367

Burenin R., Makarov D., Uklein R., Revnivtsev M., Lutovinov A., 2009, The Astronomer's Telegram, 2193, 1

Butler S. C., et al., 2009, ApJ, 698, 502

Caballero I., et al., 2013, arXiv e-prints, p. arXiv:1304.1349

Caccianiga A., et al., 2008, A\&A, 477, 735

Capitanio F., et al., 2006, ApJ, 643, 376

Chakrabarty D., Jonker P., Markwardt C. B., 2011a, The Astronomer's Telegram, 3218, 1

Chakrabarty D., Markwardt C. B., Linares M., Jonker P. G., 2011b, The Astronomer's Telegram, 3606, 1

Chaty S., Rahoui F., Foellmi C., Tomsick J. A., Rodriguez J., Walter R., 2008, A\&A, 484, 783
Chelovekov I. V., Grebenev S. A., 2007, Astronomy Letters, 33, 807

Chenevez J., et al., 2012, The Astronomer's Telegram, 4050, 1

Chernyakova M., Lutovinov A., Capitanio F., Lund N., Gehrels N., 2003, The Astronomer's Telegram, 157, 1

Chernyakova M., Lutovinov A., Rodríguez J., Revnivtsev M., 2005, MNRAS, 364,455

Churazov E., et al., 2007, A\&A, 467, 529

Churazov E., et al., 2014, Nature, 512, 406

Clavel M., et al., 2016, MNRAS, 461, 304

Clavel M., Tomsick J. A., Hare J., Krivonos R., Mori K., Stern D., 2019, ApJ, 887,32

Cocchi M., Bazzano A., Natalucci L., Ubertini P., Heise J., Muller J. M., in 't Zand J. J. M., 1999, A\&A, 346, L45

Coe M. J., Bartlett E. S., Bird A. J., Haberl F., Kennea J. A., McBride V. A., Townsend L. J., Udalski A., 2015, MNRAS, 447, 2387

Coleiro A., Chaty S., Zurita Heras J. A., Rahoui F., Tomsick J. A., 2013, A\&A, 560, A108

Cornelisse R., Verbunt F., in't Zand J. J. M., Kuulkers E., Heise J., 2002, A\&A, 392, 931

Courvoisier T. J. L., Walter R., Rodriguez J., Bouchet L., Lutovinov A. A., 2003, IAU Circ., 8063, 3

Crawford D. F., Jauncey D. L., Murdoch H. S., 1970, ApJ, 162, 405

Curran P. A., Chaty S., Zurita Heras J. A., 2011, A\&A, 533, A3

Cusumano G., et al., 2010, A\&A, 510, A48

Cusumano G., D’Aì A., Segreto A., La Parola V., Del Santo M., 2020, MNRAS, 498, 2750

D’Aì A., La Parola V., Cusumano G., Segreto A., Romano P., Vercellone S., Robba N. R., 2011, A\&A, 529, A30

Degenaar N., et al., 2007, The Astronomer's Telegram, 1136, 1

Degenaar N., et al., 2010, MNRAS, 404, 1591

Degenaar N., Yang Y. J., Wijnands R., 2011, The Astronomer's Telegram, 3741,1

Degenaar N., Altamirano D., Wijnands R., 2012, The Astronomer's Telegram, 4219,1

Del Monte E., et al., 2008a, in The 7th INTEGRAL Workshop. p. 122

Del Monte E., et al., 2008b, The Astronomer's Telegram, 1445, 1

Del Santo M., et al., 2011, The Astronomer's Telegram, 3210, 1

Del Santo M., Nucita A. A., Lodato G., Manni L., De Paolis F., Farihi J., De Cesare G., Segreto A., 2014, MNRAS, 444, 93

Del Santo M., et al., 2016, MNRAS, 456, 3585

Doroshenko V., et al., 2020a, MNRAS, 491, 1857

Doroshenko V., Tsygankov S., Long J., Santangelo A., Molkov S., Lutovinov A., Kong L. D., Zhang S., 2020b, A\&A, 634, A89

Ducci L., Kuulkers E., Grinberg V., Paizis A., Sidoli L., Bozzo E., Ferrigno C., Savchenko V., 2018, The Astronomer's Telegram, 11941, 1

Eckert D., Walter R., Kretschmar P., Mas-Hesse M., Palumbo G. G. C., Roques J. P., Ubertini P., Winkler C., 2004, The Astronomer's Telegram, 352,1

Eckert D., et al., 2013, The Astronomer's Telegram, 4925, 1

Edelson R., Malkan M., 2012, ApJ, 751, 52

Esposito P., Israel G. L., Sidoli L., Mason E., Rodríguez Castillo G. A., Halpern J. P., Moretti A., Götz D., 2013, MNRAS, 433, 2028

Evans I. N., et al., 2010, ApJS, 189, 37

Evans P. A., et al., 2020, ApJS, 247, 54

Falanga M., Bozzo E., Walter R., Sarty G. E., Stella L., 2011, Journal of the American Association of Variable Star Observers (JAAVSO), 39, 110

Ferrigno C., Bozzo E., Belloni L. G. A. P. T. M., 2011, The Astronomer's Telegram, 3560, 1

Ferrigno C., Bozzo E., Del Santo M., Capitanio F., 2012, A\&A, 537, L7

Fiocchi M., Bassani L., Bazzano A., Ubertini P., Landi R., Capitanio F., Bird A. J., 2010, ApJ, 720, 987

Fornasini F. M., Tomsick J. A., Bachetti M., Krivonos R. A., Fürst F., Natalucci L., Pottschmidt K., Wilms J., 2017, ApJ, 841, 35

Fortin F., Chaty S., Coleiro A., Tomsick J. A., Nitschelm C. H. R., 2018, A\&A, 618, A150

Fuerst F., et al., 2018, The Astronomer's Telegram, 11357, 1

Funk S., et al., 2007, A\&A, 470, 249

Gänsicke B. T., et al., 2005, MNRAS, 361, 141 
Gehrels N., et al., 2004, ApJ, 611, 1005

Georgakakis A., Nandra K., Laird E. S., Aird J., Trichas M., 2008, MNRAS, 388,1205

Gianní S., de Rosa A., Bassani L., Bazzano A., Dean T., Ubertini P., 2011, MNRAS, 411, 2137

Gibaud L., et al., 2011, The Astronomer's Telegram, 3565, 1

Goncalves T. S., Martin D. C., Halpern J. P., Eracleous M., Pavlov G. G., 2008, The Astronomer's Telegram, 1623, 1

González-Martín O., et al., 2011, A\&A, 527, A142

Goossens M. E., Bird A. J., Hill A. B., Sguera V., Drave S. P., 2019, MNRAS, 485,286

Górski K. M., Hivon E., Banday A. J., Wandelt B. D., Hansen F. K., Reinecke M., Bartelmann M., 2005, ApJ, 622, 759

Grebenev S. A., Sunyaev R. A., 2004, The Astronomer's Telegram, 342, 1

Grebenev S. A., Sunyaev R. A., 2007, Astronomy Letters, 33, 149

Grebenev S. A., Sunyaev R. A., 2010, Astronomy Letters, 36, 533

Grebenev S. A., Ubertini P., Chenevez J., Orr A., Sunyaev R. A., 2004, The Astronomer's Telegram, 275, 1

Grebenev S. A., Molkov S. V., Sunyaev R. A., 2005a, The Astronomer's Telegram, 444, 1

Grebenev S. A., Bird A. J., Molkov S. V., Soldi S., Kretschmar P., Diehl R., Budz-Joergensen C., McBreen B., 2005b, The Astronomer's Telegram, 457,1

Grebenev S. A., Molkov S. V., Sunyaev R. A., 2005c, The Astronomer's Telegram, 467, 1

Grebenev S. A., Molkov S. V., Revnivtsev M. G., Sunyaev R. A., 2007a, in The Obscured Universe. Proceedings of the VI INTEGRAL Workshop. p. 3730376 (arXiv: 0709.2313)

Grebenev S. A., Revnivtsev M. G., Sunyaev R. A., 2007b, The Astronomer's Telegram, 1319,

Grebenev S. A., Lutovinov A. A., Tsygankov S. S., Mereminskiy I. A., 2013, MNRAS, 428, 50

Grebenev S. A., Mereminskiy I. A., Prosvetov A. V., Ducci L., Bozzo E., Savchenko V., Ferrigno C., 2018, The Astronomer's Telegram, 11306, 1

Grebenev S. A., Mereminsky I. A., Bozzo E., Ferrigno C., Savchenko V., Ducci L., 2019, The Astronomer's Telegram, 13155, 1

Greiss S., Steeghs D., Maccarone T., Jonker P. G., Torres M. A. P., Gonzalez O., Masetti N., Rojas A., 2011, The Astronomer's Telegram, 3562, 1

Gros A., Goldwurm A., Cadolle-Bel M., Goldoni P., Rodriguez J., Foschini L., Del Santo M., Blay P., 2003, A\&A, 411, L179

Halpern J. P., 2006, The Astronomer's Telegram, 847, 1

Halpern J. P., Gotthelf E. V., 2008, The Astronomer's Telegram, 1457, 1

Halpern J. P., Thorstensen J. R., 2018, The Astronomer's Telegram, 11787, 1

Halpern J. P., Tyagi S., 2005, The Astronomer's Telegram, 681, 1

Halpern J. P., Thorstensen J. R., Cho P., Collver G., Motsoaledi M., Breytenbach H., Buckley D. A. H., Woudt P. A., 2018, AJ, 155, 247

Hannikainen D. C., Rodriguez J., Pottschmidt K., 2003, IAU Circ., 8088, 4

Hare J., Halpern J. P., Clavel M., Grindlay J. E., Rahoui F., Tomsick J. A., 2019, ApJ, 878, 15

Hare J., Halpern J. P., Tomsick J. A., Thorstensen J. R., Bodaghee A., Clavel M., Krivonos R., Mori K., 2021, ApJ, 914, 85

Harrison F. A., et al., 2016, ApJ, 831, 185

Heinke C. O., Tomsick J. A., Yusef-Zadeh F., Grindlay J. E., 2009, ApJ, 701, 1627

Heinke C. O., et al., 2019, The Astronomer's Telegram, 12843, 1

Hiemstra B., Méndez M., Done C., Díaz Trigo M., Altamirano D., Casella P., 2011, MNRAS, 411, 137

Huchra J. P., et al., 2012, ApJS, 199, 26

Jauncey D. L., 1967, Nature, 216, 877

Kaaret P., Morgan E. H., Vanderspek R., Tomsick J. A., 2006, ApJ, 638, 963

Kalamkar M., Homan J., Altamirano D., van der Klis M., Casella P., Linares M., 2011, ApJ, 731, L2

Kalemci E., Tomsick J. A., Rothschild R. E., Pottschmidt K., Corbel S., Kaaret P., 2006, ApJ, 639, 340

Karasev D. I., Lutovinov A. A., Grebenev S. A., 2007, Astronomy Letters, 33, 159

Karasev D. I., Lutovinov A. A., Burenin R. A., 2008, Astronomy Letters, 34, 753
Karasev D. I., Lutovinov A. A., Burenin R. A., 2010, MNRAS, 409, L69

Karasev D. I., Lutovinov A. A., Revnivtsev M. G., Krivonos R. A., 2012, Astronomy Letters, 38, 629

Karasev D. I., et al., 2018, Astronomy Letters, 44, 522

Karasev D. I., et al., 2020, Astronomy Letters, 45, 836

Kaur R., Wijnands R., Paul B., Patruno A., Degenaar N., 2010, MNRAS, 402, 2388

Kaur R., Kotulla R., Degenaar N., Wijnands R., Kaplan D., 2011, The Astronomer's Telegram, 3268, 1

Kaur R., Wijnands R., Kamble A., Cackett E. M., Kutulla R., Kaplan D., Degenaar N., 2017, MNRAS, 464, 170

Keek S., Kuiper L., Hermsen W., 2006, The Astronomer's Telegram, 810, 1

Kennea J. A., et al., 2019, The Astronomer's Telegram, 13195, 1

Kniazev A., Revnivtsev M., Burenin R., Tkachenko A., 2010, The Astronomer's Telegram, 2457, 1

Koss M., Mushotzky R., Veilleux S., Winter L. M., Baumgartner W., Tueller J., Gehrels N., Valencic L., 2011, ApJ, 739, 57

Koss M., et al., 2017, ApJ, 850, 74

Kretschmar P., Mereghetti S., Hermsen W., Ubertini P., Winkler C., Brandt S., Diehl R., 2004, The Astronomer's Telegram, 345, 1

Kretschmar P., et al., 2019, New Astron. Rev., 86, 101546

Krimm H. A., Tomsick J. A., Markwardt C. B., Brocksopp C., Grisé F., Kaaret P., Romano P., 2011, ApJ, 735, 104

Krimm H. A., et al., 2012, The Astronomer's Telegram, 4130, 1

Krimm H. A., et al., 2013a, ApJS, 209, 14

Krimm H. A., et al., 2013b, The Astronomer's Telegram, 4769, 1

Krivonos R., Vikhlinin A., Churazov E., Lutovinov A., Molkov S., Sunyaev R., 2005, ApJ, 625, 89

Krivonos R., Revnivtsev M., Churazov E., Sazonov S., Grebenev S., Sunyaev R., 2007a, A\&A, 463, 957

Krivonos R., Revnivtsev M., Lutovinov A., Sazonov S., Churazov E., Sunyaev R., 2007b, A\&A, 475, 775

Krivonos R., Revnivtsev M., Lutovinov A., Sazonov S., Churazov E., Sunyaev R., 2007c, A\&A, 475, 775

Krivonos R., Tsygankov S., Sunyaev R., Melnikov S., Bikmaev I., Pavlinsky M., Burenin R., 2009, The Astronomer's Telegram, 2170, 1

Krivonos R., Revnivtsev M., Tsygankov S., Sazonov S., Vikhlinin A., Pavlinsky M., Churazov E., Sunyaev R., 2010a, A\&A, 519, A107

Krivonos R., Tsygankov S., Revnivtsev M., Grebenev S., Churazov E., Sunyaev R., 2010b, A\&A, 523, A61

Krivonos R., Tsygankov S., Revnivtsev M., Grebenev S., Churazov E., Sunyaev R., 2010c, A\&A, 523, A61

Krivonos R., Tsygankov S., Burenin R., Revnivtsev M., Lutovinov A., 2011, The Astronomer's Telegram, 3382, 1

Krivonos R., Tsygankov S., Lutovinov A., Revnivtsev M., Churazov E., Sunyaev R., 2012, A\&A, 545, A27

Krivonos R., Lutovinov A., Molkov S., Revnivtsev M., Tsygankov S., Sunyaev R., 2013, The Astronomer's Telegram, 4924, 1

Krivonos R., Tsygankov S., Lutovinov A., Revnivtsev M., Churazov E., Sunyaev R., 2015, MNRAS, 448, 3766

Krivonos R. A., Tsygankov S. S., Mereminskiy I. A., Lutovinov A. A., Sazonov S. Y., Sunyaev R. A., 2017, MNRAS, 470, 512

Krivonos R., Sazonov S., Tsygankov S. S., Poutanen J., 2018, MNRAS, 480, 2357

Krivonos R. A., et al., 2021, New Astron. Rev., 92, 101612

Kuiper L., Hermsen W., 2009, A\&A, 501, 1031

Kuiper L., Hermsen W., in't Zand J., den Hartog P. R., 2005, The Astronomer's Telegram, 662, 1

Kuiper L., Keek S., Hermsen W., Jonker P. G., Steeghs D., 2006a, The Astronomer's Telegram, 684, 1

Kuiper L., den Hartog P. R., Hermsen W., 2006b, The Astronomer's Telegram, 939,1

Kuiper L., Jonker P. G., Torres M. A. P., Rest A., Keek S., 2008, The Astronomer's Telegram, 1774, 1

Kuulkers E., Lutovinov A., Parmar A., Capitanio F., Mowlavi N., Hermsen W., 2003, The Astronomer's Telegram, 149, 1

Kuulkers E., et al., 2006, The Astronomer's Telegram, 874, 1

Kuulkers E., et al., 2013, The Astronomer's Telegram, 4804, 1 
Kuznetsova E., Krivonos R., Churazov E., Lyskova N., Lutovinov A., 2019, MNRAS, 489, 1828

Kuznetsova E., Krivonos R., Lutovinov A., Clavel M., 2022, MNRAS, 509, 1605

La Parola V., Cusumano G., Segreto A., D’Aì A., Masetti N., D’Elia V., 2013, ApJ, 775, L24

Lamperti I., et al., 2017, MNRAS, 467, 540

Landi R., et al., 2007, The Astronomer's Telegram, 1288, 1

Landi R., et al., 2009, A\&A, 493, 893

Landi R., Bassani L., Malizia A., Stephen J. B., Bazzano A., Fiocchi M., Bird A. J., 2010a, MNRAS, 403, 945

Landi R., et al., 2010b, The Astronomer's Telegram, 2853, 1

Landi R., Malizia A., Bazzano A., Fiocchi M., Bird A. J., Gehrels N., 2011a, The Astronomer's Telegram, 3184, 1

Landi R., Bassani L., Masetti N., Bazzano A., Bird A. J., 2011b, The Astronomer's Telegram, 3271, 1

Landi R., Bassani L., Masetti N., Bazzano A., Bird A. J., 2011c, The Astronomer's Telegram, 3272, 1

Landi R., Bassani L., Masetti N., Bazzano A., Tarana A., Bird A. J., 2012, The Astronomer's Telegram, 4233, 1

Landi R., et al., 2017, MNRAS, 470, 1107

Lebrun F., et al., 2003, A\&A, 411, L141

Leyder J. C., Walter R., Rauw G., 2008, A\&A, 477, L29

Lubiński P., Cadolle Bel M., von Kienlin A., Budtz-Jorgensen C., McBreen B., Kretschmar P., Hermsen W., Shtykovsky P., 2005, The Astronomer's Telegram, 469, 1

Lutovinov A. A., Revnivtsev M. G., 2003, Astronomy Letters, 29, 719

Lutovinov A., Shaw S., Foschini L., Paul J., 2003a, The Astronomer's Telegram, 154,1

Lutovinov A., Walter R., Belanger G., Lund N., Grebenev S., Winkler C., 2003b, The Astronomer's Telegram, 155, 1

Lutovinov A., Cadolle Bel M., Belanger G., Goldwurm A., Budtz-Jorgensen C., Mowlavi N., Paul J., Orr A., 2004a, The Astronomer's Telegram, 328, 1

Lutovinov A., Rodrigues J., Budtz-Jorgensen C., Grebenev S., Winkler C., 2004b, The Astronomer's Telegram, 329, 1

Lutovinov A., Rodriguez J., Revnivtsev M., Shtykovskiy P., 2005a, A\&A, 433, L41

Lutovinov A., Revnivtsev M., Gilfanov M., Shtykovskiy P., Molkov S., Sunyaev R., 2005b, A\&A, 444, 821

Lutovinov A. A., Burenin R. A., Revnivtsev M. G., Suleimanov V. F., Tkachenko A. Y., 2010a, Astronomy Letters, 36, 904

Lutovinov A., Burenin R., Sazonov S., Revnivtsev M., Moiseev A., Dodonov S., 2010b, The Astronomer's Telegram, 2759, 1

Lutovinov A. A., Burenin R. A., Revnivtsev M. G., Bikmaev I. F., 2012, Astronomy Letters, 38, 1

Lutovinov A. A., Mironov A. I., Burenin R. A., Revnivtsev M. G., Tsygankov S. S., Pavlinsky M. N., Korobtsev I. V., Eselevich M. V., 2013, Astronomy Letters, 39, 513

Lutovinov A., Suleimanov V., Manuel Luna G. J., Sazonov S., de Martino D., Ducci L., Doroshenko V., Falanga M., 2020, New Astron. Rev., 91, 101547

Malizia A., et al., 2005, ApJ, 630, L157

Malizia A., et al., 2007, ApJ, 668, 81

Malizia A., Bassani L., Sguera V., Stephen J. B., Bazzano A., Fiocchi M., Bird A. J., 2010, MNRAS, 408, 975

Malizia A., Bassani L., Bazzano A., Bird A. J., Masetti N., Panessa F., Stephen J. B., Ubertini P., 2012, MNRAS, 426, 1750

Malizia A., Landi R., Molina M., Bassani L., Bazzano A., Bird A. J., Ubertini P., 2016, MNRAS, 460, 19

Malizia A., Bassani L., Sguera V., Bazzano A., Fiocchi M. T., Ubertini P., Bird A. J., 2017, The Astronomer's Telegram, 10411, 1

Malizia A., Sazonov S., Bassani L., Pian E., Beckmann V., Molina M., Mereminskiy I., Belanger G., 2020, New Astron. Rev., 90, 101545

Margutti R., et al., 2019, ApJ, 872, 18

Markwardt C. B., 2008, The Astronomer's Telegram, 1686, 1

Markwardt C. B., Swank J. H., Strohmayer T. E., 2004, The Astronomer's Telegram, 353, 1
Martí J., Paredes J. M., Bloom J. S., Casares J., Ribó M., Falco E. E., 2004, A\&A, 413, 309

Maselli A., et al., 2013, ApJS, 206, 17

Masetti N., Palazzi E., Bassani L., Malizia A., Stephen J. B., 2004, A\&A, 426, L41

Masetti N., et al., 2006a, A\&A, 449, 1139

Masetti N., et al., 2006b, A\&A, 455, 11

Masetti N., et al., 2006c, A\&A, 459, 21

Masetti N., Bassani L., Dean A. J., Ubertini P., Walter R., 2006d, The Astronomer's Telegram, 715, 1

Masetti N., Morelli L., Palazzi E., Stephen J., Bazzano A., Dean A. J., Walter R., Minniti D., 2006e, The Astronomer's Telegram, 783, 1

Masetti N., Rigon E., Maiorano E., Cusumano G., Palazzi E., Orlandini M., Amati L., Frontera F., 2007a, A\&A, 464, 277

Masetti N., et al., 2007b, A\&A, 470, 331

Masetti N., et al., 2007c, The Astronomer's Telegram, 1034, 1

Masetti N., et al., 2008, A\&A, 482, 113

Masetti N., et al., 2009, A\&A, 495, 121

Masetti N., et al., 2010a, A\&A, 511, A48

Masetti N., et al., 2010b, A\&A, 519, A96

Masetti N., et al., 2012a, A\&A, 538, A123

Masetti N., Nucita A. A., Parisi P., 2012b, A\&A, 544, A114

Masetti N., Jimenez-Bailon E., Chavushyan V., Parisi P., Bazzano A., Landi R., Bird A. J., 2012c, The Astronomer's Telegram, 4248, 1

Masetti N., et al., 2013, A\&A, 556, A120

McCollum B., Laine S., 2019, The Astronomer's Telegram, 13211, 1

Mereminskiy I. A., Krivonos R. A., Lutovinov A. A., Sazonov S. Y., Revnivtsev M. G., Sunyaev R. A., 2016, MNRAS, 459, 140

Mescheryakov A., Burenin R., Sazonov S., Revnivtsev M., Bikmaev I., Sunyaev R., 2006, The Astronomer's Telegram, 948, 1

Mescheryakov A., Burenin R., Sazonov S., Revnivtsev M., Bikmaev I., Pavlinsky M., Sunyaev R., 2009, The Astronomer's Telegram, 2132, 1

Milisavljevic D., Fesen R. A., Parrent J. T., Thorstensen J. R., 2011, The Astronomer's Telegram, 3146, 1

Miyasaka H., Tomsick J. A., Xu Y., Harrison F. A., 2018, The Astronomer's Telegram, 12340, 1

Molina M., Venturi T., Malizia A., Bassani L., Dallacasa D., Lal D. V., Bird A. J., Ubertini P., 2015, MNRAS, 451, 2370

Molkov S., Mowlavi N., Goldwurm A., Strong A., Lund N., Paul J., Oosterbroek T., 2003, The Astronomer's Telegram, 176, 1

Molkov S. V., Cherepashchuk A. M., Lutovinov A. A., Revnivtsev M. G., Postnov K. A., Sunyaev R. A., 2004, Astronomy Letters, 30, 534

Morelli L., Masetti N., Bassani L., Landi R., Malizia A., Bird A. J., Ubertini P., Galaz G., 2006, The Astronomer's Telegram, 785, 1

Morgan E., Swank J., Markwardt C., Gehrels N., 2005, The Astronomer's Telegram, 550, 1

Mori K., et al., 2015, ApJ, 814, 94

Nabizadeh A., Tsygankov S. S., Karasev D. I., Mönkkönen J., Lutovinov A. A., Nagirner D. I., Poutanen J., 2019, A\&A, 622, A198

Nakahira S., et al., 2013, The Astronomer's Telegram, 5474, 1

Natalucci L., Fiocchi M., Bazzano A., Kuulkers E., Sanchez C., 2011, The Astronomer's Telegram, 3181, 1

Nebot Gómez-Morán A., Motch C., Pineau F. X., Carrera F. J., Pakull M. W., Riddick F., 2015, MNRAS, 452, 884

Negoro H., et al., 2018, The Astronomer's Telegram, 12254, 1

Negueruela I., Schurch M. P. E., 2007, A\&A, 461, 631

Negueruela I., Smith D. M., Chaty S., 2005, The Astronomer's Telegram, 470,1

Negueruela I., Torrejón J. M., McBride V., 2007, The Astronomer's Telegram, 1239,1

Nespoli E., Fabregat J., Mennickent R. E., 2008a, A\&A, 486, 911

Nespoli E., Fabregat J., Mennickent R. E., 2008b, The Astronomer's Telegram, 1396, 1

Nespoli E., Fabregat J., Mennickent R. E., 2010, A\&A, 516, A94

Neustroev V. V., Veledina A., Poutanen J., Zharikov S. V., Tsygankov S. S., Sjoberg G., Kajava J. J. E., 2014, MNRAS, 445, 2424

Nucita A. A., Carpano S., Guainazzi M., 2007, A\&A, 474, L1 
Nucita A. A., De Paolis F., Saxton R., Read A. M., 2012, New Astron., 17, 589

Ochsenbein F., Bauer P., Marcout J., 2000, A\&AS, 143, 23

Oda S., et al., 2019, PASJ, 71, 108

Oh K., Yi S. K., Schawinski K., Koss M., Trakhtenbrot B., Soto K., 2015, ApJS, 219, 1

Oh K., et al., 2018, ApJS, 235, 4

Paizis A., et al., 2007, ApJ, 657, L109

Papitto A., Ferrigno C., Bozzo E., Gibaud L., Burderi L., di Salvo T., Riggio A., 2011, The Astronomer's Telegram, 3556, 1

Parikh A. S., Russell T. D., Wijnands R., Miller-Jones J. C. A., Sivakoff G. R., Tetarenko A. J., 2019, ApJ, 878, L28

Parisi P., et al., 2008, The Astronomer's Telegram, 1540, 1

Parisi P., et al., 2012, The Astronomer's Telegram, 4151, 1

Parisi P., et al., 2014, A\&A, 561, A67

Pavan L., Bozzo E., Ferrigno C., Ricci C., Manousakis A., Walter R., Stella L., 2011, A\&A, 526, A122

Pavlinsky M. N., Grebenev S. A., Sunyaev R. A., 1994, ApJ, 425, 110

Pavlinsky M., et al., 2021, arXiv e-prints, p. arXiv:2107.05879

Pearlman A. B., Coley J. B., Corbet R. H. D., Pottschmidt K., 2019, ApJ, 873, 86

Produit N., Ballet J., Mowlavi N., 2004, The Astronomer's Telegram, 278, 1

Rahoui F., Tomsick J. A., Krivonos R., 2017, MNRAS, 465, 1563

Ratti E. M., Bassa C. G., Torres M. A. P., Kuiper L., Miller-Jones J. C. A., Jonker P. G., 2010, MNRAS, 408, 1866

Rau A., 2018, The Astronomer's Telegram, 11332, 1

Renaud M., et al., 2010, ApJ, 716, 663

Revnivtsev M. G., Sazonov S. Y., Gilfanov M. R., Sunyaev R. A., 2003a, Astronomy Letters, 29, 587

Revnivtsev M., Chernyakova M., Capitanio F., Westergaard N. J., Shoenfelder V., Gehrels N., Winkler C., 2003b, The Astronomer's Telegram, 132, 1

Revnivtsev M., Tuerler M., Del Santo M., Westergaard N. J., Gehrels N., Winkler C., 2003c, IAU Circ., 8097, 2

Revnivtsev M. G., et al., 2004a, Astronomy Letters, 30, 382

Revnivtsev M. G., et al., 2004b, A\&A, 425, L49

Revnivtsev M. G., Sazonov S. Y., Molkov S. V., Lutovinov A. A., Churazov E. M., Sunyaev R. A., 2006, Astronomy Letters, 32, 145

Revnivtsev M., Sunyaev R., Lutovinov A., Sazonov S., 2007, The Astronomer's Telegram, 1253, 1

Revnivtsev M., Lutovinov A., Churazov E., Sazonov S., Gilfanov M., Grebenev S., Sunyaev R., 2008, A\&A, 491, 209

Revnivtsev M. G., et al., 2009, Astronomy Letters, 35, 33

Reynolds M. T., et al., 2012, The Astronomer's Telegram, 3951, 1

Ricci C., et al., 2017, ApJS, 233, 17

Rodes-Roca J. J., Bernabeu G., Magazzù A., Torrejón J. M., Solano E., 2018, MNRAS, 476, 2110

Rodriguez J., et al., 2004, The Astronomer's Telegram, 340, 1

Rodriguez J., Cadolle Bel M., Tomsick J. A., Corbel S., Brocksopp C., Paizis A., Shaw S. E., Bodaghee A., 2007, ApJ, 655, L97

Rodriguez J., Tomsick J. A., Chaty S., 2008, A\&A, 482, 731

Rodriguez J., Tomsick J. A., Chaty S., 2009, A\&A, 494, 417

Rodriguez J., Tomsick J. A., Bodaghee A., 2010, A\&A, 517, A14

Rojas A. F., et al., 2017, A\&A, 602, A124

Russell D. M., Lewis F., Altamirano D., Roche P., 2011, The Astronomer's Telegram, 3622, 1

Russell T. D., et al., 2019, ApJ, 883, 198

Sanchez-Fernandez C., Eckert D., Bozzo E., Kajava J., Kuulkers E., Chenevez J., 2015, The Astronomer's Telegram, 7946, 1

Sanna A., et al., 2017, A\&A, 598, A34

Sanna A., et al., 2018, A\&A, 617, L8

Saxton R. D., Read A. M., Esquej P., Freyberg M. J., Altieri B., Bermejo D., 2008, A\&A, 480, 611

Sazonov S., Churazov E., Revnivtsev M., Vikhlinin A., Sunyaev R., 2005, A\&A, 444, L37

Sazonov S., Revnivtsev M., Krivonos R., Churazov E., Sunyaev R., 2007, A\&A, 462, 57

Sazonov S., Revnivtsev M., Burenin R., Churazov E., Sunyaev R., Forman W. R., Murray S. S., 2008, A\&A, 487, 509
Sazonov S., et al., 2020, New Astron. Rev., 88, 101536

Scott D. M., Finger M. H., Wilson R. B., Koh D. T., Prince T. A., Vaughan B. A., Chakrabarty D., 1997, ApJ, 488, 831

Segreto A., Cusumano G., La Parola V., D’Aì A., Masetti N., D’Avanzo P., 2013, A\&A, 557, A113

Sguera V., Drave S. P., Sidoli L., Masetti N., Landi R., Bird A. J., Bazzano A., 2013, A\&A, 556, A27

Sguera V., Sidoli L., Bird A. J., Paizis A., Bazzano A., 2020, MNRAS, 491, 4543

Shaw A. W., Heinke C. O., Degenaar N., Wijnands R., Kaur R., Forestell L. M., 2017, MNRAS, 471, 2508

Shidatsu M., et al., 2018, ApJ, 868, 54

Sidoli L., Paizis A., Mereghetti S., 2006, A\&A, 450, L9

Sidoli L., Paizis A., Mereghetti S., Götz D., Del Santo M., 2011, MNRAS, 415,2373

Smith N., Hartigan P., 2006, ApJ, 638, 1045

Soldi S., Brandt S., Garau A. D., Grebenev S. A., Kuulkers E., Palumbo G. G. C., Tarana A., 2005, The Astronomer's Telegram, 456, 1

Soldi S., et al., 2006, The Astronomer's Telegram, 885, 1

Spiro S., et al., 2013, The Astronomer's Telegram, 5537, 1

Steeghs D., Knigge C., Drew J., Unruh Y., Greimel R., 2008, The Astronomer's Telegram, 1653, 1

Stephen J. B., Bassani L., Malizia A., Masetti N., Ubertini P., 2018, The Astronomer's Telegram, 11341, 1

Sunyaev R., Lutovinov A., Molkov S., Deluit S., 2003a, The Astronomer's Telegram, 181, 1

Sunyaev R. A., Grebenev S. A., Lutovinov A. A., Rodriguez J., Mereghetti S., Gotz D., Courvoisier T., 2003b, The Astronomer's Telegram, 190, 1

Terrier R., Mattana F., Djannati-Atai A., Marandon V., Renaud M., Dubois F., 2008, in Aharonian F. A., Hofmann W., Rieger F., eds, American Institute of Physics Conference Series Vol. 1085, American Institute of Physics Conference Series. pp 312-315, doi:10.1063/1.3076669

Terrier R., et al., 2010, ApJ, 719, 143

Tomsick J. A., Lingenfelter R., Walter R., Rodriguez J., Goldwurm A., Corbel S., Kaaret P., 2003, IAU Circ., 8076, 1

Tomsick J. A., Lingenfelter R., Corbel S., Goldwurm A., Kaaret P., 2004, The Astronomer's Telegram, 224, 1

Tomsick J. A., Chaty S., Rodriguez J., Foschini L., Walter R., Kaaret P., 2006, ApJ, 647, 1309

Tomsick J. A., Chaty S., Rodriguez J., Walter R., Kaaret P., 2008, ApJ, 685, 1143

Tomsick J. A., Chaty S., Rodriguez J., Walter R., Kaaret P., 2009, ApJ, 701, 811

Tomsick J. A., Bodaghee A., Chaty S., Rodriguez J., Rahoui F., Halpern J., Kalemci E., Özbey Arabaci M., 2012, ApJ, 754, 145

Tomsick J. A., Krivonos R., Rahoui F., Ajello M., Rodriguez J., Barrière N., Bodaghee A., Chaty S., 2015, MNRAS, 449, 597

Tomsick J. A., Rahoui F., Krivonos R., Clavel M., Strader J., Chomiuk L., 2016a, MNRAS, 460, 513

Tomsick J. A., Krivonos R., Wang Q., Bodaghee A., Chaty S., Rahoui F., Rodriguez J., Fornasini F. M., 2016b, ApJ, 816, 38

Tomsick J. A., et al., 2020, ApJ, 889, 53

Tomsick J. A., et al., 2021, ApJ, 914, 48

Torrejón J. M., Negueruela I., Smith D. M., Harrison T. E., 2010, A\&A, 510, A61

Torres M. A. P., Garcia M. R., McClintock J. E., Steeghs D., Miller J., Callanan P. J., Zhao P., Berlind P., 2004, The Astronomer's Telegram, 264,1

Torres M. A. P., Steeghs D., Garcia M. R., McClintock J. E., Berlind P., Zhao P., Jonker P. G., Callanan P. J., 2006, The Astronomer's Telegram, 862, 1

Tovmassian G., et al., 2017, A\&A, 608, A36

Townsend L. J., et al., 2011, MNRAS, 410, 1813

Tueller J., et al., 2005, The Astronomer's Telegram, 669, 1

Tueller J., Mushotzky R. F., Barthelmy S., Cannizzo J. K., Gehrels N., Markwardt C. B., Skinner G. K., Winter L. M., 2008, ApJ, 681, 113

Tueller J., et al., 2010, ApJS, 186, 378

Tuerler M., Walter R., Ferrigno C., 2012, The Astronomer's Telegram, 4183, 1 
Ubertini P., et al., 2003, A\&A, 411, L131

Ubertini P., et al., 2005, ApJ, 629, L109

Vasilopoulos G., Maggi P., Haberl F., Sturm R., Pietsch W., Bartlett E. S., Coe M. J., 2013, A\&A, 558, A74

Vasilopoulos G., Haberl F., Sturm R., Maggi P., Udalski A., 2014, A\&A, 567, A129

Voges W., et al., 1999, A\&A, 349, 389

Vovk I., et al., 2012, The Astronomer's Telegram, 4381, 1

Walter R., et al., 2004, The Astronomer's Telegram, 229, 1

Walter R., et al., 2006, A\&A, 453, 133

Wang W., 2010, A\&A, 516, A15

Watson M. G., et al., 2009, A\&A, 493, 339

Webb N. A., et al., 2020, A\&A, 641, A136

Westergaard N. J., Budtz-Jorgensen C., Chenevez J., Lund N., Brandt S., Oxborrow C. A., 2006, The Astronomer's Telegram, 967, 1

Wijnands R., 2006, The Astronomer's Telegram, 972, 1

Winkler C., et al., 2003, A\&A, 411, L1

Winter L. M., Mushotzky R. F., Tueller J., Markwardt C., 2008, ApJ, 674, 686

Winter L. M., Mushotzky R. F., Terashima Y., Ueda Y., 2009, ApJ, 701, 1644

Worpel H., Schwope A. D., Traulsen I., Mukai K., Ok S., 2020, A\&A, 639, A17

Xiao G. C., et al., 2019, Journal of High Energy Astrophysics, 24, 30

Xu X., Shao Y., Li X.-D., 2019, MNRAS, 489, 3031

Yatabe F., et al., 2019, The Astronomer's Telegram, 12425, 1

Zhang S., Chen Y. P., Wang J. M., Torres D. F., Li T. P., 2009, A\&A, 502, 231

Zhang S., et al., 2015, ApJ, 815, 132

Zolotukhin I. Y., Revnivtsev M. G., 2011, MNRAS, 411, 620

Zolotukhin I. Y., Revnivtsev M. G., 2015, MNRAS, 446, 2418

Zurita Heras J. A., Chaty S., 2008, A\&A, 489, 657

Zurita Heras J. A., Chaty S., Tomsick J. A., 2009, A\&A, 502, 787

de Rosa A., et al., 2012, MNRAS, 420, 2087

den Hartog P. R., Hermsen W., Kuiper L. M., in't Zand J. J. M., Winkler C., Domingo A., 2004a, The Astronomer's Telegram, 261, 1

den Hartog P. R., Kuiper L. M., Corbet R. H. D., in't Zand J. J. M., Hermsen W., Vink J., Remillard R., van der Klis M., 2004b, The Astronomer's Telegram, 281, 1

den Hartog P. R., Hermsen W., Kuiper L., Vink J., in't Zand J. J. M., Collmar W., 2006, A\&A, 451, 587

in 't Zand J., Bazzano A., Cocchi M., Ubertini P., Muller J. M., Torroni V., 1998, IAU Circ., 6846, 2

in't Zand J. J. M., 2005, A\&A, 441, L1

\section{APPENDIX A: CATALOG OF SOURCES IN 17-60 KEV}

BAND 
Table A1. The full list of hard X-ray sources detected during the INTEGRAL all-sky survey based on 17 years of observations. This catalog is only available in the online version of the paper, at the CDS via anonymous ftp to cdsarc.u-strasbg.fr (130.79.128.5) or via http://cdsarc.u-strasbg.fr/viz-bin/qcat?, and at website http://integral. cosmos.ru.

\begin{tabular}{|c|c|c|c|c|c|c|}
\hline Id & Name $^{1}$ & $\begin{array}{l}\mathrm{RA}^{2} \\
(\mathrm{deg})\end{array}$ & $\begin{array}{l}\operatorname{Dec}^{2} \\
(\mathrm{deg})\end{array}$ & $\begin{array}{c}\text { Flux }^{3} \\
(17-60 \mathrm{keV})\end{array}$ & Type $^{4}$ & Notes $^{5}$ \\
\hline N001 & SWIFT J0001.6-7701 & 0.370 & -77.020 & $0.58 \pm 0.12$ & SEYFERT & $\mathrm{z}=0.058 ;$ !B16; \\
\hline N002 & IGR J00040+7020 & 1.029 & 70.315 & $0.86 \pm 0.08$ & SEYFERT & R $1 ; z=0.096$ \\
\hline N003 & IGR J00115+2645 & 2.880 & 26.760 & $1.90 \pm 0.40$ & UNIDENT & !B 16 \\
\hline N004 & IGR J00197+6224 & 4.930 & 62.410 & $0.29 \pm 0.06$ & UNIDENT & !B16; \\
\hline N005 & IGR J00234+6141 & 5.742 & 61.686 & $0.78 \pm 0.06$ & $\mathrm{CV}$ & R 2,3; \\
\hline N006 & TYCHO SNR & 6.325 & 64.150 & $0.76 \pm 0.06$ & SNR & \\
\hline N007 & IGR J00255+6821 & 6.407 & 68.362 & $0.53 \pm 0.07$ & SEYFERT & R 4,1; $\mathrm{z}=0.012$ \\
\hline N008 & V709 Cas & 7.201 & 59.296 & $5.52 \pm 0.06$ & $\mathrm{CV}$ & \\
\hline N009 & IGR J00291+5934 & 7.263 & 59.572 & $1.84 \pm 0.06$ & LMXB & R 5,6; \\
\hline N010 & IGR J00335+6126 & 8.372 & 61.469 & $0.65 \pm 0.06$ & SEYFERT & $\mathrm{R} 4,7 ; \mathrm{z}=0.105$ \\
\hline N011 & SWIFT J0034.5-7904 & 8.570 & -79.089 & $0.98 \pm 0.12$ & SEYFERT & $\mathrm{z}=0.074$ \\
\hline N012 & 1ES $0033+595$ & 8.970 & 59.822 & $1.76 \pm 0.06$ & BLAZAR & $\mathrm{z}=0.086$ \\
\hline N013 & IGR J00370+6122 & 9.275 & 61.397 & $1.24 \pm 0.06$ & HMXB & R 8; \\
\hline N014 & NGC 235A & 10.757 & -23.473 & $3.86 \pm 0.66$ & SEYFERT & $\mathrm{z}=0.022 ;$ !B16; \\
\hline N015 & MRK 348 & 12.181 & 31.951 & $6.07 \pm 0.23$ & SEYFERT & $\mathrm{z}=0.015$ \\
\hline N016 & RX J0053.8-7226 & 12.737 & -72.226 & $0.69 \pm 0.11$ & HMXB & in SMC; !B16; \\
\hline N017 & RX J0053.8-7226 & 13.507 & -72.470 & $0.70 \pm 0.10$ & HMXB & \\
\hline N018 & IGR J00555+4610 & 13.867 & 46.195 & $1.28 \pm 0.27$ & $\mathrm{CV}$ & \\
\hline N019 & Gamma Cas & 14.178 & 60.716 & $5.27 \pm 0.07$ & STAR & Be star; \\
\hline N020 & IGR J00569-7225 & 14.220 & -72.430 & $1.11 \pm 0.10$ & HMXB & R 9; in SMC; \\
\hline N021 & IGR J00569+6359 & 14.240 & 63.990 & $0.31 \pm 0.07$ & SEYFERT & R $10 ; z=0.291 ;$ !B16; \\
\hline N022 & MRK 0352 & 14.949 & 31.935 & $1.82 \pm 0.24$ & SEYFERT & $\mathrm{z}=0.015$ \\
\hline N023 & IGR J01017+6519 & 15.440 & 65.330 & $0.46 \pm 0.07$ & SEYFERT & R $11 ; z=0.085 ;$ !B16; \\
\hline N024 & IGR J01036-6439 & 15.920 & -64.660 & $0.91 \pm 0.14$ & BLAZAR & $\mathrm{z}=0.163 ; \mathrm{B} 16$ \\
\hline N025 & IGR J01044-7253 & 16.110 & -72.900 & $1.16 \pm 0.10$ & HMXB & $\mathrm{R} 12$; in $\mathrm{SMC}$; \\
\hline N026 & IGR J01062-2436 & 16.570 & -24.600 & $2.84 \pm 0.61$ & UNIDENT & !B16; \\
\hline N027 & SMC X-1 & 19.291 & -73.447 & $26.92 \pm 0.11$ & HMXB & in SMC; \\
\hline N028 & $1 \mathrm{~A} 0114+650$ & 19.512 & 65.288 & $11.21 \pm 0.08$ & HMXB & \\
\hline N029 & 4U $0115+63$ & 19.633 & 63.740 & $27.19 \pm 0.08$ & HMXB & \\
\hline N030 & FAIRALL 9 & 20.950 & -58.830 & $2.70 \pm 0.24$ & SEYFERT & $\mathrm{z}=0.048$ \\
\hline N031 & NGC 0526A & 20.998 & -35.052 & $3.22 \pm 0.56$ & SEYFERT & $\mathrm{z}=0.019$ \\
\hline N032 & IGR J01242+3348 & 21.060 & 33.800 & $1.36 \pm 0.28$ & SEYFERT & $\mathrm{z}=0.020 ;$ !B16; \\
\hline N033 & ESO 297- G 018 & 24.653 & -40.010 & $3.02 \pm 0.43$ & SEYFERT & $\mathrm{z}=0.025$ \\
\hline N034 & $4 \mathrm{U} 0142+61$ & 26.587 & 61.747 & $2.93 \pm 0.09$ & MAGNETAR & \\
\hline N035 & RX J0146.9+6121 & 26.712 & 61.359 & $2.02 \pm 0.09$ & HMXB & \\
\hline N036 & IGR J01528-0326 & 28.221 & -3.443 & $1.53 \pm 0.14$ & SEYFERT & R $13 ; z=0.017$ \\
\hline N037 & IGR J01545+6437 & 28.625 & 64.617 & $0.53 \pm 0.10$ & SEYFERT & R $14,15,16 ; z=0.035$ \\
\hline N038 & IGR J015712-7259 & 29.318 & -72.976 & $0.49 \pm 0.11$ & HMXB? & $\mathrm{R} 17 ;$ in $\mathrm{SMC}$ \\
\hline N039 & IGR J01583+6713 & 29.576 & 67.224 & $0.44 \pm 0.10$ & HMXB & R 18,19 \\
\hline N040 & NGC 788 & 30.280 & -6.816 & $4.66 \pm 0.13$ & SEYFERT & $\mathrm{z}=0.014$ \\
\hline N041 & MRK 1018 & 31.579 & -0.300 & $1.09 \pm 0.14$ & SEYFERT & $\mathrm{z}=0.043$ \\
\hline N042 & SWIFT J0208.4-7428 & 31.675 & -74.470 & $0.68 \pm 0.11$ & HMXB & in LMC; !B16; \\
\hline N043 & IGR J02086-1742 & 32.166 & -17.649 & $1.05 \pm 0.22$ & SEYFERT & R $14,20,15 ; z=0.129$ \\
\hline N044 & IGR J02095+5226 & 32.422 & 52.446 & $2.88 \pm 0.18$ & SEYFERT & $\mathrm{R} 21 ; \mathrm{z}=0.049$ \\
\hline N045 & IGR J02145+5142 & 33.559 & 51.691 & $1.06 \pm 0.19$ & BLAZAR & R $22 ; \mathrm{z}=0.049 ;$ !B 16 \\
\hline N046 & MRK 590 & 33.635 & -0.793 & $1.09 \pm 0.13$ & SEYFERT & $\mathrm{z}=0.026$ \\
\hline N047 & IGR J02164+5126 & 34.121 & 51.439 & $1.10 \pm 0.20$ & SEYFERT & R 7,23; z=0.422; \\
\hline N048 & QSO B0212+73 & 34.430 & 73.844 & $1.56 \pm 0.19$ & BLAZAR & $\mathrm{z}=2.367$ \\
\hline N049 & MRK 1040 & 37.053 & 31.315 & $3.60 \pm 0.29$ & SEYFERT & $\mathrm{z}=0.016$ \\
\hline N050 & IGR J02343+3229 & 38.564 & 32.491 & $4.33 \pm 0.23$ & SEYFERT & R 24,25; z=0.016; \\
\hline N051 & NGC 0985 & 38.648 & -8.829 & $1.76 \pm 0.14$ & SEYFERT & $\mathrm{z}=0.043$ \\
\hline N052 & SWIFT J0238.2-5213 & 39.550 & -52.270 & $1.12 \pm 0.25$ & SEYFERT & $\mathrm{z}=0.045 ; \mathrm{B} 16$ \\
\hline N053 & LSI +61303 & 40.100 & 61.218 & $1.51 \pm 0.13$ & HMXB & \\
\hline N054 & NGC 1052 & 40.268 & -8.246 & $1.45 \pm 0.15$ & SEYFERT & $\mathrm{z}=0.005$ \\
\hline N055 & NGC 1068 & 40.681 & -0.003 & $2.15 \pm 0.16$ & SEYFERT & $\mathrm{z}=0.004$ \\
\hline
\end{tabular}


Table A1 - continued from previous page

\begin{tabular}{|c|c|c|c|c|c|c|}
\hline Id & Name $^{1}$ & $\begin{array}{l}\mathrm{RA}^{2} \\
(\mathrm{deg})\end{array}$ & $\begin{array}{l}\operatorname{Dec}^{2} \\
(\mathrm{deg})\end{array}$ & $\begin{array}{c}\text { Flux }^{3} \\
(17-60 \mathrm{keV})\end{array}$ & Type $^{4}$ & Notes \\
\hline N056 & SWIFT J0243.6+6124 & 40.918 & 61.434 & $4.56 \pm 0.13$ & HMXB & R 26,27,28; !B16; \\
\hline N057 & $4 \mathrm{U} 0241+61$ & 41.239 & 62.458 & $4.99 \pm 0.14$ & SEYFERT & R $29 ; z=0.045$ \\
\hline N058 & SWIFT J0250.2+4650 & 42.606 & 46.802 & $1.36 \pm 0.16$ & SEYFERT & R 30,$31 ; z=0.021 ;$ !B 16 \\
\hline N059 & IGR J02501+5440 & 42.677 & 54.705 & $1.25 \pm 0.15$ & SEYFERT & $\mathrm{R} 4,1 ; \mathrm{z}=0.015$ \\
\hline N060 & IGR J02524-0829 & 43.099 & -8.489 & $1.52 \pm 0.18$ & SEYFERT & R 32; z=0.017; \\
\hline N061 & NGC 1142 & 43.794 & -0.193 & $4.45 \pm 0.20$ & SEYFERT & $\mathrm{z}=0.029$ \\
\hline N062 & XY Ari & 44.037 & 19.468 & $2.87 \pm 0.32$ & $\mathrm{CV}$ & \\
\hline N063 & MCG-02-08-038 & 45.000 & -10.800 & $1.04 \pm 0.23$ & SEYFERT & $\mathrm{z}=0.032$ \\
\hline N064 & NGC 1194 & 46.003 & -1.106 & $1.69 \pm 0.25$ & SEYFERT & $\mathrm{z}=0.013$ \\
\hline N065 & SWIFT J0308.5-7251 & 46.897 & -72.834 & $0.72 \pm 0.11$ & SEYFERT & $\mathrm{z}=0.028 ; \mathrm{B} 16$ \\
\hline N066 & IGR J03088+3659 & 47.220 & 36.990 & $0.59 \pm 0.13$ & UNIDENT & !B 16 \\
\hline N067 & IGR J03117+5028 & 47.945 & 50.466 & $0.91 \pm 0.14$ & SEYFERT & R 22,$33 ; z=0.062 ;$ !B 16 \\
\hline N068 & SWIFT J0318.7+6828 & 49.658 & 68.465 & $0.92 \pm 0.21$ & SEYFERT & $\mathrm{z}=0.090$ \\
\hline N069 & Perseus & 49.964 & 41.519 & $4.81 \pm 0.13$ & CLUSTER & $\mathrm{z}=0.018 ; \mathrm{C} ; \mathrm{E}$ \\
\hline N070 & 1 H $0323+342$ & 51.172 & 34.179 & $1.52 \pm 0.13$ & SEYFERT & $\mathrm{z}=0.063$ \\
\hline N071 & IGR J03249+4041 & 51.224 & 40.698 & $0.76 \pm 0.12$ & SEYFERT & R 34,$35 ; z=0.048 ;$ !B16; \\
\hline N072 & GK Per & 52.796 & 43.886 & $4.11 \pm 0.12$ & $\mathrm{CV}$ & \\
\hline N073 & IGR J03334+3718 & 53.348 & 37.300 & $1.20 \pm 0.12$ & SEYFERT & R 36,24; z=0.055; \\
\hline N074 & NGC 1365 & 53.424 & -36.170 & $4.21 \pm 0.52$ & SEYFERT & $\mathrm{z}=0.006$ \\
\hline N075 & V0332+53 & 53.750 & 53.173 & $162.68 \pm 0.13$ & HMXB & \\
\hline N076 & 4C 32.14 & 54.115 & 32.312 & $2.08 \pm 0.13$ & BLAZAR & $\mathrm{z}=1.258$ \\
\hline N077 & ESO $548-81$ & 55.474 & -21.257 & $3.12 \pm 0.50$ & SEYFERT & $\mathrm{z}=0.015$ \\
\hline N078 & IGR J03526-6830 & 58.150 & -68.510 & $0.45 \pm 0.11$ & BLAZAR & $\mathrm{z}=0.087$ \\
\hline N079 & SWIFT J0353.7+3711 & 58.400 & 37.200 & $0.60 \pm 0.12$ & SEYFERT & $\mathrm{z}=0.018$ \\
\hline N080 & $4 \mathrm{U} 0352+30$ & 58.846 & 31.041 & $40.23 \pm 0.14$ & HMXB & \\
\hline N081 & IGR J03574-6602 & 59.375 & -66.043 & $0.61 \pm 0.12$ & UNIDENT & R 37,38; !B16; \\
\hline N082 & SWIFT J0359.7+5058 & 59.931 & 50.965 & $0.89 \pm 0.12$ & BLAZAR & $\mathrm{z}=1.520 ; \mathrm{B} 16$ \\
\hline N083 & IGR J04059+5416 & 61.500 & 54.280 & $0.88 \pm 0.13$ & AGN & R 39; !B16; \\
\hline N084 & IGR J04072+0342 & 61.822 & 3.700 & $1.21 \pm 0.22$ & SEYFERT & $\mathrm{z}=0.089$ \\
\hline N085 & IGR J04085-6546 & 62.150 & -65.770 & $0.54 \pm 0.11$ & AGN & $\mathrm{R} 40 ; \mathrm{z}=0.125 ;$ !B 16 \\
\hline N086 & SWIFT J0414.8-0754 & 63.750 & -7.920 & $1.45 \pm 0.28$ & SEYFERT & $\mathrm{z}=0.038 ; \mathrm{B} 16$ \\
\hline N087 & $3 \mathrm{C} 111$ & 64.582 & 38.021 & $6.03 \pm 0.13$ & SEYFERT & $\mathrm{z}=0.049$ \\
\hline N088 & NGC 1566 & 64.960 & -54.940 & $1.78 \pm 0.24$ & SEYFERT & $\mathrm{z}=0.005 ; \mathrm{B} 16$ \\
\hline N089 & IGR J04221+4856 & 65.530 & 48.950 & $0.67 \pm 0.12$ & SEYFERT & $\mathrm{R} 41,42 ; \mathrm{z}=0.114$ \\
\hline N090 & SWIFT J0422.7-5611 & 65.638 & -56.200 & $1.38 \pm 0.21$ & SEYFERT & $\mathrm{z}=0.043 ;$ !B 16 \\
\hline N091 & IGR J04236+0408 & 65.940 & 4.136 & $1.60 \pm 0.15$ & SEYFERT & $\mathrm{z}=0.046$ \\
\hline N092 & $1 \mathrm{H} 0419-577$ & 66.507 & -57.201 & $1.53 \pm 0.19$ & SEYFERT & $\mathrm{z}=0.104 ;$ !B 16 \\
\hline N093 & SWIFT J0427.0+0734 & 66.800 & 7.250 & $0.67 \pm 0.15$ & SEYFERT & $\mathrm{z}=0.097 ; \mathrm{!B} 16$ \\
\hline N094 & IGR J04288-6702 & 67.201 & -67.040 & $0.55 \pm 0.09$ & SEYFERT & $\mathrm{R} 43 ; \mathrm{z}=0.065 ; \mathrm{B} 16 ; \mathrm{C}$ \\
\hline N095 & ABELL 3266 & 67.854 & -61.441 & $0.73 \pm 0.12$ & CLUSTER & $\mathrm{z}=0.059 ; \mathrm{B} 16$ \\
\hline N096 & $3 \mathrm{C} 120$ & 68.304 & 5.354 & $5.64 \pm 0.12$ & SEYFERT & $\mathrm{z}=0.033$ \\
\hline N097 & IGR J04379-7240 & 69.492 & -72.669 & $0.61 \pm 0.09$ & UNIDENT & R 37; !B16; \\
\hline N098 & SWIFT J0440.2-5941 & 69.996 & -59.682 & $0.77 \pm 0.14$ & SEYFERT & $\mathrm{z}=0.058 ; \mathrm{B} 16$ \\
\hline N099 & RX J0440.9+4431 & 70.234 & 44.558 & $3.48 \pm 0.13$ & HMXB & \\
\hline N100 & PKS 0440-00 & 70.654 & -0.307 & $0.67 \pm 0.10$ & BLAZAR & $\mathrm{z}=0.845 ;$ !B16; \\
\hline N101 & UGC 3142 & 70.980 & 28.987 & $3.40 \pm 0.16$ & SEYFERT & $\mathrm{z}=0.022$ \\
\hline N102 & SWIFT J0451.5-6949 & 72.814 & -69.799 & $1.46 \pm 0.08$ & HMXB & in LMC; \\
\hline N103 & MCG -01-13-025 & 72.921 & -3.808 & $1.08 \pm 0.10$ & SEYFERT & $\mathrm{z}=0.016$ \\
\hline N104 & RX J0452.0+4932 & 73.034 & 49.549 & $2.35 \pm 0.13$ & SEYFERT & $\mathrm{z}=0.029$ \\
\hline N105 & CGCG 420-015 & 73.382 & 4.036 & $1.79 \pm 0.09$ & SEYFERT & $\mathrm{z}=0.029$ \\
\hline N106 & ESO 033-G002 & 73.963 & -75.542 & $1.23 \pm 0.09$ & SEYFERT & $\mathrm{z}=0.018$ \\
\hline N107 & IGR J04571+4527 & 74.300 & 45.458 & $0.93 \pm 0.13$ & $\mathrm{CV}$ & R 15,44; \\
\hline N108 & IGR J05007-7047 & 75.197 & -70.763 & $1.25 \pm 0.08$ & HMXB & R 45,46; in LMC; \\
\hline N109 & LEDA 075258 & 75.559 & 3.525 & $1.66 \pm 0.08$ & SEYFERT & $\mathrm{z}=0.016$ \\
\hline N110 & V1062 Tau & 75.634 & 24.746 & $1.31 \pm 0.11$ & $\mathrm{CV}$ & \\
\hline N111 & SWIFT J0504.6-7345 & 76.142 & -73.824 & $0.69 \pm 0.09$ & SEYFERT & $\mathrm{z}=0.045 ; \mathrm{B} 16$ \\
\hline $\mathrm{N} 112$ & IGR J05048-7340 & 76.194 & -73.667 & $0.38 \pm 0.09$ & SEYFERT & $\mathrm{z}=0.045 ; \mathrm{B} 16$ \\
\hline N113 & SWIFT J0505.6-6736 & 76.352 & -67.577 & $0.82 \pm 0.08$ & AGN & $\mathrm{z}=0.471 ; \mathrm{B} 16$ \\
\hline
\end{tabular}


Table A1 - continued from previous page

\begin{tabular}{|c|c|c|c|c|c|c|}
\hline Id & Name $^{1}$ & $\begin{array}{l}\mathrm{RA}^{2} \\
(\mathrm{deg})\end{array}$ & $\begin{array}{l}\operatorname{Dec}^{2} \\
(\mathrm{deg})\end{array}$ & $\begin{array}{c}\text { Flux }^{3} \\
(17-60 \mathrm{keV})\end{array}$ & Type $^{4}$ & Notes \\
\hline N114 & XSS J05054-2348 & 76.429 & -23.850 & $3.88 \pm 0.29$ & SEYFERT & $\mathrm{z}=0.035$ \\
\hline N115 & IGR J05081+1722 & 77.040 & 17.370 & $1.39 \pm 0.11$ & SEYFERT & $\mathrm{z}=0.018$ \\
\hline N116 & IGR J05099-6913 & 77.486 & -69.221 & $0.36 \pm 0.08$ & UNIDENT & R 43; !B16; \\
\hline N117 & IRAS $05078+1626$ & 77.684 & 16.497 & $4.89 \pm 0.11$ & SEYFERT & $\mathrm{z}=0.018$ \\
\hline N118 & $4 \mathrm{U} 0513-40$ & 78.523 & -40.064 & $3.42 \pm 0.33$ & LMXB & \\
\hline N119 & SWIFT J0515.3+1854 & 78.840 & 18.900 & $1.40 \pm 0.10$ & SEYFERT & $\mathrm{R} 47 ; \mathrm{z}=0.023$ \\
\hline N120 & ARK 120 & 79.040 & -0.145 & $4.09 \pm 0.08$ & SEYFERT & $\mathrm{z}=0.032$ \\
\hline N121 & IGR J05162-1034 & 79.070 & -10.570 & $0.88 \pm 0.14$ & SEYFERT & $\mathrm{z}=0.029 ; \mathrm{B} 16$ \\
\hline N122 & ESO $362-18$ & 79.900 & -32.657 & $2.84 \pm 0.23$ & SEYFERT & $\mathrm{z}=0.013$ \\
\hline N123 & PICTOR A & 79.937 & -45.770 & $2.51 \pm 0.47$ & SEYFERT & $\mathrm{z}=0.035$ \\
\hline N124 & LMC X-2 & 80.040 & -71.951 & $0.97 \pm 0.08$ & LMXB & in LMC; \\
\hline N125 & RX J0520.5-6932 & 80.123 & -69.532 & $0.57 \pm 0.08$ & HMXB & R 48; in LMC; !B16; \\
\hline N126 & PKS 0521-36 & 80.683 & -36.463 & $1.65 \pm 0.25$ & BLAZAR & $\mathrm{z}=0.055$ \\
\hline N127 & RX J0525.3+2413 & 81.392 & 24.218 & $0.80 \pm 0.09$ & $\mathrm{CV}$ & \\
\hline N128 & TV COL & 82.355 & -32.813 & $4.65 \pm 0.22$ & $\mathrm{CV}$ & \\
\hline N129 & IGR J05305-6559 & 82.548 & -65.857 & $0.49 \pm 0.08$ & HMXB & $\mathrm{R} 43 ;$ in $\mathrm{LMC}$ \\
\hline N130 & SWIFT J053041.9-665426 & 82.660 & -66.890 & $0.45 \pm 0.08$ & HMXB & R 49; in LMC; !B16; \\
\hline N131 & PKS $0528+134$ & 82.739 & 13.563 & $0.59 \pm 0.10$ & BLAZAR & $\mathrm{z}=2.060$ \\
\hline N132 & IGR J05305-6559 & 82.805 & -66.118 & $2.17 \pm 0.08$ & HMXB & R 43; in LMC; C; \\
\hline N133 & LMC X-4 & 83.192 & -66.368 & $30.56 \pm 0.08$ & HMXB & in $\mathrm{LMC}$ \\
\hline N134 & IGR J05329-7051 & 83.242 & -70.854 & $0.38 \pm 0.08$ & BLAZAR? & R $37 ; z=1.238 ;$ !B16; \\
\hline N135 & $\mathrm{Crab}$ & 83.632 & 22.018 & $1345.73 \pm 0.09$ & SNR / PULSAR & \\
\hline N136 & IGR J05342-6016 & 83.657 & -60.269 & $1.09 \pm 0.11$ & SEYFERT & $\mathrm{z}=0.057 ; \mathrm{B} 16$ \\
\hline N137 & TW Pic & 83.682 & -57.989 & $1.44 \pm 0.14$ & $\mathrm{CV}$ & \\
\hline N138 & IGR J05373-8424 & 84.342 & -84.408 & $0.99 \pm 0.19$ & UNIDENT & R 37; !B16; \\
\hline N139 & PSR J0537-6910 & 84.444 & -69.171 & $0.65 \pm 0.08$ & SNR / PULSAR & in LMC; !B16; C; \\
\hline N140 & LMC X-3 & 84.704 & -64.117 & $0.79 \pm 0.09$ & НMXB & in LMC; !B16; \\
\hline N141 & A $0535+262$ & 84.733 & 26.343 & $163.32 \pm 0.09$ & HMXB & $\mathrm{T}$ \\
\hline N142 & LMC X-1 & 84.911 & -69.746 & $2.24 \pm 0.08$ & HMXB & in LMC; \\
\hline N143 & PKS 0537-286 & 84.968 & -28.676 & $1.31 \pm 0.20$ & BLAZAR & $\mathrm{z}=3.104$ \\
\hline N144 & PSR B0540-69 & 85.001 & -69.338 & $2.69 \pm 0.08$ & SNR / PULSAR & in LMC; \\
\hline N145 & IGR J05414-6858 & 85.361 & -69.024 & $0.83 \pm 0.08$ & HMXB & in $\mathrm{LMC} ; \mathrm{C}$ \\
\hline N146 & XMMU J054134.7-682550 & 85.437 & -68.430 & $0.53 \pm 0.08$ & HMXB & in LMC; !B16; \\
\hline N147 & BY Cam & 85.729 & 60.847 & $2.28 \pm 0.30$ & $\mathrm{CV}$ & \\
\hline N148 & IGR J05470+5034 & 86.750 & 50.580 & $1.49 \pm 0.21$ & SEYFERT & R 31; z=0.036; \\
\hline N149 & NGC 2110 & 88.047 & -7.462 & $13.41 \pm 0.13$ & SEYFERT & $\mathrm{z}=0.008$ \\
\hline N150 & MCG+08-11-011 & 88.734 & 46.437 & $10.32 \pm 0.22$ & SEYFERT & $\mathrm{z}=0.021$ \\
\hline N151 & V405 Aur & 89.507 & 53.892 & $2.84 \pm 0.26$ & $\mathrm{CV}$ & \\
\hline N152 & SWIFT J0600.7+0008 & 90.190 & 0.100 & $0.67 \pm 0.12$ & SEYFERT & $\mathrm{z}=0.114 ;$ !B16; \\
\hline N153 & IRAS $05589+2828$ & 90.556 & 28.477 & $3.77 \pm 0.11$ & SEYFERT & $\mathrm{z}=0.033$ \\
\hline N154 & ESO 005- G 004 & 91.415 & -86.632 & $1.70 \pm 0.24$ & SEYFERT & $\mathrm{z}=0.006$ \\
\hline N155 & IGR J06058-2755 & 91.471 & -27.934 & $1.19 \pm 0.24$ & SEYFERT & R $15 ; z=0.089$ \\
\hline N156 & IGR J06075-6148 & 91.874 & -61.808 & $0.59 \pm 0.11$ & AGN & $\mathrm{z}=0.004 ;$ !B 16 \\
\hline N157 & MRK 3 & 93.901 & 71.040 & $7.90 \pm 0.20$ & SEYFERT & $\mathrm{z}=0.014$ \\
\hline N158 & 4U 0614+091 & 94.283 & 9.135 & $23.69 \pm 0.17$ & LMXB & \\
\hline N159 & IGR J06233-6436 & 95.776 & -64.598 & $0.61 \pm 0.09$ & BLAZAR & $\mathrm{z}=0.129 ;$ !B16; \\
\hline N160 & ESO 426-G 002 & 95.960 & -32.206 & $1.45 \pm 0.32$ & SEYFERT & $\mathrm{z}=0.022$ \\
\hline N161 & IGR J06239-6052 & 95.960 & -60.963 & $1.84 \pm 0.12$ & SEYFERT & R 50; z=0.040; \\
\hline N162 & IGR J06253+7334 & 96.303 & 73.551 & $1.43 \pm 0.18$ & $\mathrm{CV}$ & \\
\hline N163 & SWIFT J0634.7-7445 & 98.637 & -74.758 & $0.63 \pm 0.10$ & SEYFERT & $\mathrm{z}=0.112 ; \mathrm{B} 16$ \\
\hline N164 & IGR J06354-7516 & 98.858 & -75.282 & $0.77 \pm 0.10$ & BLAZAR & $\mathrm{z}=0.651$ \\
\hline N165 & IGR J06380-7536 & 99.430 & -75.646 & $0.63 \pm 0.10$ & SEYFERT & $\mathrm{z}=0.089 ; \mathrm{B} 16$ \\
\hline N166 & IGR J06402-2552 & 100.052 & -25.883 & $2.17 \pm 0.31$ & SEYFERT & $\mathrm{z}=0.025$ \\
\hline N167 & IGR J06415+3251 & 100.366 & 32.878 & $2.31 \pm 0.28$ & SEYFERT & $\mathrm{R} 51 ; \mathrm{z}=0.047$ \\
\hline N168 & IGR J06503-7742 & 102.477 & -77.704 & $0.76 \pm 0.12$ & AGN & $\mathrm{z}=0.037 ; \mathrm{B} 16$ \\
\hline N169 & MRK 6 & 103.054 & 74.425 & $3.22 \pm 0.15$ & SEYFERT & $\mathrm{z}=0.019$ \\
\hline N170 & FAIRALL 265 & 104.124 & -65.560 & $0.77 \pm 0.11$ & SEYFERT & $\mathrm{z}=0.030 ;$ !B 16 \\
\hline N171 & IGR J06571+7802 & 104.277 & 78.044 & $0.75 \pm 0.15$ & UNIDENT & R $37 ;$ !B16; \\
\hline
\end{tabular}


Table A1 - continued from previous page

\begin{tabular}{|c|c|c|c|c|c|c|}
\hline Id & Name $^{1}$ & $\begin{array}{l}\mathrm{RA}^{2} \\
(\mathrm{deg})\end{array}$ & $\begin{array}{l}\operatorname{Dec}^{2} \\
(\mathrm{deg})\end{array}$ & $\begin{array}{c}\text { Flux }^{3} \\
(17-60 \mathrm{keV})\end{array}$ & Type $^{4}$ & Notes \\
\hline N172 & MXB 0656-072 & 104.567 & -7.215 & $4.22 \pm 0.16$ & HMXB & \\
\hline N173 & IGR J07072-1227 & 106.810 & -12.460 & $0.78 \pm 0.14$ & UNIDENT & R 52; \\
\hline N174 & SWIFT J0710.5+5908 & 107.613 & 59.141 & $1.85 \pm 0.29$ & BLAZAR & $\mathrm{z}=0.125 ; \mathrm{B} 16$ \\
\hline N175 & IGR J07141+0146 & 108.540 & 1.770 & $1.28 \pm 0.24$ & UNIDENT & R 53; !B16; \\
\hline N176 & S5 $0716+714$ & 110.510 & 71.320 & $0.65 \pm 0.12$ & BLAZAR & $\mathrm{z}=0.300 ;$ !B 16 \\
\hline N177 & PKS 0723-008 & 111.480 & -0.926 & $1.12 \pm 0.22$ & BLAZAR & $\mathrm{z}=0.128$ \\
\hline N178 & IGR J07264-3553 & 111.611 & -35.883 & $1.18 \pm 0.28$ & SEYFERT & $\mathrm{z}=0.029 ; \mathrm{B} 16$ \\
\hline N179 & SWIFT J0728.8-2605 & 112.201 & -26.091 & $1.84 \pm 0.17$ & HMXB & \\
\hline N180 & IGR J07296-5854 & 112.413 & -58.905 & $0.96 \pm 0.22$ & UNIDENT & R 37; !B16; \\
\hline N181 & SWIFT J0732.5-1331 & 113.126 & -13.491 & $2.15 \pm 0.13$ & $\mathrm{CV}$ & \\
\hline N182 & IGR J07328-4640 & 113.214 & -46.682 & $0.72 \pm 0.16$ & BLAZAR? & PKS 0731-465; !B16; \\
\hline N183 & IGR J07396-3143 & 114.902 & -31.742 & $1.73 \pm 0.21$ & SEYFERT & R 22; z=0.026; !B16; \\
\hline N184 & MRK 79 & 115.637 & 49.810 & $4.26 \pm 0.68$ & SEYFERT & $\mathrm{z}=0.022$ \\
\hline N185 & IGR J07433-2544 & 115.840 & -25.762 & $1.26 \pm 0.15$ & SEYFERT & R 22; z=0.023; !B16; \\
\hline N186 & SWIFT J0747.9-7327 & 116.984 & -73.455 & $0.87 \pm 0.13$ & SEYFERT & $\mathrm{z}=0.036 ; \mathrm{B} 16$ \\
\hline N187 & EXO 0748-676 & 117.140 & -67.757 & $5.66 \pm 0.15$ & LMXB & \\
\hline N188 & IGR J07563-4137 & 119.074 & -41.637 & $0.90 \pm 0.11$ & SEYFERT & R 54,45; z=0.021; \\
\hline N189 & IGR J07563+5919 & 119.091 & 59.321 & $0.83 \pm 0.21$ & UNIDENT & R $37 ;$ !B 16 \\
\hline N190 & IGR J07597-3842 & 119.926 & -38.726 & $2.91 \pm 0.12$ & SEYFERT & R 55,46; z=0.040; \\
\hline N191 & IGR J08004-4309 & 120.115 & -43.155 & $0.52 \pm 0.10$ & $\mathrm{CV}$ & R 22,56; \\
\hline N192 & ESO 209-G012 & 120.500 & -49.752 & $1.69 \pm 0.11$ & SEYFERT & $\mathrm{z}=0.041$ \\
\hline N193 & MRK 1210 & 121.041 & 5.109 & $4.09 \pm 0.54$ & SEYFERT & $\mathrm{z}=0.014$ \\
\hline N194 & PG $0804+761$ & 122.814 & 76.047 & $0.92 \pm 0.09$ & SEYFERT & $\mathrm{z}=0.100$ \\
\hline N195 & IGR J08190-3835 & 124.759 & -38.583 & $0.44 \pm 0.10$ & SEYFERT & R $57 ; z=0.009$ \\
\hline N196 & RX J0818.9-2252 & 124.780 & -22.890 & $0.75 \pm 0.16$ & SEYFERT & $\mathrm{z}=0.035 ; ! \mathrm{B} 16$ \\
\hline N197 & IGR J08215-1320 & 125.390 & -13.340 & $0.99 \pm 0.14$ & SEYFERT & $\mathrm{R} 11 ; \mathrm{z}=0.015 ;$ !B16; \\
\hline N198 & SWIFT J0823.4-0457 & 125.752 & -4.913 & $1.80 \pm 0.23$ & SEYFERT & $\mathrm{z}=0.022 ; \mathrm{B} 16$ \\
\hline N199 & SWIFT J0826.2-7033 & 126.475 & -70.577 & $1.25 \pm 0.18$ & $\mathrm{CV}$ & !B 16 \\
\hline N200 & IGR J08297-4250 & 127.450 & -42.840 & $0.54 \pm 0.08$ & AGN & R 39; !B16; \\
\hline N201 & IGR J08321-1808 & 128.030 & -18.150 & $1.05 \pm 0.15$ & SEYFERT & R 53,11; z=0.135; !B16; \\
\hline N202 & Vela pulsar & 128.835 & -45.179 & $9.07 \pm 0.08$ & SNR / PULSAR & \\
\hline N203 & SWIFT J0835.5-0902 & 128.882 & -9.070 & $1.31 \pm 0.17$ & AGN? & !B16; \\
\hline N204 & NGC 2617 & 128.912 & -4.088 & $4.05 \pm 0.24$ & SEYFERT & $\mathrm{z}=0.014 ;$ !B16; \\
\hline N205 & GS 0834-43 & 128.979 & -43.185 & $0.93 \pm 0.08$ & HMXB & !B 16 \\
\hline N206 & 4U 0836-429 & 129.349 & -42.897 & $15.39 \pm 0.08$ & LMXB & \\
\hline N207 & FAIRALL 1146 & 129.634 & -35.986 & $1.79 \pm 0.11$ & SEYFERT & $\mathrm{z}=0.032$ \\
\hline N208 & IGR J08390-4833 & 129.681 & -48.521 & $0.76 \pm 0.08$ & $\mathrm{CV}$ & R 58,59,60; \\
\hline N209 & 3C 206 & 129.961 & -12.243 & $1.77 \pm 0.15$ & SEYFERT & $\mathrm{z}=0.198 ; \mathrm{B} 16$ \\
\hline $\mathrm{N} 210$ & S5 $0836+71$ & 130.353 & 70.905 & $4.47 \pm 0.08$ & BLAZAR & $\mathrm{z}=2.170$ \\
\hline $\mathrm{N} 211$ & IGR J08453-3529 & 131.341 & -35.488 & $0.66 \pm 0.12$ & SEYFERT & $\mathrm{R} 15 ; \mathrm{z}=0.137$ \\
\hline $\mathrm{N} 212$ & IGR J08503+6630 & 132.580 & 66.500 & $0.51 \pm 0.08$ & UNIDENT & R 37; !B16; \\
\hline $\mathrm{N} 213$ & NGC 2655 & 133.860 & 78.220 & $0.55 \pm 0.09$ & AGN & $\mathrm{z}=0.005 ; \mathrm{B} 16$ \\
\hline N214 & IGR J08557+6420 & 133.887 & 64.361 & $0.75 \pm 0.09$ & SEYFERT & R 15; z=0.036; \\
\hline $\mathrm{N} 215$ & Vela X-1 & 135.531 & -40.556 & $278.79 \pm 0.09$ & HMXB & \\
\hline $\mathrm{N} 216$ & IGR J09026-4812 & 135.650 & -48.229 & $1.61 \pm 0.08$ & SEYFERT & $\mathrm{R} 61 ; \mathrm{z}=0.039$ \\
\hline $\mathrm{N} 217$ & IGR J09025-6814 & 135.664 & -68.227 & $1.34 \pm 0.17$ & AGN & $\mathrm{R} 7 ; \mathrm{z}=0.013$ \\
\hline N218 & 1RXS J090431.1-382920 & 136.130 & -38.489 & $0.55 \pm 0.10$ & SEYFERT & $\mathrm{R} 47 ; \mathrm{z}=0.016$ \\
\hline N219 & IGR J09082-1336 & 137.070 & -13.610 & $0.89 \pm 0.18$ & UNIDENT & !B 16 \\
\hline $\mathrm{N} 220$ & ABELL 754 & 137.230 & -9.700 & $0.83 \pm 0.19$ & CLUSTER & $\mathrm{z}=0.054 ;$ !B16; \\
\hline $\mathrm{N} 221$ & SWIFT J0911.9-6452 & 138.010 & -64.868 & $5.07 \pm 0.13$ & LMXB & R 62; !B16; \\
\hline N222 & IRAS 09149-6206 & 139.036 & -62.333 & $1.80 \pm 0.12$ & SEYFERT & $\mathrm{z}=0.057$ \\
\hline $\mathrm{N} 223$ & IGR J09189-4418 & 139.731 & -44.312 & $0.36 \pm 0.09$ & SEYFERT? & R 63,16; \\
\hline $\mathrm{N} 224$ & 4U 0919-54 & 140.098 & -55.206 & $5.29 \pm 0.09$ & LMXB & \\
\hline $\mathrm{N} 225$ & SWIFT J0920.8-0805 & 140.190 & -8.050 & $3.09 \pm 0.22$ & SEYFERT & $\mathrm{z}=0.020$ \\
\hline N226 & PKS 0921-213 & 140.930 & -21.630 & $1.30 \pm 0.28$ & BLAZAR & $\mathrm{z}=0.052 ; \mathrm{B} 16$ \\
\hline $\mathrm{N} 227$ & MRK 110 & 141.276 & 52.296 & $4.79 \pm 0.22$ & SEYFERT & $\mathrm{z}=0.036$ \\
\hline N228 & IGR J09253+6929 & 141.439 & 69.470 & $0.88 \pm 0.07$ & SEYFERT & R 64,65,37; z=0.040; \\
\hline N229 & IGR J09278-3935 & 141.970 & -39.590 & $0.53 \pm 0.11$ & UNIDENT & R $53 ;$ !B 16 \\
\hline
\end{tabular}


Table A1 - continued from previous page

\begin{tabular}{|c|c|c|c|c|c|c|}
\hline Id & Name $^{1}$ & $\begin{array}{l}\mathrm{RA}^{2} \\
(\mathrm{deg})\end{array}$ & $\begin{array}{l}\operatorname{Dec}^{2} \\
(\mathrm{deg})\end{array}$ & $\begin{array}{c}\text { Flux }^{3} \\
(17-60 \mathrm{keV})\end{array}$ & Type $^{4}$ & Notes \\
\hline N230 & SWIFT J0929.7+6232 & 142.390 & 62.537 & $0.71 \pm 0.09$ & SEYFERT & $\mathrm{R} 47 ; \mathrm{z}=0.026$ \\
\hline N231 & IGR J09331-4725 & 143.280 & -47.430 & $0.50 \pm 0.09$ & UNIDENT & R 53; !B16; \\
\hline $\mathrm{N} 232$ & SWIFT J0935.9+6120 & 143.965 & 61.353 & $0.51 \pm 0.09$ & SEYFERT & $\mathrm{z}=0.039 ; \mathrm{B} 16$ \\
\hline $\mathrm{N} 233$ & NGC 2992 & 146.434 & -14.329 & $3.83 \pm 0.23$ & SEYFERT & $\mathrm{R} 66 ; \mathrm{z}=0.008$ \\
\hline $\mathrm{N} 234$ & MCG-05-23-16 & 146.922 & -30.947 & $11.87 \pm 0.29$ & SEYFERT & $\mathrm{z}=0.008$ \\
\hline $\mathrm{N} 235$ & SWIFT J0950.5+7318 & 147.496 & 73.253 & $0.57 \pm 0.07$ & SEYFERT & $\mathrm{R} 37 ; \mathrm{z}=0.058$ \\
\hline $\mathrm{N} 236$ & IGR J09522-6231 & 148.069 & -62.536 & $0.76 \pm 0.09$ & SEYFERT & $\mathrm{R} 58,57 ; \mathrm{z}=0.252$ \\
\hline $\mathrm{N} 237$ & M81 & 148.888 & 69.065 & $1.32 \pm 0.07$ & SEYFERT & \\
\hline $\mathrm{N} 238$ & M82 X-1 & 148.958 & 69.679 & $0.47 \pm 0.07$ & ULX & !B16; \\
\hline N239 & HolX X-1 & 149.472 & 69.063 & $0.27 \pm 0.07$ & ULX & \\
\hline $\mathrm{N} 240$ & SWIFT J0958.0-4208 & 149.487 & -42.133 & $1.55 \pm 0.14$ & CV? & R 67; \\
\hline $\mathrm{N} 241$ & SWIFT J0958.2-5732 & 149.650 & -57.450 & $0.42 \pm 0.08$ & UNIDENT & R 38; !B16; \\
\hline $\mathrm{N} 242$ & NGC 3081 & 149.870 & -22.819 & $4.72 \pm 0.29$ & SEYFERT & $\mathrm{z}=0.008$ \\
\hline N243 & NGC 3079 & 150.491 & 55.680 & $2.13 \pm 0.16$ & SEYFERT & $\mathrm{z}=0.004$ \\
\hline $\mathrm{N} 244$ & GRO J1008-57 & 152.419 & -58.282 & $7.19 \pm 0.08$ & HMXB & \\
\hline $\mathrm{N} 245$ & ESO 263-13 & 152.463 & -42.799 & $1.39 \pm 0.16$ & SEYFERT & $\mathrm{z}=0.033$ \\
\hline N246 & IGR J10100-5655 & 152.523 & -56.916 & $0.70 \pm 0.08$ & HMXB & R 68,46,69; \\
\hline $\mathrm{N} 247$ & IGR J10109-5746 & 152.774 & -57.797 & $1.53 \pm 0.08$ & $\mathrm{CV}$ & R 70,71; \\
\hline $\mathrm{N} 248$ & NGC 3227 & 155.873 & 19.870 & $8.50 \pm 0.44$ & SEYFERT & $\mathrm{z}=0.004$ \\
\hline $\mathrm{N} 249$ & IGR J10252+6716 & 156.310 & 67.310 & $0.48 \pm 0.07$ & SEYFERT & R 37; z=0.039; !B16; \\
\hline $\mathrm{N} 250$ & IGR J10257+4532 & 156.430 & 45.550 & $1.14 \pm 0.24$ & UNIDENT & !B 16 \\
\hline $\mathrm{N} 251$ & ESO $317-41$ & 157.850 & -42.090 & $0.90 \pm 0.20$ & AGN & $\mathrm{z}=0.020 ; \mathrm{B} 16$ \\
\hline $\mathrm{N} 252$ & NGC 3281 & 157.961 & -34.860 & $4.84 \pm 0.27$ & SEYFERT & $\mathrm{z}=0.011$ \\
\hline $\mathrm{N} 253$ & SWIFT J1033.6+7303 & 158.378 & 73.087 & $0.45 \pm 0.07$ & SEYFERT? & $\mathrm{R} 37,33 ; \mathrm{z}=0.022 ; \mathrm{B} 16$ \\
\hline $\mathrm{N} 254$ & $4 \mathrm{U} 1036-56$ & 159.454 & -56.745 & $1.18 \pm 0.07$ & HMXB & \\
\hline $\mathrm{N} 255$ & IGR J10380+8435 & 159.513 & 84.587 & $0.86 \pm 0.20$ & UNIDENT & R 37; !B16; \\
\hline $\mathrm{N} 256$ & IGR J10386-4947 & 159.672 & -49.790 & $1.33 \pm 0.10$ & SEYFERT & $\mathrm{R} 72 ; \mathrm{z}=0.060$ \\
\hline $\mathrm{N} 257$ & IGR J10404-4625 & 160.134 & -46.386 & $1.32 \pm 0.14$ & SEYFERT & R 54,$73 ; z=0.024$ \\
\hline $\mathrm{N} 258$ & SWIFT J1044.1+7024 & 161.061 & 70.355 & $0.69 \pm 0.07$ & SEYFERT & R 22; z=0.033; !B16; \\
\hline N259 & IGR J10447-6027 & 161.156 & -60.448 & $0.77 \pm 0.07$ & SEYFERT? & R 74,75,76,77; z=0.047; \\
\hline N260 & S5 $1039+81$ & 161.220 & 80.890 & $0.73 \pm 0.12$ & BLAZAR & R 37; z=1.260; !B16; \\
\hline N261 & ETA CAR & 161.221 & -59.707 & $0.72 \pm 0.07$ & STAR & \\
\hline N262 & IGR J10595-5125 & 164.880 & -51.420 & $0.45 \pm 0.09$ & SEYFERT & $\mathrm{z}=0.019 ;$ ESO 215-14; !B16; \\
\hline N263 & IGR J11014-6103 & 165.440 & -61.000 & $0.41 \pm 0.07$ & SNR / PULSAR & R 52; \\
\hline N264 & $4 C 72.16$ & 165.453 & 72.427 & $0.44 \pm 0.08$ & BLAZAR & $\mathrm{R} 37 ; \mathrm{z}=1.460 ;$ !B16; \\
\hline N265 & IGR J11030+7027 & 165.740 & 70.497 & $0.38 \pm 0.08$ & UNIDENT & R 37; !B 16; \\
\hline N266 & MRK 421 & 166.116 & 38.210 & $18.28 \pm 0.14$ & BLAZAR & $\mathrm{z}=0.031$ \\
\hline N267 & IGR J11054-1120 & 166.350 & -11.350 & $2.89 \pm 0.64$ & UNIDENT & !B16; \\
\hline N268 & SWIFT J1105.7+5854 & 166.423 & 58.914 & $1.04 \pm 0.15$ & SEYFERT? & R 37; !B16; \\
\hline N269 & NGC 3516 & 166.718 & 72.559 & $2.61 \pm 0.08$ & SEYFERT & $\mathrm{z}=0.009$ \\
\hline $\mathrm{N} 270$ & IGR J11079+7106 & 166.992 & 71.109 & $0.48 \pm 0.08$ & SEYFERT & $\mathrm{R} 78 ; \mathrm{z}=0.060 ;$ !B16; \\
\hline $\mathrm{N} 271$ & IGR J11131-5853 & 168.290 & -58.890 & $0.34 \pm 0.07$ & UNIDENT & !B 16 \\
\hline $\mathrm{N} 272$ & IGR J11176+1707 & 169.410 & 17.120 & $3.03 \pm 0.63$ & UNIDENT & !B16; \\
\hline N273 & IGR J11187-5438 & 169.592 & -54.662 & $0.79 \pm 0.08$ & LMXB? & R 79,69; \\
\hline $\mathrm{N} 274$ & 1A $1118-61$ & 170.238 & -61.917 & $2.82 \pm 0.07$ & HMXB & \\
\hline $\mathrm{N} 275$ & Cen X-3 & 170.313 & -60.624 & $63.85 \pm 0.07$ & HMXB & \\
\hline N276 & IGR J11215-5952 & 170.440 & -59.870 & $0.67 \pm 0.07$ & HMXB & R 80,81,82; \\
\hline $\mathrm{N} 277$ & PSR J1124-5916 & 171.130 & -59.240 & $0.35 \pm 0.07$ & SNR / PULSAR & !B16; \\
\hline N278 & IGR J11275-5319 & 171.890 & -53.330 & $0.79 \pm 0.09$ & UNIDENT & !B16; \\
\hline N279 & IGR J11299-6557 & 172.490 & -65.960 & $0.43 \pm 0.09$ & AGN? & R 11; \\
\hline $\mathrm{N} 280$ & IGR J11305-6256 & 172.779 & -62.948 & $1.56 \pm 0.08$ & HMXB & R 83,3,73; \\
\hline $\mathrm{N} 281$ & IGR J11361-6003 & 174.089 & -60.064 & $0.72 \pm 0.08$ & SEYFERT & $\mathrm{R} 1 ; \mathrm{z}=0.014$ \\
\hline $\mathrm{N} 282$ & RX J1136.5+6737 & 174.125 & 67.618 & $0.66 \pm 0.10$ & BLAZAR & R 37; z=0.134; !B16; \\
\hline $\mathrm{N} 283$ & NGC 3783 & 174.747 & -37.759 & $9.13 \pm 0.49$ & SEYFERT & $\mathrm{z}=0.010$ \\
\hline $\mathrm{N} 284$ & GT Mus & 174.875 & -65.398 & $0.42 \pm 0.08$ & STAR & \\
\hline $\mathrm{N} 285$ & DO Dra & 175.910 & 71.689 & $0.61 \pm 0.10$ & $\mathrm{CV}$ & !B16; \\
\hline N286 & IGR J11435-6109 & 175.996 & -61.123 & $4.08 \pm 0.08$ & HMXB & R 84,7,85,69; \\
\hline N287 & SWIFT J1144.1+3652 & 176.119 & 36.904 & $0.72 \pm 0.12$ & SEYFERT & R $22 ; z=0.038 ; ! B 16$ \\
\hline
\end{tabular}


Table A1 - continued from previous page

\begin{tabular}{|c|c|c|c|c|c|c|}
\hline Id & Name $^{1}$ & $\begin{array}{l}\mathrm{RA}^{2} \\
(\mathrm{deg})\end{array}$ & $\begin{array}{l}\mathrm{Dec}^{2} \\
(\mathrm{deg})\end{array}$ & $\begin{array}{c}\text { Flux }^{3} \\
(17-60 \mathrm{keV})\end{array}$ & Type $^{4}$ & Notes \\
\hline $\mathrm{N} 288$ & MCG+13-09-002 & 176.318 & 79.681 & $1.34 \pm 0.13$ & SEYFERT & R 37; z=0.015; \\
\hline N289 & HE 1143-1810 & 176.420 & -18.460 & $3.38 \pm 0.47$ & SEYFERT & $\mathrm{z}=0.033$ \\
\hline N290 & PKS 1143-696 & 176.509 & -69.943 & $0.90 \pm 0.11$ & BLAZAR & $\mathrm{z}=0.243$ \\
\hline N291 & 1E 1145.1-6141 & 176.863 & -61.971 & $23.90 \pm 0.08$ & HMXB & \\
\hline N292 & RX J1147.9+0902 & 176.979 & 9.041 & $0.57 \pm 0.10$ & SEYFERT & R 37; z=0.069; !B16; \\
\hline N293 & 4U $1145-619$ & 177.000 & -62.207 & $2.60 \pm 0.08$ & HMXB & \\
\hline N294 & IGR J11485+2936 & 177.130 & 29.610 & $0.78 \pm 0.18$ & SEYFERT & R 37; z=0.023; !B16; \\
\hline N295 & IGR J11523+0713 & 178.090 & 7.230 & $0.42 \pm 0.09$ & UNIDENT & !B16; \\
\hline N296 & 7C $1150+3324$ & 178.216 & 33.122 & $0.64 \pm 0.14$ & BLAZAR & R 37; z=1.394; !B16; \\
\hline N297 & SWIFT J1200.8+0650 & 180.242 & 6.809 & $1.16 \pm 0.08$ & SEYFERT & R $37 ; z=0.036$ \\
\hline N298 & MRK 1310 & 180.350 & -3.650 & $0.51 \pm 0.12$ & SEYFERT & R 37; $z=0.020 ;$ !B 16 \\
\hline N299 & IGR J12024-1127 & 180.622 & -11.460 & $1.09 \pm 0.24$ & UNIDENT & R 37; !B16; \\
\hline N300 & IGR J12026-5349 & 180.680 & -53.835 & $3.13 \pm 0.10$ & SEYFERT & R 70,45; z=0.028; \\
\hline N301 & NGC 4051 & 180.778 & 44.520 & $2.37 \pm 0.13$ & SEYFERT & $\mathrm{z}=0.002$ \\
\hline N302 & NGC 4074 & 181.110 & 20.291 & $0.94 \pm 0.13$ & SEYFERT & $\mathrm{z}=0.023$ \\
\hline N303 & $\mathrm{MCG}+05-29-005$ & 181.184 & 31.144 & $0.69 \pm 0.14$ & SEYFERT & $\mathrm{z}=0.025 ; \mathrm{B} 16$ \\
\hline N304 & NGC 4102 & 181.575 & 52.714 & $2.05 \pm 0.26$ & SEYFERT & R 86; z=0.003; !B16; \\
\hline N305 & B2 $1204+34$ & 181.887 & 33.878 & $0.95 \pm 0.12$ & SEYFERT & R 37; z=0.079; !B16; \\
\hline N306 & IGR J12086-6327 & 182.160 & -63.450 & $0.33 \pm 0.08$ & UNIDENT & R 53; !B16; \\
\hline N307 & MRK 198 & 182.316 & 47.087 & $1.24 \pm 0.15$ & SEYFERT & $\mathrm{z}=0.024$ \\
\hline N308 & NGC 4138 & 182.384 & 43.687 & $1.65 \pm 0.12$ & SEYFERT & $\mathrm{z}=0.003$ \\
\hline N309 & IGR J12095-0420 & 182.394 & -4.344 & $0.47 \pm 0.11$ & UNIDENT & R 37; !B16; \\
\hline N310 & NGC 4151 & 182.635 & 39.406 & $35.59 \pm 0.11$ & SEYFERT & $\mathrm{z}=0.003$ \\
\hline N311 & IGR J12107+3822 & 182.680 & 38.336 & $1.17 \pm 0.11$ & SEYFERT & R 87,15; z=0.023; \\
\hline N312 & IGR J12123-5802 & 183.108 & -58.006 & $0.63 \pm 0.09$ & CV & R 15 \\
\hline N313 & IGR J12131+0700 & 183.253 & 7.040 & $0.87 \pm 0.06$ & SEYFERT & R 1,37; z=0.210; \\
\hline N314 & $4 \mathrm{U} 1210-64$ & 183.255 & -64.880 & $0.57 \pm 0.09$ & HMXB & R 88; \\
\hline N315 & IGR J12134-6015 & 183.368 & -60.252 & $0.54 \pm 0.08$ & AGN? & R 89; \\
\hline N316 & Was 49 & 183.574 & 29.529 & $0.74 \pm 0.14$ & SEYFERT & $\mathrm{z}=0.064 ; \mathrm{B} 16$ \\
\hline N317 & IGR J12171+7047 & 184.288 & 70.797 & $0.62 \pm 0.13$ & SEYFERT & R 37,78; $z=0.007 ;$ !B 16; \\
\hline N318 & NGC 4235 & 184.293 & 7.198 & $2.84 \pm 0.06$ & SEYFERT & $\mathrm{z}=0.007$ \\
\hline N319 & NGC 4253 & 184.601 & 29.820 & $1.74 \pm 0.14$ & SEYFERT & $\mathrm{z}=0.013$ \\
\hline N320 & NGC 4258 & 184.721 & 47.290 & $1.17 \pm 0.15$ & SEYFERT & R 37; z=0.002; \\
\hline N321 & PKS 1217+02 & 185.050 & 2.062 & $0.59 \pm 0.07$ & SEYFERT & $\mathrm{z}=0.240$ \\
\hline N322 & IGR J12204+0452 & 185.120 & 4.868 & $0.56 \pm 0.06$ & UNIDENT & R 37; !B16; \\
\hline N323 & IGR J12208-0711 & 185.212 & -7.192 & $0.80 \pm 0.12$ & UNIDENT & R 37; !B16; \\
\hline N324 & 3A $1218+303$ & 185.331 & 30.169 & $0.85 \pm 0.13$ & BLAZAR & R 22; z=0.184; \\
\hline N325 & MRK 205 & 185.433 & 75.311 & $1.12 \pm 0.13$ & SEYFERT & $\mathrm{z}=0.071 ; \mathrm{B} 16$ \\
\hline N326 & PKS 1219+04 & 185.598 & 4.220 & $1.10 \pm 0.06$ & BLAZAR & $\mathrm{z}=0.966$ \\
\hline N327 & IGR J12224+0306 & 185.606 & 3.110 & $0.44 \pm 0.06$ & BLAZAR? & R 37; !B16; \\
\hline N328 & MRK 50 & 185.837 & 2.679 & $1.24 \pm 0.06$ & SEYFERT & $\mathrm{z}=0.024$ \\
\hline N329 & 4C 21.35 & 186.216 & 21.394 & $1.01 \pm 0.11$ & BLAZAR & $\mathrm{z}=0.434$ \\
\hline N330 & NGC 4388 & 186.447 & 12.664 & $12.32 \pm 0.07$ & SEYFERT & R 90; z=0.009; \\
\hline N331 & NGC 4395 & 186.463 & 33.556 & $1.50 \pm 0.12$ & SEYFERT & $\mathrm{z}=0.001$ \\
\hline N332 & GX 301-2 & 186.654 & -62.773 & $214.10 \pm 0.08$ & HMXB & \\
\hline N333 & XSS J12270-4859 & 187.000 & -48.893 & $1.60 \pm 0.15$ & LMXB & \\
\hline N334 & $3 \mathrm{C} 273$ & 187.277 & 2.054 & $16.64 \pm 0.06$ & BLAZAR & $\mathrm{z}=0.158$ \\
\hline N335 & IGR J12304+0946 & 187.620 & 9.776 & $0.27 \pm 0.06$ & UNIDENT & R 37; !B16; \\
\hline N336 & 1ES $1229+71.0$ & 187.902 & 70.737 & $0.71 \pm 0.15$ & SEYFERT & $\mathrm{z}=0.208 ; \mathrm{B} 16$ \\
\hline N337 & RT Cru & 188.721 & -64.566 & $5.16 \pm 0.09$ & $\mathrm{CV}$ & \\
\hline N338 & NGC 4507 & 188.903 & -39.905 & $11.04 \pm 0.18$ & SEYFERT & $\mathrm{z}=0.012$ \\
\hline N339 & IGR J12375+2156 & 189.392 & 21.944 & $0.55 \pm 0.11$ & UNIDENT & R 37; !B16; \\
\hline N340 & NGC 4579 & 189.432 & 11.818 & $0.65 \pm 0.06$ & SEYFERT & R 37; z=0.006; !B16; \\
\hline N341 & SWIFT J1238.6+0929 & 189.641 & 9.491 & $0.56 \pm 0.06$ & SEYFERT & R $47 ; z=0.083$ \\
\hline N342 & ESO 506-G027 & 189.728 & -27.315 & $4.36 \pm 0.38$ & SEYFERT & $\mathrm{z}=0.025$ \\
\hline N343 & XSS J12389-1614 & 189.775 & -16.182 & $2.29 \pm 0.22$ & SEYFERT & R 70,45; z=0.037; \\
\hline N344 & NGC 4593 & 189.910 & -5.347 & $4.71 \pm 0.10$ & SEYFERT & $\mathrm{z}=0.008$ \\
\hline \multirow[t]{2}{*}{ N345 } & SWIFT J1240.9+2735 & 190.229 & 27.531 & $0.70 \pm 0.13$ & SEYFERT & $\mathrm{R} 22,91 ; \mathrm{z}=0.057 ; \mathrm{B} 16$ \\
\hline & & & & & & Continued on next page \\
\hline
\end{tabular}


Table A1 - continued from previous page

\begin{tabular}{|c|c|c|c|c|c|c|}
\hline Id & Name $^{1}$ & $\begin{array}{l}\mathrm{RA}^{2} \\
(\mathrm{deg})\end{array}$ & $\begin{array}{l}\operatorname{Dec}^{2} \\
\text { (deg) }\end{array}$ & $\begin{array}{c}\text { Flux }^{3} \\
(17-60 \mathrm{keV})\end{array}$ & Type $^{4}$ & Notes \\
\hline N346 & IGR J12412+3007 & 190.334 & 30.122 & $0.83 \pm 0.12$ & AGN? & R 37,92; !B16; \\
\hline N347 & IGR J12415-5750 & 190.376 & -57.827 & $2.47 \pm 0.09$ & SEYFERT & R 7; z=0.024; \\
\hline N348 & IGR J12418+7805 & 190.650 & 78.122 & $0.76 \pm 0.16$ & SEYFERT & R 37; z=0.022; !B16; \\
\hline N349 & IGR J12480-5829 & 192.048 & -58.448 & $0.71 \pm 0.09$ & SEYFERT & R 14,15; z=0.028; \\
\hline $\mathrm{N} 350$ & IGR J12489-5930 & 192.240 & -59.500 & $0.53 \pm 0.09$ & UNIDENT & R 53; !B16; \\
\hline N351 & $4 \mathrm{U} 1246-588$ & 192.411 & -59.090 & $4.69 \pm 0.09$ & LMXB & \\
\hline N352 & NGC 4736 & 192.721 & 41.120 & $0.68 \pm 0.13$ & SEYFERT & R 37; z=0.001; !B16; \\
\hline N353 & NGC 4748 & 193.087 & -13.432 & $0.99 \pm 0.18$ & SEYFERT & $\mathrm{z}=0.014$ \\
\hline N354 & IGR J12529-6351 & 193.240 & -63.850 & $0.38 \pm 0.08$ & UNIDENT & R 53,93; !B16; \\
\hline N355 & ESO 323-G032 & 193.315 & -41.594 & $0.76 \pm 0.14$ & SEYFERT & R $22 ; z=0.016$ \\
\hline N356 & PKS 1252+11 & 193.659 & 11.685 & $0.47 \pm 0.07$ & BLAZAR & R 37; z=0.872; !B16; \\
\hline N357 & 3C 279 & 194.047 & -5.795 & $1.52 \pm 0.11$ & BLAZAR & $\mathrm{z}=0.536$ \\
\hline N358 & 4U 1254-69 & 194.419 & -69.289 & $2.41 \pm 0.10$ & LMXB & \\
\hline N359 & Coma & 194.880 & 27.951 & $1.50 \pm 0.12$ & CLUSTER & $\mathrm{z}=0.023 ; \mathrm{E}$ \\
\hline N360 & SWIFT J1300.1+1635 & 195.022 & 16.537 & $0.37 \pm 0.09$ & SEYFERT & $\mathrm{z}=0.080 ; \mathrm{B} 16$ \\
\hline N361 & $4 \mathrm{U} 1258-61$ & 195.322 & -61.602 & $19.00 \pm 0.08$ & HMXB & \\
\hline N362 & 1RXP J130159.6-635806 & 195.495 & -63.969 & $2.09 \pm 0.08$ & HMXB & R 94,69; \\
\hline N363 & PSR B1259-63 & 195.699 & -63.836 & $1.18 \pm 0.08$ & HMXB & R 95; \\
\hline N364 & MRK 783 & 195.700 & 16.376 & $1.20 \pm 0.09$ & SEYFERT & $\mathrm{z}=0.067$ \\
\hline N365 & IGR J13038+5348 & 195.997 & 53.792 & $0.92 \pm 0.16$ & SEYFERT & R 36,96; z=0.030; \\
\hline N366 & NGC 4941 & 196.015 & -5.545 & $0.73 \pm 0.12$ & SEYFERT & $\mathrm{z}=0.004$ \\
\hline N367 & NGC 4939 & 196.059 & -10.328 & $1.07 \pm 0.16$ & SEYFERT & $\mathrm{z}=0.010$ \\
\hline N368 & IGR J13045-5630 & 196.110 & -56.600 & $0.74 \pm 0.09$ & UNIDENT & \\
\hline N369 & NGC 4945 & 196.363 & -49.466 & $15.47 \pm 0.12$ & SEYFERT & $\mathrm{z}=0.002$ \\
\hline N370 & ESO 323-G077 & 196.620 & -40.422 & $2.56 \pm 0.12$ & SEYFERT & $\mathrm{z}=0.015$ \\
\hline N371 & IGR J13091+1137 & 197.283 & 11.629 & $2.72 \pm 0.09$ & SEYFERT & R 70,45; z=0.025; \\
\hline N372 & IGR J13107-5626 & 197.655 & -56.449 & $0.70 \pm 0.09$ & AGN? & R 97; \\
\hline N373 & IGR J13107-5551 & 197.697 & -55.876 & $1.55 \pm 0.09$ & SEYFERT & R 70,1; z=0.104; \\
\hline N374 & PG $1310-108$ & 198.274 & -11.128 & $0.83 \pm 0.19$ & SEYFERT & $\mathrm{z}=0.034$ \\
\hline N375 & NGC 5033 & 198.364 & 36.594 & $0.80 \pm 0.16$ & SEYFERT & $\mathrm{z}=0.003 ; ! \mathrm{B} 16$ \\
\hline N376 & IGR J13149+4422 & 198.787 & 44.419 & $1.25 \pm 0.16$ & SEYFERT & R 98,25; z=0.036; \\
\hline N377 & IGR J13168-7157 & 199.196 & -71.945 & $0.75 \pm 0.12$ & SEYFERT & R 99,15; z=0.070; \\
\hline N378 & RX J1317.0+3735 & 199.262 & 37.592 & $0.83 \pm 0.17$ & SEYFERT & $\mathrm{z}=0.195 ;$ !B 16 \\
\hline N379 & IGR J13186-6257 & 199.636 & -62.954 & $0.95 \pm 0.08$ & HMXB & R 100,101,76; \\
\hline N380 & SWIFT J1321.2+0859 & 200.241 & 8.950 & $0.75 \pm 0.10$ & AGN & R 22,102; z=0.033; \\
\hline N381 & MCG-03-34-064 & 200.611 & -16.733 & $1.62 \pm 0.30$ & SEYFERT & $\mathrm{z}=0.017$ \\
\hline N382 & SAX J1324.5-6313 & 201.161 & -63.228 & $0.43 \pm 0.08$ & LMXB & R 103; \\
\hline N383 & Cen A & 201.362 & -43.019 & $57.38 \pm 0.11$ & SEYFERT & $\mathrm{z}=0.002$ \\
\hline N384 & 4U 1323-62 & 201.653 & -62.135 & $14.15 \pm 0.08$ & LMXB & \\
\hline N385 & ABELL 1736 & 202.072 & -27.293 & $1.22 \pm 0.21$ & CLUSTER & $\mathrm{z}=0.046 ;$ !B16; \\
\hline N386 & IGR J13290-6323 & 202.268 & -63.392 & $0.40 \pm 0.08$ & UNIDENT & \\
\hline N387 & IGR J13292-4332 & 202.320 & -43.550 & $0.54 \pm 0.11$ & UNIDENT & !B16; \\
\hline N388 & ESO 509- G038 & 202.722 & -25.412 & $1.37 \pm 0.24$ & SEYFERT & $\mathrm{z}=0.026 ;$ !B16; \\
\hline N389 & IGR J13310-1355 & 202.756 & -13.931 & $1.43 \pm 0.31$ & UNIDENT & R 37; !B16; \\
\hline N390 & ESO 383-G018 & 203.362 & -34.028 & $1.41 \pm 0.14$ & SEYFERT & $\mathrm{z}=0.013$ \\
\hline N391 & MCG-6-30-15 & 203.982 & -34.297 & $2.37 \pm 0.14$ & SEYFERT & $\mathrm{z}=0.008$ \\
\hline N392 & NGC 5252 & 204.570 & 4.548 & $6.10 \pm 0.14$ & SEYFERT & $\mathrm{z}=0.023$ \\
\hline N393 & IGR J13402-6428 & 205.080 & -64.480 & $0.35 \pm 0.08$ & UNIDENT & R 52; \\
\hline N394 & MRK 268 & 205.297 & 30.378 & $1.63 \pm 0.16$ & SEYFERT & $\mathrm{z}=0.040$ \\
\hline N395 & NGC 5273 & 205.555 & 35.712 & $1.45 \pm 0.20$ & SEYFERT & R 22; z=0.003; !B16; \\
\hline N396 & IGR J13466+1921 & 206.676 & 19.385 & $0.66 \pm 0.16$ & SEYFERT & $\mathrm{z}=0.084$ \\
\hline N397 & $4 \mathrm{U} 1344-60$ & 206.897 & -60.615 & $6.68 \pm 0.08$ & SEYFERT & $\mathrm{z}=0.013$ \\
\hline N398 & IGR J13486+1554 & 207.168 & 15.901 & $0.72 \pm 0.15$ & UNIDENT & R 37; !B16; \\
\hline N399 & IC $4329 A$ & 207.332 & -30.312 & $15.34 \pm 0.17$ & SEYFERT & $\mathrm{z}=0.016$ \\
\hline N400 & TOL $1351-375$ & 208.561 & -37.775 & $0.94 \pm 0.11$ & SEYFERT & $\mathrm{z}=0.052$ \\
\hline N401 & IGR J13545-5958 & 208.626 & -59.963 & $0.41 \pm 0.08$ & UNIDENT & R 53; !B16; \\
\hline N402 & Ginga 1354-64 & 209.541 & -64.735 & $3.16 \pm 0.08$ & LMXB & !B16; \\
\hline N403 & IGR J14003-6326 & 210.142 & -63.428 & $1.20 \pm 0.08$ & SNR / PULSAR & R 104,105; \\
\hline
\end{tabular}


Table A1 - continued from previous page

\begin{tabular}{|c|c|c|c|c|c|c|}
\hline Id & Name $^{1}$ & $\begin{array}{l}\mathrm{RA}^{2} \\
(\mathrm{deg})\end{array}$ & $\begin{array}{l}\text { Dec }^{2} \\
(\mathrm{deg})\end{array}$ & $\begin{array}{c}\text { Flux }^{3} \\
(17-60 \mathrm{keV})\end{array}$ & Type $^{4}$ & Notes \\
\hline N404 & IGR J14044-6146 & 211.120 & -61.780 & $0.61 \pm 0.08$ & UNIDENT & R 53,52,106; \\
\hline N405 & IGR J14080-3023 & 212.010 & -30.391 & $1.06 \pm 0.16$ & SEYFERT & $\mathrm{R} 22 ; \mathrm{z}=0.025$ \\
\hline N406 & V834 Cen & 212.262 & -45.269 & $0.59 \pm 0.11$ & $\mathrm{CV}$ & \\
\hline N407 & IGR J14091-6108 & 212.280 & -61.140 & $0.49 \pm 0.08$ & $\mathrm{CV}$ & R 107; !B16; \\
\hline N408 & SWIFT J1410.9-4229 & 212.640 & -42.459 & $0.43 \pm 0.10$ & SEYFERT & R 22; $\mathrm{z}=0.035$ \\
\hline N409 & Circinus galaxy & 213.291 & -65.341 & $17.93 \pm 0.09$ & SEYFERT & $\mathrm{z}=0.001$ \\
\hline N410 & NGC 5506 & 213.305 & -3.210 & $12.74 \pm 0.21$ & SEYFERT & $\mathrm{z}=0.006$ \\
\hline N411 & PKS 1413-36 & 214.180 & -36.650 & $0.60 \pm 0.11$ & AGN & $\mathrm{z}=0.075 ; \mathrm{B} 16$ \\
\hline $\mathrm{N} 412$ & IGR J14175-4641 & 214.266 & -46.681 & $1.34 \pm 0.11$ & SEYFERT & R 70,46; z=0.076; \\
\hline N413 & NGC 5548 & 214.507 & 25.139 & $6.01 \pm 0.18$ & SEYFERT & $\mathrm{z}=0.017$ \\
\hline N414 & AX J1418.7-6058 & 214.701 & -60.915 & $0.42 \pm 0.08$ & SNR / PULSAR & R 22; \\
\hline N415 & ESO 511-G030 & 214.834 & -26.648 & $2.15 \pm 0.20$ & SEYFERT & $\mathrm{z}=0.023$ \\
\hline N416 & 4U 1416-62 & 215.285 & -62.680 & $2.04 \pm 0.08$ & HMXB & \\
\hline N417 & 3 A $1422+481$ & 215.388 & 47.741 & $0.95 \pm 0.14$ & SEYFERT & $\mathrm{z}=0.072$ \\
\hline N418 & IGR J14257-6117 & 216.430 & -61.290 & $0.61 \pm 0.08$ & $\mathrm{CV}$ & R 67,108; \\
\hline N419 & H $1426+428$ & 217.072 & 42.664 & $1.12 \pm 0.19$ & BLAZAR & $\mathrm{z}=0.129$ \\
\hline $\mathrm{N} 420$ & MRK 1383 & 217.278 & 1.280 & $0.90 \pm 0.19$ & SEYFERT & $\mathrm{z}=0.087 ; \mathrm{B} 16$ \\
\hline N421 & IGR J14298-6715 & 217.440 & -67.239 & $0.98 \pm 0.09$ & LMXB & R 104,1 \\
\hline N422 & IGR J14300+0118 & 217.510 & 1.310 & $1.00 \pm 0.19$ & UNIDENT & !B16; C; \\
\hline N423 & NGC 5643 & 218.172 & -44.209 & $1.18 \pm 0.10$ & SEYFERT & $\mathrm{z}=0.004$ \\
\hline N424 & IGR J14331-6112 & 218.270 & -61.260 & $0.54 \pm 0.08$ & HMXB & R 104,1,69; \\
\hline $\mathrm{N} 425$ & NGC 5674 & 218.468 & 5.458 & $1.17 \pm 0.18$ & SEYFERT & $\mathrm{z}=0.025$ \\
\hline N426 & MRK 817 & 219.092 & 58.794 & $1.41 \pm 0.15$ & SEYFERT & $\mathrm{z}=0.031 ; \mathrm{B} 16$ \\
\hline N427 & HE 1434-1600 & 219.155 & -16.192 & $2.31 \pm 0.26$ & SEYFERT & $\mathrm{z}=0.145$ \\
\hline N428 & MRK 477 & 220.156 & 53.510 & $1.03 \pm 0.14$ & SEYFERT & $\mathrm{z}=0.038 ; \mathrm{B} 16$ \\
\hline N429 & IGR J14417-5533 & 220.430 & -55.550 & $0.72 \pm 0.08$ & UNIDENT & R 53; !B16; \\
\hline N430 & NGC 5728 & 220.588 & -17.246 & $5.07 \pm 0.26$ & SEYFERT & $\mathrm{z}=0.009$ \\
\hline N431 & IGR J14471-6414 & 221.582 & -64.276 & $0.77 \pm 0.09$ & SEYFERT & R 104,$1 ; z=0.053$ \\
\hline N432 & IGR J14471-6319 & 221.789 & -63.288 & $0.55 \pm 0.08$ & SEYFERT & R 70,46; z=0.038; \\
\hline N433 & IGR J14488-4009 & 222.199 & -40.148 & $1.01 \pm 0.10$ & SEYFERT & R 67,109; z=0.123; \\
\hline N434 & IGR J14493-5534 & 222.313 & -55.596 & $1.56 \pm 0.08$ & AGN & R 58; z=0.002; \\
\hline N435 & IGR J14488-5942 & 222.320 & -59.760 & $0.87 \pm 0.08$ & HMXB & R 69; \\
\hline N436 & IGR J14515-5542 & 222.893 & -55.681 & $1.58 \pm 0.08$ & SEYFERT & $\mathrm{R} 68,46,3 ; \mathrm{z}=0.018$ \\
\hline N437 & IGR J14536-5522 & 223.413 & -55.351 & $1.12 \pm 0.08$ & $\mathrm{CV}$ & R 68,110; \\
\hline N438 & IGR J14552-5133 & 223.849 & -51.586 & $1.18 \pm 0.09$ & SEYFERT & R 70,46; z=0.016; \\
\hline N439 & IGR J14557-5448 & 223.960 & -54.810 & $0.47 \pm 0.08$ & UNIDENT & \\
\hline N440 & IGR J14561-3738 & 224.035 & -37.640 & $0.63 \pm 0.10$ & SEYFERT & R 58; z=0.024; \\
\hline N441 & IC $4518 \mathrm{~A}$ & 224.419 & -43.129 & $1.50 \pm 0.10$ & SEYFERT & $\mathrm{z}=0.016$ \\
\hline N442 & IGR J15038-6020 & 225.960 & -60.340 & $0.75 \pm 0.08$ & $\mathrm{CV}$ & R 111; \\
\hline N443 & MRK 841 & 225.992 & 10.435 & $1.96 \pm 0.24$ & SEYFERT & $\mathrm{z}=0.036$ \\
\hline N444 & SWIFT J1508.6-4953 & 227.163 & -49.869 & $0.92 \pm 0.09$ & BLAZAR & R 112; \\
\hline N445 & IGR J15094-6649 & 227.363 & -66.827 & $1.72 \pm 0.10$ & CV & R 70,110; \\
\hline N446 & IRAS 15091-2107 & 227.972 & -21.371 & $1.82 \pm 0.22$ & SEYFERT & $\mathrm{z}=0.045$ \\
\hline N447 & PKS 1510-08 & 228.210 & -9.100 & $2.25 \pm 0.18$ & BLAZAR & $z=0.360$ \\
\hline N448 & PSR $1509-58$ & 228.483 & -59.143 & $12.17 \pm 0.08$ & SNR / PULSAR & \\
\hline N449 & SWIFT J1513.8-8125 & 228.685 & -81.386 & $1.34 \pm 0.22$ & SEYFERT & R 113,15; z=0.068; \\
\hline N450 & 4U $1516-569$ & 230.172 & -57.167 & $5.20 \pm 0.08$ & HMXB & \\
\hline N451 & IGR J15299-2355 & 232.480 & -23.920 & $1.05 \pm 0.20$ & UNIDENT & !B16; \\
\hline $\mathrm{N} 452$ & IGR J15301-3840 & 232.540 & -38.670 & $0.90 \pm 0.10$ & AGN & $\mathrm{z}=0.016$ \\
\hline N453 & MCG-01-40-001 & 233.326 & -8.724 & $1.83 \pm 0.17$ & SEYFERT & $\mathrm{z}=0.023$ \\
\hline N454 & IGR J15335-5420 & 233.390 & -54.340 & $0.52 \pm 0.08$ & UNIDENT & R 114; !B16; T; \\
\hline N455 & IGR J15360-5750 & 234.015 & -57.811 & $1.16 \pm 0.08$ & AGN & R 70,115; z=0.023; \\
\hline N456 & SWIFT J1539.2-6227 & 234.760 & -62.430 & $0.40 \pm 0.09$ & LMXB & R 116,117; T; \\
\hline N457 & IGR J15414-5030 & 235.369 & -50.502 & $0.62 \pm 0.08$ & SEYFERT & R $100 ; z=0.032$ \\
\hline N458 & 4U $1538-522$ & 235.596 & -52.385 & $21.09 \pm 0.08$ & НMXB & \\
\hline N459 & 4U 1543-624 & 236.956 & -62.566 & $3.82 \pm 0.10$ & LMXB & \\
\hline N460 & NY Lup & 237.057 & -45.480 & $5.97 \pm 0.08$ & $\mathrm{CV}$ & \\
\hline N461 & NGC 5995 & 237.089 & -13.738 & $2.83 \pm 0.13$ & SEYFERT & $\mathrm{z}=0.025$ \\
\hline
\end{tabular}


Table A1 - continued from previous page

\begin{tabular}{|c|c|c|c|c|c|c|}
\hline Id & Name $^{1}$ & $\begin{array}{l}\mathrm{RA}^{2} \\
(\mathrm{deg})\end{array}$ & $\begin{array}{l}\operatorname{Dec}^{2} \\
(\mathrm{deg})\end{array}$ & $\begin{array}{c}\text { Flux }^{3} \\
(17-60 \mathrm{keV})\end{array}$ & Type $^{4}$ & Notes \\
\hline N462 & 1E 1547.0-5408 & 237.725 & -54.306 & $1.22 \pm 0.08$ & MAGNETAR & \\
\hline N463 & XTE J1550-564 & 237.757 & -56.458 & $13.83 \pm 0.08$ & LMXB & $\mathrm{T}$ \\
\hline N464 & IGR J15539-6142 & 238.438 & -61.683 & $0.74 \pm 0.10$ & SEYFERT & R 104,118; z=0.015; \\
\hline N465 & IGR J15549-3740 & 238.713 & -37.665 & $0.65 \pm 0.11$ & SEYFERT & $\mathrm{R} 15 ; \mathrm{z}=0.019$ \\
\hline N466 & IGR J15550-4306 & 238.760 & -43.110 & $0.56 \pm 0.08$ & UNIDENT & R 53; !B16; \\
\hline N467 & 2S $1553-542$ & 239.454 & -54.415 & $0.37 \pm 0.08$ & HMXB & \\
\hline N468 & $\mathrm{T} \mathrm{CrB}$ & 239.813 & 25.912 & $1.38 \pm 0.30$ & $\mathrm{CV}$ & \\
\hline N469 & IGR J16005-4645 & 240.130 & -46.760 & $0.37 \pm 0.07$ & AGN? & R 119; !B16; \\
\hline N470 & $4 \mathrm{U} 1556-605$ & 240.336 & -60.693 & $0.50 \pm 0.09$ & LMXB & \\
\hline N471 & IGR J16058-7253 & 241.477 & -72.901 & $1.24 \pm 0.17$ & SEYFERT & R 120; z=0.090; AGN pair; \\
\hline N472 & IGR J16113-4200 & 242.827 & -42.015 & $0.41 \pm 0.08$ & UNIDENT & !B16; \\
\hline N473 & IGR J16120-3543 & 242.974 & -35.754 & $1.76 \pm 0.11$ & AGN? & R 111; \\
\hline N474 & WKK 6092 & 242.985 & -60.624 & $1.47 \pm 0.10$ & SEYFERT & $\mathrm{z}=0.016$ \\
\hline N475 & $4 \mathrm{U} 1608-52$ & 243.177 & -52.422 & $20.97 \pm 0.07$ & LMXB & \\
\hline N476 & IGR J16138+1043 & 243.470 & 10.720 & $1.53 \pm 0.34$ & UNIDENT & !B16; \\
\hline N477 & AT2018cow & 244.000 & 22.270 & $1.58 \pm 0.30$ & SUPERNOVA & $\mathrm{R} 121 ; \mathrm{z}=0.014 ;$ !B16; T; \\
\hline N478 & IGR J16167-4957 & 244.145 & -49.981 & $1.66 \pm 0.07$ & $\mathrm{CV}$ & R 122,123 \\
\hline N479 & IGR J16173-5023 & 244.353 & -50.363 & $0.52 \pm 0.07$ & UNIDENT & \\
\hline N480 & PSR J1617-5055 & 244.364 & -50.939 & $0.63 \pm 0.07$ & SNR / PULSAR & \\
\hline N481 & 3C 332.0 & 244.427 & 32.376 & $0.91 \pm 0.17$ & SEYFERT & $\mathrm{z}=0.151 ;$ !B16; \\
\hline N482 & IGR J16181-5407 & 244.530 & -54.120 & $0.46 \pm 0.08$ & AGN & R 77,111; \\
\hline N483 & IGR J16185-5928 & 244.624 & -59.459 & $1.17 \pm 0.09$ & SEYFERT & R 70,46; z=0.035; \\
\hline N484 & IGR J16195-2807 & 244.878 & -28.146 & $2.66 \pm 0.16$ & LMXB & R 54,124; \\
\hline N485 & IGR J16195-4945 & 244.891 & -49.743 & $2.05 \pm 0.07$ & HMXB & R 122,125; \\
\hline N486 & Sco X-1 & 244.980 & -15.641 & $861.29 \pm 0.11$ & LMXB & \\
\hline N487 & MAXI J1621-501 & 245.092 & -50.020 & $0.97 \pm 0.07$ & LMXB & !B16; T; \\
\hline N488 & IGR J16207-5129 & 245.187 & -51.508 & $3.68 \pm 0.07$ & HMXB & R 122,125; \\
\hline N489 & IGR J16267-3303 & 246.700 & -33.060 & $0.53 \pm 0.12$ & UNIDENT & !B 16 \\
\hline N490 & $4 \mathrm{U} 1624-49$ & 247.004 & -49.206 & $3.79 \pm 0.07$ & LMXB & \\
\hline N491 & IGR J16283-4838 & 247.068 & -48.654 & $0.60 \pm 0.07$ & HMXB & R 126,127; \\
\hline N492 & IGR J16287-5021 & 247.120 & -50.369 & $0.57 \pm 0.07$ & LMXB & R 15 \\
\hline N493 & IGR J16293-4603 & 247.311 & -46.076 & $0.44 \pm 0.07$ & LMXB & R 128,129; \\
\hline N494 & IRAS $16288+3929$ & 247.679 & 39.379 & $0.79 \pm 0.14$ & SEYFERT & $\mathrm{z}=0.031$ \\
\hline N495 & MAXI J1631-479 & 247.809 & -47.807 & $12.36 \pm 0.07$ & LMXB? & R 130; C; \\
\hline N496 & IGR J16318-4848 & 247.951 & -48.816 & $30.69 \pm 0.07$ & HMXB & R 131,132; \\
\hline N497 & IGR J16320-4751 & 248.008 & -47.876 & $20.99 \pm 0.07$ & HMXB & R 133,134,135,136,137; C; \\
\hline N498 & 4U $1626-67$ & 248.073 & -67.465 & $25.78 \pm 0.16$ & LMXB & \\
\hline N499 & 4U 1630-47 & 248.524 & -47.390 & $19.02 \pm 0.07$ & LMXB & \\
\hline N500 & ESO 137-G34 & 248.807 & -58.089 & $1.54 \pm 0.09$ & SEYFERT & $\mathrm{z}=0.009$ \\
\hline N501 & IGR J16358-4726 & 248.992 & -47.407 & $0.60 \pm 0.07$ & LMXB & R 138,139; \\
\hline N502 & Triangulum A & 249.554 & -64.368 & $1.22 \pm 0.14$ & CLUSTER & $\mathrm{z}=0.051$ \\
\hline N503 & IGR J16385-2057 & 249.643 & -20.914 & $0.91 \pm 0.12$ & SEYFERT & R $1 ; z=0.027$ \\
\hline N504 & AX J163904-4642 & 249.773 & -46.704 & $5.85 \pm 0.07$ & HMXB & R 140 \\
\hline N505 & $4 \mathrm{U} 1636-53$ & 250.230 & -53.754 & $34.86 \pm 0.08$ & LMXB & \\
\hline N506 & IGR J16413-4046 & 250.331 & -40.794 & $0.57 \pm 0.07$ & AGN? & R 16; \\
\hline N507 & IGR J16418-4532 & 250.455 & -45.538 & $5.03 \pm 0.07$ & HMXB & R 141,135; \\
\hline N508 & GX $340+0$ & 251.449 & -45.612 & $33.16 \pm 0.07$ & LMXB & \\
\hline N509 & IGR J16459-2325 & 251.480 & -23.430 & $1.28 \pm 0.10$ & UNIDENT & R 53; !B16; \\
\hline N510 & IGR J16465-4507 & 251.648 & -45.118 & $1.60 \pm 0.07$ & HMXB & R 142,143; \\
\hline N511 & IGR J16479-4514 & 252.020 & -45.213 & $5.13 \pm 0.07$ & HMXB & R $144,145,135$ \\
\hline N512 & IGR J16482-3036 & 252.047 & -30.580 & $1.31 \pm 0.08$ & SEYFERT & R 54,73; z=0.031; \\
\hline N513 & IGR J16494-1740 & 252.350 & -17.680 & $0.79 \pm 0.11$ & SEYFERT & $\mathrm{R} 11 ; \mathrm{z}=0.023 ;$ !B16; \\
\hline N514 & IGR J16493-4348 & 252.373 & -43.823 & $2.57 \pm 0.07$ & HMXB & R $146,147,148$ \\
\hline N515 & IGR J16500-3307 & 252.481 & -33.110 & $1.47 \pm 0.07$ & $\mathrm{CV}$ & R 54,1; \\
\hline N516 & NGC 6230 & 252.660 & 4.570 & $0.99 \pm 0.23$ & SEYFERT & R 149; z=0.032; \\
\hline N517 & NGC 6221 & 253.080 & -59.219 & $1.75 \pm 0.11$ & SEYFERT & $\mathrm{z}=0.005 ; \mathrm{C}$ \\
\hline N518 & XTE J1652-453 & 253.085 & -45.344 & $1.32 \pm 0.07$ & LMXB? & R 150; T; \\
\hline N519 & NGC 6240 & 253.245 & 2.389 & $4.00 \pm 0.21$ & SEYFERT & $\mathrm{z}=0.025$ \\
\hline
\end{tabular}


Table A1 - continued from previous page

\begin{tabular}{|c|c|c|c|c|c|c|}
\hline Id & Name $^{1}$ & $\begin{array}{l}\mathrm{RA}^{2} \\
(\mathrm{deg})\end{array}$ & $\begin{array}{l}\operatorname{Dec}^{2} \\
(\mathrm{deg})\end{array}$ & $\begin{array}{c}\text { Flux }^{3} \\
(17-60 \mathrm{keV})\end{array}$ & Type $^{4}$ & Notes \\
\hline N520 & MRK 501 & 253.473 & 39.758 & $5.42 \pm 0.13$ & BLAZAR & $\mathrm{z}=0.034$ \\
\hline N521 & GRO J1655-40 & 253.499 & -39.844 & $6.60 \pm 0.07$ & LMXB & \\
\hline N522 & IGR J16547-1916 & 253.685 & -19.275 & $1.19 \pm 0.10$ & $\mathrm{CV}$ & R 15,$151 ;$ \\
\hline N523 & IGR J16560-4958 & 253.989 & -49.967 & $0.58 \pm 0.07$ & AGN & R 16 \\
\hline N524 & IGR J16558-5203 & 254.031 & -52.077 & $2.16 \pm 0.08$ & SEYFERT & R 122,$46 ; z=0.054$ \\
\hline N525 & IGR J16562-3301 & 254.085 & -33.042 & $1.66 \pm 0.07$ & BLAZAR & R $152 ; z=2.400$ \\
\hline N526 & Her X-1 & 254.459 & 35.343 & $124.15 \pm 0.12$ & LMXB & \\
\hline N527 & IGR J16580-3247 & 254.520 & -32.800 & $0.31 \pm 0.06$ & UNIDENT & !B16; \\
\hline N528 & SWIFT J1658.2-4242 & 254.553 & -42.698 & $1.76 \pm 0.07$ & LMXB? & R 153,154; !B16; T; \\
\hline N529 & MAXI J1659-152 & 254.792 & -15.268 & $10.09 \pm 0.12$ & LMXB & R 155; T; \\
\hline N530 & AX J1700.2-4220 & 255.078 & -42.347 & $1.72 \pm 0.07$ & HMXB & R 110; \\
\hline N531 & OAO 1657-415 & 255.203 & -41.655 & $76.39 \pm 0.07$ & HMXB & \\
\hline N532 & XTE J1701-462 & 255.241 & -46.188 & $2.18 \pm 0.07$ & LMXB & $\mathrm{T}$ \\
\hline N533 & IGR J17009+3559 & 255.246 & 35.982 & $1.20 \pm 0.12$ & AGN & R 156,$15 ; z=0.113 ;$ \\
\hline N534 & IGR J17014-4306 & 255.360 & -43.110 & $0.43 \pm 0.07$ & $\mathrm{CV}$ & \\
\hline N535 & XTE J1701-407 & 255.435 & -40.858 & $5.11 \pm 0.07$ & LMXB & \\
\hline N536 & MXB 1659-298 & 255.500 & -29.960 & $0.28 \pm 0.06$ & LMXB & R 157; !B16; \\
\hline N537 & GX 339-4 & 255.705 & -48.791 & $77.25 \pm 0.07$ & LMXB & \\
\hline N538 & IGR J17036+3734 & 255.892 & 37.567 & $0.52 \pm 0.12$ & SEYFERT & R 57; z=0.065; !B16; \\
\hline N539 & $4 \mathrm{U} 1700-37$ & 255.982 & -37.842 & $243.43 \pm 0.06$ & HMXB & \\
\hline N540 & IGR J17040-4305 & 256.010 & -43.080 & $0.36 \pm 0.07$ & $\mathrm{CV} ?$ & R 93; !B16; \\
\hline N541 & GX $349+2$ & 256.432 & -36.422 & $42.50 \pm 0.06$ & LMXB & \\
\hline N542 & 4U $1702-429$ & 256.564 & -43.037 & $18.66 \pm 0.07$ & LMXB & \\
\hline N543 & IGR J17062-6143 & 256.579 & -61.698 & $2.26 \pm 0.15$ & LMXB & R 158,159,159; \\
\hline N544 & 1RXS J170849.0-400910 & 257.198 & -40.154 & $1.49 \pm 0.06$ & MAGNETAR & \\
\hline N545 & $4 \mathrm{U} 1705-32$ & 257.225 & -32.320 & $1.28 \pm 0.06$ & LMXB & \\
\hline N546 & 4U $1705-44$ & 257.227 & -44.101 & $19.61 \pm 0.07$ & LMXB & \\
\hline N547 & IGR J17091-3624 & 257.289 & -36.405 & $9.75 \pm 0.06$ & LMXB & R 160,161,162,136; \\
\hline N548 & XTE J1709-267 & 257.388 & -26.656 & $1.18 \pm 0.06$ & LMXB & \\
\hline N549 & IGR J17098-2344 & 257.450 & -23.750 & $0.54 \pm 0.06$ & SEYFERT & $\mathrm{R} 11 ; \mathrm{z}=0.036 ;$ !B16; \\
\hline N550 & IGR J17098-3628 & 257.485 & -36.448 & $5.15 \pm 0.06$ & LMXB & R 163,164; \\
\hline N551 & XTE J1710-281 & 257.550 & -28.126 & $3.28 \pm 0.06$ & LMXB & \\
\hline N552 & RX J1713.7-3946A & 257.940 & -39.543 & $0.61 \pm 0.06$ & SNR & R 165; !B16; E; \\
\hline N553 & IGR J17118-3155 & 257.959 & -31.927 & $0.27 \pm 0.05$ & UNIDENT & \\
\hline N554 & RX J1713.7-3946B & 258.047 & -39.941 & $0.68 \pm 0.06$ & SNR & R 165; !B16; E; \\
\hline N555 & $4 \mathrm{U} 1708-40$ & 258.111 & -40.857 & $0.66 \pm 0.06$ & LMXB & \\
\hline N556 & Oph cluster & 258.113 & -23.349 & $4.10 \pm 0.06$ & CLUSTER & $\mathrm{z}=0.028 ; \mathrm{E}$ \\
\hline N557 & V2400 Oph & 258.150 & -24.242 & $3.37 \pm 0.06$ & $\mathrm{CV}$ & \\
\hline N558 & SAX J1712.6-3739 & 258.159 & -37.642 & $6.55 \pm 0.06$ & LMXB & \\
\hline N559 & IGR J17157-5449 & 258.940 & -54.820 & $0.48 \pm 0.10$ & AGN? & R 166; !B16; \\
\hline N560 & IGR J17158-2124 & 258.960 & -21.410 & $0.43 \pm 0.07$ & UNIDENT & R 53; !B16; \\
\hline N561 & IGR J17162-2117 & 259.070 & -21.290 & $0.47 \pm 0.07$ & UNIDENT & !B16; C; H; \\
\hline N562 & IGR J17163-2155 & 259.088 & -21.917 & $0.33 \pm 0.06$ & UNIDENT & !B16; C; H; \\
\hline N563 & IGR J17164-3803 & 259.120 & -38.060 & $0.89 \pm 0.06$ & CV & R 167; !B16; \\
\hline N564 & NGC 6300 & 259.256 & -62.822 & $5.51 \pm 0.17$ & SEYFERT & $\mathrm{z}=0.004$ \\
\hline N565 & IGR J17174-2436 & 259.360 & -24.610 & $0.70 \pm 0.06$ & UNIDENT & R 168; !B16; \\
\hline N566 & RX J1718.4-4029 & 259.611 & -40.518 & $0.38 \pm 0.06$ & LMXB & !B 16 \\
\hline N567 & IGR J17188-3008 & 259.720 & -30.140 & $0.31 \pm 0.05$ & UNIDENT & !B16; C; \\
\hline N568 & ARP 102B & 259.780 & 49.020 & $2.01 \pm 0.31$ & SEYFERT & $\mathrm{z}=0.024$ \\
\hline N569 & GRS 1716-249 & 259.904 & -25.018 & $1.01 \pm 0.05$ & LMXB & !B16; T; \\
\hline N570 & IGR J17195-4100 & 259.905 & -41.016 & $2.23 \pm 0.06$ & $\mathrm{CV}$ & R 122,110; \\
\hline N571 & IGR J17197-3010 & 259.928 & -30.180 & $0.22 \pm 0.05$ & LMXB & R 169; !B16; \\
\hline N572 & XTE J1720-318 & 259.978 & -31.748 & $0.63 \pm 0.05$ & LMXB & \\
\hline N573 & IGR J17200-3116 & 260.023 & -31.293 & $2.31 \pm 0.05$ & HMXB & R 122,46,76; \\
\hline N574 & IGR J17204-3554 & 260.073 & -35.901 & $0.55 \pm 0.05$ & AGN & R 54,170; \\
\hline N575 & IGR J17217-4557 & 260.430 & -45.960 & $0.35 \pm 0.08$ & UNIDENT & !B 16 \\
\hline N576 & IGR J17227+3411 & 260.690 & 34.200 & $0.77 \pm 0.14$ & AGN? & R 171; !B16; \\
\hline N577 & IGR J17231-3628 & 260.780 & -36.470 & $0.30 \pm 0.05$ & UNIDENT & !B 16 \\
\hline
\end{tabular}


Table A1 - continued from previous page

\begin{tabular}{|c|c|c|c|c|c|c|}
\hline Id & Name $^{1}$ & $\begin{array}{l}\mathrm{RA}^{2} \\
(\mathrm{deg})\end{array}$ & $\begin{array}{l}\operatorname{Dec}^{2} \\
(\mathrm{deg})\end{array}$ & $\begin{array}{c}\text { Flux }^{3} \\
(17-60 \mathrm{keV})\end{array}$ & Type $^{4}$ & Notes \\
\hline N578 & IGR J17233-2837 & 260.849 & -28.619 & $0.81 \pm 0.05$ & SNR / PULSAR & !B16; \\
\hline N579 & IRAS $17216+3633$ & 260.887 & 36.522 & $1.38 \pm 0.14$ & SEYFERT & $\mathrm{z}=0.040$ \\
\hline N580 & EXO 1722-363 & 261.295 & -36.281 & $9.40 \pm 0.05$ & HMXB & \\
\hline N581 & IGR J17254-3257 & 261.350 & -32.963 & $1.76 \pm 0.05$ & LMXB & R 122,172; \\
\hline N582 & IGR J17255-4509 & 261.380 & -45.170 & $0.44 \pm 0.08$ & AGN & R 53; !B16; \\
\hline N583 & $4 \mathrm{U} 1724-30$ & 261.885 & -30.801 & $19.06 \pm 0.05$ & LMXB & \\
\hline N584 & IGR J17285-2922 & 262.171 & -29.382 & $0.22 \pm 0.05$ & LMXB & R 122,173,174; \\
\hline N585 & SWIFT J1728.9-3613 & 262.232 & -36.237 & $1.11 \pm 0.05$ & LMXB? & R 175; !B16; \\
\hline N586 & $1 \mathrm{H} 1726-058$ & 262.589 & -5.981 & $4.35 \pm 0.14$ & $\mathrm{CV}$ & R 122,176; \\
\hline N587 & IGR J17303-2750 & 262.590 & -27.840 & $0.21 \pm 0.05$ & UNIDENT & !B16; \\
\hline N588 & IGR J17306-2015 & 262.650 & -20.260 & $1.32 \pm 0.06$ & UNIDENT & R 168; !B16; \\
\hline N589 & IGR J17315-3221 & 262.890 & -32.360 & $0.21 \pm 0.05$ & UNIDENT & R 168; !B16; \\
\hline N590 & GX 9+9 & 262.937 & -16.955 & $10.48 \pm 0.08$ & LMXB & \\
\hline N591 & GX 354-0 & 262.987 & -33.832 & $57.58 \pm 0.05$ & LMXB & \\
\hline N592 & V2487 Oph & 263.008 & -19.206 & $0.72 \pm 0.07$ & $\mathrm{CV}$ & \\
\hline N593 & GX $1+4$ & 263.011 & -24.743 & $69.52 \pm 0.05$ & LMXB & \\
\hline N594 & IGR J17327-4405 & 263.190 & -44.100 & $0.58 \pm 0.08$ & UNIDENT & R 53; !B16; \\
\hline N595 & IGR J17329-2731 & 263.227 & -27.531 & $0.63 \pm 0.04$ & LMXB & R 177; !B16; \\
\hline N596 & IGR J17331-2406 & 263.248 & -24.143 & $0.38 \pm 0.05$ & LMXB? & R 178,115; T; \\
\hline N597 & Rapid Burster & 263.348 & -33.392 & $3.61 \pm 0.05$ & LMXB & \\
\hline N598 & IGR J17342-4049 & 263.570 & -40.830 & $0.30 \pm 0.06$ & UNIDENT & !B16; \\
\hline N599 & SWIFT J1734.5-3027 & 263.620 & -30.380 & $0.85 \pm 0.04$ & LMXB & R 179; !B16; \\
\hline N600 & IGR J17350-2045 & 263.740 & -20.748 & $0.79 \pm 0.06$ & SEYFERT & R 89,47; z=0.044; \\
\hline N601 & IGR J17350-1957 & 263.750 & -19.960 & $0.32 \pm 0.06$ & UNIDENT & !B16; \\
\hline N602 & IGR J17353-3539 & 263.844 & -35.657 & $0.57 \pm 0.05$ & LMXB & R 180; \\
\hline N603 & IGR J17353-3257 & 263.857 & -32.922 & $1.31 \pm 0.05$ & HMXB & R 181,100,182; \\
\hline N604 & IGR J17361-4444 & 264.040 & -44.730 & $0.61 \pm 0.08$ & LMXB? & R 183,184,185; T; \\
\hline N605 & GRS $1734-292$ & 264.368 & -29.133 & $6.43 \pm 0.04$ & SEYFERT & $\mathrm{z}=0.021$ \\
\hline N606 & ESO 139-G012 & 264.480 & -59.950 & $1.15 \pm 0.16$ & SEYFERT & $\mathrm{z}=0.017$ \\
\hline N607 & IGR J17381-2345 & 264.530 & -23.750 & $0.23 \pm 0.05$ & UNIDENT & !B 16 \\
\hline N608 & SLX 1735-269 & 264.571 & -26.991 & $15.87 \pm 0.04$ & LMXB & \\
\hline N609 & 4U 1735-444 & 264.745 & -44.451 & $21.54 \pm 0.08$ & LMXB & \\
\hline N610 & IGR J17391-3021 & 264.805 & -30.344 & $0.99 \pm 0.04$ & HMXB & R 186,187,188; \\
\hline N611 & GRS 1736-297 & 264.876 & -29.703 & $0.66 \pm 0.04$ & LMXB & \\
\hline N612 & XTE J1739-285 & 264.975 & -28.496 & $1.22 \pm 0.04$ & LMXB & \\
\hline N613 & AX J1740.3-2904 & 265.030 & -29.040 & $0.24 \pm 0.04$ & $\mathrm{CV}$ & \\
\hline N614 & AX J1740.1-2847 & 265.060 & -28.820 & $0.34 \pm 0.04$ & $\mathrm{CV}$ & R 189; !B16; \\
\hline N615 & IGR J17402-3656 & 265.092 & -36.920 & $0.65 \pm 0.05$ & $\mathrm{CV}$ & R 100,77; \\
\hline N616 & SLX 1737-282 & 265.168 & -28.313 & $4.31 \pm 0.04$ & LMXB & \\
\hline N617 & IGR J17407-2808 & 265.175 & -28.133 & $1.88 \pm 0.04$ & HMXB & R 190,191; \\
\hline N618 & IGR J17418-1212 & 265.483 & -12.198 & $2.02 \pm 0.10$ & SEYFERT & R 192,193; z=0.037; \\
\hline N619 & IGR J17422-2108 & 265.560 & -21.140 & $0.24 \pm 0.05$ & SEYFERT & R 11; z=0.106; !B16; \\
\hline N620 & GRS 1739-278 & 265.650 & -27.780 & $2.47 \pm 0.04$ & LMXB & !B16; T; \\
\hline N621 & XTE J1743-363 & 265.748 & -36.380 & $1.29 \pm 0.05$ & HMXB & R 194; \\
\hline N622 & PKS 1741-03 & 265.970 & -3.788 & $0.59 \pm 0.12$ & BLAZAR & $\mathrm{z}=1.054$ \\
\hline N623 & 1E $1740.7-294$ & 265.984 & -29.735 & $50.02 \pm 0.04$ & LMXB & \\
\hline N624 & GRO J1744-28 & 266.138 & -28.741 & $3.80 \pm 0.04$ & LMXB & \\
\hline N625 & IGR J17446-2947 & 266.174 & -29.805 & $12.13 \pm 0.04$ & LMXB & R 195,196,197; T; C; \\
\hline N626 & IGR J17449-3037 & 266.230 & -30.620 & $0.21 \pm 0.04$ & UNIDENT & !B 16 \\
\hline N627 & KS $1741-293$ & 266.242 & -29.337 & $6.40 \pm 0.04$ & LMXB & $\mathrm{T}$ \\
\hline N628 & GRS 1741.9-2853 & 266.250 & -28.917 & $4.45 \pm 0.04$ & LMXB & R 198,103; T; \\
\hline N629 & SWIFT J174510.8-262411 & 266.295 & -26.404 & $0.48 \pm 0.04$ & LMXB? & R 199; !B16; T; \\
\hline N630 & IGR J17456-2901 & 266.401 & -29.026 & $6.99 \pm 0.04$ & GAL CENTER & R 200,201,202; SGR A*; C; \\
\hline N631 & SWIFT J1745.4+2906 & 266.460 & 29.140 & $1.01 \pm 0.22$ & SEYFERT & R 203; z=0.111; !B16; \\
\hline N632 & A $1742-294$ & 266.525 & -29.518 & $11.77 \pm 0.04$ & LMXB & R 204; T; \\
\hline N633 & IGR J17464-3213 & 266.564 & -32.234 & $25.26 \pm 0.04$ & LMXB & R 205,206; T; \\
\hline N634 & 1E $1743.1-2843$ & 266.580 & -28.735 & $6.14 \pm 0.04$ & LMXB & \\
\hline N635 & SAX J1747.0-2853 & 266.761 & -28.883 & $4.45 \pm 0.04$ & LMXB & R 207,208; T; \\
\hline
\end{tabular}


Table A1 - continued from previous page

\begin{tabular}{|c|c|c|c|c|c|c|}
\hline Id & Name $^{1}$ & $\begin{array}{l}\mathrm{RA}^{2} \\
(\mathrm{deg})\end{array}$ & $\begin{array}{l}\operatorname{Dec}^{2} \\
(\mathrm{deg})\end{array}$ & $\begin{array}{c}\text { Flux }^{3} \\
(17-60 \mathrm{keV})\end{array}$ & Type $^{4}$ & Notes \\
\hline N636 & IGR J17464-2811 & 266.817 & -28.180 & $1.37 \pm 0.04$ & LMXB & R 209,210,211,212,208; T; \\
\hline N637 & SLX 1744-299/300 & 266.834 & -30.010 & $8.94 \pm 0.04$ & LMXB & $\mathrm{T}$ \\
\hline N638 & IGR J17473-2721 & 266.838 & -27.348 & $1.78 \pm 0.04$ & LMXB & R 213,214,215,216,217; T; \\
\hline N639 & IGR J17475-2822 & 266.868 & -28.370 & $2.12 \pm 0.04$ & MOL CLOUD & R 218,219,220; \\
\hline N640 & IGR J17475-2253 & 266.886 & -22.872 & $0.86 \pm 0.05$ & SEYFERT & $\mathrm{R} 87,98,221 ; \mathrm{z}=0.047$ \\
\hline N641 & IGR J17479-2807 & 266.982 & -28.121 & $1.06 \pm 0.04$ & LMXB & R 14,52 \\
\hline N642 & GX $3+1$ & 266.984 & -26.556 & $11.59 \pm 0.04$ & LMXB & \\
\hline N643 & IGR J17488-2338 & 267.150 & -23.590 & $0.22 \pm 0.05$ & SEYFERT & $\mathrm{R} 67 ; \mathrm{z}=0.240$ \\
\hline N644 & 4U $1745-203$ & 267.208 & -20.361 & $0.97 \pm 0.06$ & LMXB & $\mathrm{T}$ \\
\hline N645 & IGR J17488-3253 & 267.218 & -32.920 & $1.37 \pm 0.04$ & SEYFERT & R $122,46,222 ; z=0.020$ \\
\hline N646 & AX J1749.1-2733 & 267.275 & -27.550 & $0.98 \pm 0.04$ & HMXB & R 223,224; T; \\
\hline N647 & AX J1749.2-2725 & 267.292 & -27.421 & $0.54 \pm 0.04$ & HMXB & R 225,226,227; \\
\hline N648 & GRO J1750-27 & 267.300 & -26.647 & $4.29 \pm 0.04$ & HMXB & R 228,229; T; \\
\hline N649 & IGR J17497-2821 & 267.396 & -28.353 & $1.89 \pm 0.04$ & LMXB & R 230,231,232; \\
\hline N650 & IGR J17498-2921 & 267.482 & -29.323 & $0.53 \pm 0.04$ & LMXB & R 233,234,235,236,237,238; !B16; T; \\
\hline N651 & $4 \mathrm{U} 1746-37$ & 267.552 & -37.046 & $2.44 \pm 0.05$ & LMXB & \\
\hline N652 & SAX J1750.8-2900 & 267.600 & -29.038 & $2.25 \pm 0.04$ & LMXB & $\mathrm{C}$ \\
\hline N653 & IGR J17505-2644 & 267.658 & -26.734 & $0.69 \pm 0.04$ & LMXB? & R 239,240; \\
\hline N654 & IGR J17507-2856 & 267.683 & -28.922 & $1.43 \pm 0.04$ & UNIDENT & R 241,3,242; T; C; \\
\hline N655 & GRS 1747-312 & 267.691 & -31.282 & $1.45 \pm 0.04$ & LMXB & $\mathrm{R} 243 ; \mathrm{T}$ \\
\hline N656 & IGR J17513-2011 & 267.824 & -20.197 & $1.28 \pm 0.06$ & SEYFERT & R 54,46; z=0.047; \\
\hline N657 & SWIFT J1751.8-6019 & 267.968 & -60.322 & $1.14 \pm 0.17$ & SEYFERT & $\mathrm{R} 22 ; \mathrm{z}=0.112$ \\
\hline N658 & XTE J1752-223 & 268.044 & -22.325 & $0.86 \pm 0.05$ & LMXB & \\
\hline N659 & IGR J17528-2022 & 268.210 & -20.370 & $0.31 \pm 0.06$ & CV? & R 244; !B16; \\
\hline N660 & SWIFT J1753.5-0127 & 268.368 & -1.452 & $81.97 \pm 0.11$ & LMXB & R 245,246; T; \\
\hline N661 & AX J1753.5-2745 & 268.375 & -27.750 & $0.21 \pm 0.04$ & UNIDENT & $\mathrm{R} 77 ;$ !B16; \\
\hline N662 & SAX J1753.5-2349 & 268.400 & -23.810 & $0.21 \pm 0.05$ & LMXB & $\mathrm{R} 247$ \\
\hline N663 & IGR J17538-2544 & 268.460 & -25.750 & $0.20 \pm 0.04$ & UNIDENT & R 248,249,250; !B16; T; H; \\
\hline N664 & AX J1754.2-2754 & 268.574 & -27.902 & $0.29 \pm 0.04$ & LMXB & R 251,247; !B16; \\
\hline N665 & IGR J17544-2619 & 268.601 & -26.321 & $0.76 \pm 0.04$ & HMXB & R 252,253; T; \\
\hline N666 & IGR J17554-1832 & 268.860 & -18.550 & $0.29 \pm 0.06$ & UNIDENT & !B16; \\
\hline N667 & IGR J17570-2500 & 269.270 & -25.020 & $0.38 \pm 0.05$ & UNIDENT & R 53; !B16; H; \\
\hline N668 & IGR J17585-3057 & 269.615 & -30.960 & $0.56 \pm 0.04$ & LMXB? & R 254; \\
\hline N669 & IGR J17586-2129 & 269.656 & -21.372 & $1.80 \pm 0.05$ & CV? & R 100,69,76,77,76; \\
\hline N670 & IGR J17591-2342 & 269.787 & -23.713 & $0.96 \pm 0.05$ & LMXB & R 255,256; !B16; T; \\
\hline N671 & IGR J17596-2315 & 269.910 & -23.270 & $0.47 \pm 0.05$ & UNIDENT & R $53 ;$ !B16; \\
\hline N672 & IGR J17597-2201 & 269.939 & -22.019 & $2.40 \pm 0.05$ & LMXB & R 257,258,135,208,76; \\
\hline N673 & IGR J17597-1935 & 269.940 & -19.600 & $0.29 \pm 0.06$ & UNIDENT & !B 16 \\
\hline N674 & IGR J17598-2236 & 269.950 & -22.600 & $0.29 \pm 0.05$ & UNIDENT & !B16; \\
\hline N675 & NGC 6552 & 270.030 & 66.615 & $0.71 \pm 0.12$ & SEYFERT & $\mathrm{z}=0.027$ \\
\hline N676 & IGR J18006-3426 & 270.170 & -34.450 & $0.25 \pm 0.05$ & UNIDENT & !B16; \\
\hline N677 & V2301 OPH & 270.170 & 8.181 & $1.47 \pm 0.16$ & $\mathrm{CV}$ & \\
\hline N678 & IGR J18007-4146 & 270.230 & -41.810 & $0.79 \pm 0.08$ & $\mathrm{CV}$ & R 111,56,259; \\
\hline N679 & IGR J18010-3045 & 270.270 & -30.760 & $0.37 \pm 0.04$ & UNIDENT & R 53,93; !B16; \\
\hline N680 & GX 5-1 & 270.286 & -25.078 & $48.68 \pm 0.05$ & LMXB & \\
\hline N681 & GRS 1758-258 & 270.302 & -25.743 & $74.70 \pm 0.05$ & LMXB & \\
\hline N682 & GX 9+1 & 270.387 & -20.517 & $17.21 \pm 0.06$ & LMXB & $\mathrm{C}$ \\
\hline N683 & IGR J18017-3542 & 270.440 & -35.710 & $2.16 \pm 0.05$ & CV? & R 53,93; !B16; \\
\hline N684 & IGR J18027-2016 & 270.677 & -20.278 & $5.33 \pm 0.06$ & HMXB & R 260,261; T; C; \\
\hline N685 & IGR J18027-1455 & 270.692 & -14.907 & $2.14 \pm 0.07$ & SEYFERT & R 122,$262 ; z=0.036$ \\
\hline N686 & 1RXS J180408.9-342058 & 271.038 & -34.349 & $3.57 \pm 0.05$ & LMXB & R 263; !B16; T; \\
\hline N687 & IGR J18044-2739 & 271.110 & -27.660 & $0.77 \pm 0.04$ & $\mathrm{CV}$ & R 264,265; \\
\hline N688 & IGR J18044-1829 & 271.110 & -18.490 & $0.46 \pm 0.06$ & UNIDENT & R 11; !B16; \\
\hline N689 & IGR J18048-1455 & 271.177 & -14.942 & $0.76 \pm 0.07$ & $\mathrm{CV}$ & R 54,89; \\
\hline N690 & SAX J1806.5-2215 & 271.642 & -22.252 & $2.72 \pm 0.05$ & LMXB & R 266,267,268,212; !B16; \\
\hline N691 & XTE J1807-294 & 271.744 & -29.416 & $0.58 \pm 0.05$ & LMXB & $\mathrm{T}$ \\
\hline N692 & IGR J18070-3507 & 271.760 & -35.130 & $0.30 \pm 0.05$ & UNIDENT & R 53; !B16; \\
\hline N693 & IGR J18078+1123 & 271.946 & 11.381 & $1.22 \pm 0.17$ & SEYFERT & R $15 ; z=0.079$ \\
\hline
\end{tabular}


Table A1 - continued from previous page

\begin{tabular}{|c|c|c|c|c|c|c|}
\hline Id & Name $^{1}$ & $\begin{array}{l}\mathrm{RA}^{2} \\
(\mathrm{deg})\end{array}$ & $\begin{array}{l}\operatorname{Dec}^{2} \\
(\mathrm{deg})\end{array}$ & $\begin{array}{c}\text { Flux }^{3} \\
(17-60 \mathrm{keV})\end{array}$ & Type $^{4}$ & Notes \\
\hline N694 & V426 Oph & 271.973 & 5.815 & $0.56 \pm 0.13$ & $\mathrm{CV}$ & !B16; \\
\hline N695 & SAX J1808.4-3658 & 272.115 & -36.979 & $0.56 \pm 0.06$ & LMXB & $\mathrm{T}$ \\
\hline N696 & SGR 1806-20 & 272.165 & -20.411 & $2.45 \pm 0.06$ & SNR / PULSAR & \\
\hline N697 & IGR J18088-2741 & 272.210 & -27.700 & $0.67 \pm 0.05$ & $\mathrm{CV}$ & R 167,114; !B16; \\
\hline N698 & IGR J18102-1751 & 272.560 & -17.850 & $0.65 \pm 0.07$ & UNIDENT & R 53; !B16; \\
\hline N699 & XTE J1810-189 & 272.585 & -19.070 & $2.97 \pm 0.06$ & LMXB & $\mathrm{T}$ \\
\hline N700 & SAX J1810.8-2609 & 272.685 & -26.150 & $1.82 \pm 0.05$ & LMXB & \\
\hline N701 & IGR J18109-2631 & 272.740 & -26.520 & $0.42 \pm 0.05$ & UNIDENT & R 53; !B16; C; \\
\hline N702 & IGR J18112-2641 & 272.820 & -26.690 & $0.47 \pm 0.05$ & CV? & R 53,93; !B16; \\
\hline N703 & PSR J1811-1925 & 272.862 & -19.420 & $1.16 \pm 0.06$ & SNR / PULSAR & \\
\hline N704 & MAXI J1810-222 & 273.170 & -22.310 & $0.39 \pm 0.06$ & LMXB & R 269; !B16; T; \\
\hline N705 & IGR J18134-1636 & 273.364 & -16.618 & $0.76 \pm 0.07$ & AGN? & R 208; \\
\hline N706 & MAXI J1813-095 & 273.392 & -9.533 & $0.36 \pm 0.08$ & LMXB & R 270,271,272; !B16; \\
\hline N707 & IGR J18135-1751 & 273.397 & -17.841 & $1.76 \pm 0.07$ & SNR / PULSAR & R 273,274; \\
\hline N708 & IGR J18141-1823 & 273.540 & -18.390 & $0.51 \pm 0.07$ & AGN? & R 11; !B16; \\
\hline N709 & IGR J18141-0606 & 273.550 & -6.110 & $0.46 \pm 0.08$ & UNIDENT & R 53; !B16; \\
\hline N710 & GX $13+1$ & 273.629 & -17.155 & $13.09 \pm 0.07$ & LMXB & \\
\hline N711 & IGR J18147-3400 & 273.690 & -34.010 & $0.56 \pm 0.05$ & UNIDENT & R 53; !B16; \\
\hline N712 & IGR J18149-2247 & 273.740 & -22.800 & $0.26 \pm 0.06$ & UNIDENT & !B16; \\
\hline N713 & $4 \mathrm{U} 1812-12$ & 273.777 & -12.094 & $35.46 \pm 0.08$ & LMXB & \\
\hline N714 & IGR J18151-1052 & 273.778 & -10.879 & $0.54 \pm 0.08$ & $\mathrm{CV}$ & R 156,275,276; \\
\hline N715 & GX $17+2$ & 274.006 & -14.035 & $62.73 \pm 0.07$ & LMXB & \\
\hline N716 & AM Her & 274.041 & 49.872 & $6.18 \pm 0.54$ & $\mathrm{CV}$ & \\
\hline N717 & IGR J18165-3912 & 274.140 & -39.200 & $0.63 \pm 0.08$ & $\mathrm{CV}$ ? & R 78; !B16; \\
\hline N718 & SWIFT J1816.7-1613 & 274.141 & -16.252 & $0.29 \pm 0.07$ & HMXB & R 277,278; T; \\
\hline N719 & IGR J18170-2511 & 274.310 & -25.152 & $1.37 \pm 0.05$ & $\mathrm{CV}$ & R 7; \\
\hline N720 & IGR J18172-1944 & 274.310 & -19.740 & $0.50 \pm 0.06$ & UNIDENT & R 53; !B16; T; \\
\hline N721 & XTE J1817-330 & 274.431 & -33.019 & $3.40 \pm 0.05$ & LMXB & $\mathrm{T}$ \\
\hline N722 & XTE J1818-245 & 274.606 & -24.528 & $3.18 \pm 0.05$ & LMXB & \\
\hline N723 & IGR J18184-2352 & 274.610 & -23.880 & $0.88 \pm 0.06$ & CV? & R 53; !B16; \\
\hline N724 & SAX J1818.6-1703 & 274.679 & -17.041 & $1.60 \pm 0.07$ & HMXB & R 1; T; \\
\hline N725 & IGR J18193-2542 & 274.880 & -25.680 & $0.64 \pm 0.05$ & UNIDENT & R 3; !B16; \\
\hline N726 & MAXI J1820+070 & 275.091 & 7.185 & $16.25 \pm 0.11$ & LMXB & R 279; !B16; T; \\
\hline N727 & AX J1820.5-1434 & 275.133 & -14.564 & $1.41 \pm 0.07$ & HMXB & \\
\hline N728 & IGR J18214-1318 & 275.327 & -13.316 & $1.68 \pm 0.08$ & HMXB & R 54,280,281,282; T; \\
\hline N729 & IGR J18219-1347 & 275.498 & -13.792 & $0.74 \pm 0.08$ & HMXB & R 89,283 \\
\hline N730 & H $1821+643$ & 275.506 & 64.369 & $1.53 \pm 0.13$ & SEYFERT & \\
\hline N731 & $4 \mathrm{U} 1820-30$ & 275.918 & -30.365 & $35.61 \pm 0.06$ & LMXB & \\
\hline N732 & IC 4709 & 276.086 & -56.376 & $2.85 \pm 0.18$ & SEYFERT & $\mathrm{z}=0.017$ \\
\hline N733 & XTE J1824-141 & 276.115 & -14.438 & $0.88 \pm 0.08$ & HMXB? & R 284,77; \\
\hline N734 & IGR J18245-2452 & 276.140 & -24.880 & $1.02 \pm 0.06$ & LMXB & R 285; !B16; T; \\
\hline N735 & IGR J18249-3243 & 276.225 & -32.734 & $1.02 \pm 0.06$ & SEYFERT & R 286,7,287; z=0.355; \\
\hline N736 & $4 \mathrm{U} 1823-00$ & 276.335 & 0.000 & $1.91 \pm 0.09$ & LMXB & \\
\hline N737 & IGR J18256-1035 & 276.443 & -10.589 & $0.75 \pm 0.08$ & HMXB & R 3,67,77,208; \\
\hline N738 & 4U $1822-371$ & 276.445 & -37.106 & $29.71 \pm 0.07$ & LMXB & \\
\hline N739 & IGR J18257-0707 & 276.486 & -7.155 & $0.95 \pm 0.08$ & SEYFERT & R 54,3; z=0.037; \\
\hline N740 & IGR J18263-1345 & 276.550 & -13.694 & $0.55 \pm 0.08$ & UNIDENT & R 53; !B16; \\
\hline N741 & LS 5039 & 276.557 & -14.855 & $1.09 \pm 0.08$ & HMXB & \\
\hline N742 & IGR J18284-0229 & 277.110 & -2.480 & $0.37 \pm 0.08$ & UNIDENT & R 52; \\
\hline N743 & MAXI J1828-249 & 277.243 & -25.030 & $1.06 \pm 0.06$ & LMXB & R 288,289; !B16; T; \\
\hline N744 & IGR J18293-1213 & 277.340 & -12.222 & $0.59 \pm 0.08$ & CV? & R 290,63,89; \\
\hline N745 & GS $1826-24$ & 277.370 & -23.798 & $93.74 \pm 0.06$ & LMXB & \\
\hline N746 & IGR J18303-3345 & 277.592 & -33.752 & $0.55 \pm 0.07$ & UNIDENT & !B16; C; H; \\
\hline N747 & AX J1830.6-1002 & 277.650 & -10.047 & $0.81 \pm 0.08$ & AGN? & R 291; \\
\hline N748 & IGR J18308-1232 & 277.698 & -12.531 & $0.85 \pm 0.08$ & CV & R 100; \\
\hline N749 & IGR J18308+0928 & 277.710 & 9.480 & $0.47 \pm 0.09$ & SEYFERT & R 15; z=0.019; \\
\hline N750 & IGR J18312-3334 & 277.820 & -33.580 & $0.63 \pm 0.07$ & SEYFERT & R 52,15; \\
\hline N751 & AX J1832.3-0840 & 278.109 & -8.660 & $0.69 \pm 0.08$ & $\mathrm{CV}$ & \\
\hline
\end{tabular}


Table A1 - continued from previous page

\begin{tabular}{|c|c|c|c|c|c|c|}
\hline Id & Name $^{1}$ & $\begin{array}{l}\mathrm{RA}^{2} \\
(\mathrm{deg})\end{array}$ & $\begin{array}{l}\operatorname{Dec}^{2} \\
(\mathrm{deg})\end{array}$ & $\begin{array}{c}\text { Flux }^{3} \\
(17-60 \mathrm{keV})\end{array}$ & Type $^{4}$ & Notes \\
\hline N752 & IGR J18325-0756 & 278.110 & -7.944 & $1.19 \pm 0.08$ & HMXB? & R 292,3; \\
\hline N753 & SNR 021.5-00.9 & 278.388 & -10.583 & $4.00 \pm 0.08$ & SNR / PULSAR & \\
\hline N754 & PKS 1830-211 & 278.421 & -21.056 & $3.07 \pm 0.07$ & BLAZAR & $\mathrm{z}=2.507$ \\
\hline N755 & 3C 382 & 278.783 & 32.698 & $4.13 \pm 0.30$ & SEYFERT & $\mathrm{z}=0.058$ \\
\hline N756 & MAXI J1836-194 & 278.931 & -19.320 & $2.51 \pm 0.08$ & LMXB & R 293; !B16; T; \\
\hline N757 & RX J1832-33 & 278.931 & -32.993 & $7.94 \pm 0.07$ & LMXB & \\
\hline N758 & FAIRALL 49 & 279.210 & -59.410 & $1.04 \pm 0.19$ & SEYFERT & $\mathrm{z}=0.020$ \\
\hline N759 & AX J1838.0-0655 & 279.505 & -6.918 & $2.74 \pm 0.08$ & SNR / PULSAR & R 294; \\
\hline N760 & IGR J18381-0924 & 279.534 & -9.414 & $0.66 \pm 0.08$ & SEYFERT? & R 167,$114 ; z=0.031$ \\
\hline N761 & ESO 103-G035 & 279.576 & -65.425 & $6.16 \pm 0.22$ & SEYFERT & $\mathrm{z}=0.013$ \\
\hline N762 & SWIFT J1839.1-5717 & 279.761 & -57.296 & $1.03 \pm 0.19$ & UNIDENT & R 56,295; \\
\hline N763 & Ser X-1 & 279.991 & 5.041 & $11.28 \pm 0.07$ & LMXB & \\
\hline N764 & IGR J18400-2439 & 280.020 & -24.660 & $0.33 \pm 0.08$ & UNIDENT & R 168; !B16; \\
\hline N765 & IGR J18410-0535 & 280.255 & -5.573 & $1.11 \pm 0.08$ & HMXB & R 296,297; \\
\hline N766 & 1E $1841-045$ & 280.333 & -4.941 & $3.47 \pm 0.08$ & MAGNETAR & \\
\hline N767 & 3C 390.3 & 280.582 & 79.768 & $5.01 \pm 0.21$ & SEYFERT & $\mathrm{z}=0.056$ \\
\hline N768 & IGR J18434-0508 & 280.860 & -5.140 & $0.49 \pm 0.08$ & CV? & R 53,93; !B16; \\
\hline N769 & ESO 140-G043 & 281.212 & -62.376 & $2.83 \pm 0.20$ & SEYFERT & $\mathrm{z}=0.014$ \\
\hline N770 & AX J1845.0-0433 & 281.267 & -4.568 & $1.73 \pm 0.08$ & HMXB & \\
\hline N771 & GS $1843+00$ & 281.404 & 0.872 & $3.84 \pm 0.07$ & HMXB & $\mathrm{T}$ \\
\hline N772 & SWIFT J1845.7-0037 & 281.478 & -0.659 & $0.42 \pm 0.07$ & HMXB & R 298,299,300,301; !B16; \\
\hline N773 & IGR J18462-0223 & 281.567 & -2.387 & $0.31 \pm 0.07$ & HMXB & R 302,303,304,305; \\
\hline N774 & PSR J1846-0258 & 281.610 & -2.977 & $2.25 \pm 0.08$ & SNR / PULSAR & R 306; \\
\hline N775 & H $1846-786$ & 281.779 & -78.560 & $1.16 \pm 0.22$ & SEYFERT & $\mathrm{z}=0.074 ; \mathrm{B} 16$ \\
\hline N776 & 1A $1845-024$ & 282.062 & -2.443 & $2.15 \pm 0.07$ & HMXB & $\mathrm{T}$ \\
\hline N777 & IGR J18483-0311 & 282.071 & -3.172 & $5.74 \pm 0.08$ & HMXB & R $307,145,1$; \\
\hline N778 & IGR J18486-0047 & 282.097 & -0.777 & $1.30 \pm 0.07$ & AGN? & R 100 \\
\hline N779 & IGR J18490-0000 & 282.264 & -0.020 & $1.36 \pm 0.07$ & SNR / PULSAR & R 308,309; \\
\hline N780 & EXO 1846-031 & 282.321 & -3.062 & $0.65 \pm 0.08$ & LMXB & !B16; C; \\
\hline N781 & IGR J18497-0248 & 282.446 & -2.804 & $0.57 \pm 0.07$ & UNIDENT & R 310,77; !B16; \\
\hline N782 & 4U 1850-08 & 283.270 & -8.706 & $6.83 \pm 0.08$ & LMXB & \\
\hline N783 & IGR J18538-0102 & 283.459 & -1.034 & $0.57 \pm 0.07$ & SEYFERT & R 35; z=0.145; \\
\hline N784 & IGR J18544+0839 & 283.610 & 8.660 & $0.50 \pm 0.07$ & AGN? & R 11; !B16; \\
\hline N785 & ESO 025-G002 & 283.752 & -78.891 & $1.15 \pm 0.22$ & SEYFERT & $\mathrm{z}=0.029 ; \mathrm{C}$ \\
\hline N786 & 4U 1849-31 & 283.762 & -31.162 & $7.85 \pm 0.10$ & $\mathrm{CV}$ & \\
\hline N787 & XTE J1855-026 & 283.876 & -2.604 & $13.01 \pm 0.07$ & HMXB & $\mathrm{T}$ \\
\hline N788 & IGR J18559+1535 & 283.990 & 15.635 & $1.67 \pm 0.08$ & SEYFERT & R 311; z=0.084; \\
\hline N789 & XTE J1856+053 & 284.179 & 5.310 & $0.32 \pm 0.06$ & LMXB & $\mathrm{T}$ \\
\hline N790 & 2E $1849.2-7832$ & 284.388 & -78.491 & $1.97 \pm 0.22$ & SEYFERT & $\mathrm{z}=0.042$ \\
\hline N791 & SWIFT J1858.6-0814 & 284.645 & -8.237 & $0.74 \pm 0.09$ & X-RAY BINARY & !B16; T; \\
\hline N792 & XTE J1858+034 & 284.693 & 3.430 & $5.72 \pm 0.06$ & HMXB & $\mathrm{T}$ \\
\hline N793 & XTE J1859+083 & 284.775 & 8.250 & $0.60 \pm 0.06$ & HMXB & !B16; \\
\hline N794 & HETE J19001-2455 & 285.036 & -24.920 & $22.99 \pm 0.12$ & LMXB & R 312; T; \\
\hline N795 & XTE J1901+014 & 285.419 & 1.444 & $3.85 \pm 0.07$ & LMXB? & R 313,314,261; T; \\
\hline N796 & $4 \mathrm{U} 1901+03$ & 285.914 & 3.215 & $12.96 \pm 0.06$ & HMXB & $\mathrm{T}$ \\
\hline N797 & IGR J19039+3348 & 285.985 & 33.815 & $1.14 \pm 0.15$ & SEYFERT & R 33; z=0.015; !B16; \\
\hline N798 & IGR J19071+0716 & 286.780 & 7.270 & $0.25 \pm 0.06$ & UNIDENT & R 53; !B16; \\
\hline N799 & V1082 Sgr & 286.807 & -20.765 & $1.14 \pm 0.13$ & CV? & R 315; \\
\hline N800 & SGR $1900+14$ & 286.848 & 9.322 & $1.09 \pm 0.06$ & MAGNETAR & \\
\hline N801 & IGR J19077-3925 & 286.900 & -39.378 & $0.87 \pm 0.11$ & SEYFERT & R $15 ; z=0.073$ \\
\hline N802 & XTE J1908+094 & 287.221 & 9.385 & $0.58 \pm 0.06$ & LMXB & \\
\hline N803 & SWIFT J1909.3+0124 & 287.340 & 1.200 & $0.34 \pm 0.07$ & $\mathrm{CV}$ & R 316,317; !B16; \\
\hline N804 & 4U $1907+09$ & 287.407 & 9.832 & $13.66 \pm 0.06$ & HMXB & $\mathrm{T}$ \\
\hline N805 & 4U 1909+07 & 287.701 & 7.599 & $17.82 \pm 0.06$ & HMXB & \\
\hline N806 & Aql X-1 & 287.814 & 0.584 & $10.06 \pm 0.07$ & LMXB & $\mathrm{T}$ \\
\hline N807 & IGR J19113+1413 & 287.830 & 14.220 & $0.79 \pm 0.07$ & $\mathrm{CV}$ & R 77,318; !B16; \\
\hline N808 & SS 433 & 287.954 & 4.983 & $10.59 \pm 0.06$ & HMXB & \\
\hline N809 & IGR J19140+0951 & 288.518 & 9.884 & $11.28 \pm 0.06$ & HMXB & R 319,261; \\
\hline
\end{tabular}


Table A1 - continued from previous page

\begin{tabular}{|c|c|c|c|c|c|c|}
\hline Id & Name $^{1}$ & $\begin{array}{l}\mathrm{RA}^{2} \\
(\mathrm{deg})\end{array}$ & $\begin{array}{l}\operatorname{Dec}^{2} \\
(\operatorname{deg})\end{array}$ & $\begin{array}{c}\text { Flux }^{3} \\
(17-60 \mathrm{keV})\end{array}$ & Type $^{4}$ & Notes \\
\hline $\mathrm{N} 810$ & GRS $1915+105$ & 288.798 & 10.945 & $315.96 \pm 0.06$ & LMXB & \\
\hline N811 & IGR J19173+0747 & 289.330 & 7.830 & $0.75 \pm 0.06$ & CV? & R 167,320; \\
\hline N812 & 4U $1915-05$ & 289.699 & -5.237 & $10.73 \pm 0.10$ & LMXB & \\
\hline N813 & IGR J19193+0754 & 289.820 & 7.908 & $0.28 \pm 0.06$ & UNIDENT & R 321; !B16; \\
\hline N814 & IGR J19194-2956 & 289.863 & -29.959 & $1.00 \pm 0.13$ & SEYFERT & R 15; z=0.167; \\
\hline N815 & ABELL 2319 & 290.260 & 43.974 & $1.35 \pm 0.15$ & CLUSTER & \\
\hline N816 & ESO141-G055 & 290.302 & -58.678 & $3.79 \pm 0.23$ & SEYFERT & $\mathrm{z}=0.037$ \\
\hline N817 & SWIFT J1922.7-1716 & 290.601 & -17.297 & $0.72 \pm 0.14$ & LMXB & R 322,323; \\
\hline N818 & IGR J19225+1836 & 290.640 & 18.610 & $0.40 \pm 0.08$ & UNIDENT & !B16; \\
\hline N819 & PKS 1921-293 & 291.220 & -29.246 & $1.01 \pm 0.14$ & BLAZAR & $\mathrm{z}=0.352$ \\
\hline N820 & IGR J19260+4136 & 291.578 & 41.586 & $0.55 \pm 0.12$ & SEYFERT & R 22; $\mathrm{z}=0.071 ; \mathrm{B} 16$ \\
\hline N821 & SWIFT J1926.4-0501 & 291.581 & -5.020 & $0.46 \pm 0.10$ & AGN & R 38; !B16; \\
\hline N822 & IGR J19267+1325 & 291.646 & 13.396 & $0.55 \pm 0.07$ & $\mathrm{CV}$ & R 324; \\
\hline N823 & IGR J19294+1328 & 292.360 & 13.470 & $0.69 \pm 0.07$ & AGN & R 111; \\
\hline N824 & IGR J19295-0225 & 292.390 & -2.420 & $0.61 \pm 0.10$ & UNIDENT & !B16; \\
\hline N825 & IGR J19294+1816 & 292.483 & 18.311 & $1.47 \pm 0.08$ & HMXB & R 325; \\
\hline N826 & IGR J19302+3411 & 292.525 & 34.174 & $1.20 \pm 0.09$ & SEYFERT & R 24; $z=0.063$ \\
\hline N827 & SNR G054.1+00.3 & 292.630 & 18.860 & $0.76 \pm 0.08$ & SNR / PULSAR & \\
\hline N828 & SWIFT J1933.9+3258 & 293.437 & 32.915 & $1.43 \pm 0.09$ & SEYFERT & R 326,$31 ; z=0.058$ \\
\hline N829 & SWIFT J1937.5-4021 & 294.310 & -40.270 & $0.50 \pm 0.10$ & UNIDENT & R 38; !B16; \\
\hline N830 & 1H $1934-063$ & 294.405 & -6.227 & $1.55 \pm 0.13$ & SEYFERT & $\mathrm{z}=0.010$ \\
\hline N831 & RX J1940.2-1025 & 295.056 & -10.423 & $3.51 \pm 0.15$ & $\mathrm{CV}$ & \\
\hline N832 & IGR J19405-3016 & 295.065 & -30.268 & $1.43 \pm 0.14$ & SEYFERT & R $1 ; z=0.052$ \\
\hline N833 & IGR J19421+3613 & 295.530 & 36.220 & $0.38 \pm 0.08$ & UNIDENT & R 53; !B16; \\
\hline N834 & NGC 6814 & 295.674 & -10.325 & $4.10 \pm 0.15$ & SEYFERT & $\mathrm{z}=0.005$ \\
\hline N835 & IGR J19443+2117 & 296.023 & 21.305 & $0.95 \pm 0.09$ & BLAZAR? & R 100; \\
\hline N836 & XTE J1946+274 & 296.414 & 27.365 & $3.06 \pm 0.09$ & HMXB & $\mathrm{T}$ \\
\hline N837 & XSS J19459+4508 & 296.806 & 44.823 & $1.63 \pm 0.10$ & SEYFERT & R 70,45; z=0.054; \\
\hline N838 & IGR J19491-1035 & 297.280 & -10.590 & $1.01 \pm 0.16$ & SEYFERT & R 41; z=0.024; \\
\hline N839 & KS $1947+300$ & 297.396 & 30.212 & $8.65 \pm 0.08$ & HMXB & \\
\hline N840 & AX J1949.8+2534 & 297.467 & 25.573 & $0.64 \pm 0.09$ & HMXB & R 327; !B16; \\
\hline N841 & IGR J19504+3318 & 297.620 & 33.310 & $1.09 \pm 0.07$ & AGN? & R 53,93; !B16; \\
\hline N842 & $3 \mathrm{C} 403$ & 298.096 & 2.504 & $0.98 \pm 0.11$ & SEYFERT & $\mathrm{z}=0.058$ \\
\hline N843 & PSR B1951+32 & 298.250 & 32.930 & $1.08 \pm 0.07$ & SNR / PULSAR & \\
\hline N844 & IGR J19552+0044 & 298.800 & 0.770 & $0.70 \pm 0.13$ & $\mathrm{CV}$ & R 328; \\
\hline N845 & $4 \mathrm{U} 1954+31$ & 298.925 & 32.097 & $12.52 \pm 0.07$ & LMXB & R 329; \\
\hline N846 & IGR J19577+3339 & 299.440 & 33.650 & $0.43 \pm 0.07$ & AGN? & R 53,93; !B16; \\
\hline N847 & V2306 Cyg & 299.570 & 32.551 & $1.19 \pm 0.07$ & $\mathrm{CV}$ & \\
\hline N848 & Cyg X-1 & 299.590 & 35.204 & $867.58 \pm 0.07$ & HMXB & \\
\hline N849 & $4 \mathrm{U} 1957+11$ & 299.809 & 11.713 & $1.87 \pm 0.11$ & LMXB & \\
\hline N850 & Cyg A & 299.869 & 40.739 & $6.49 \pm 0.07$ & SEYFERT & $\mathrm{z}=0.056$ \\
\hline N851 & 1ES $1959+650$ & 299.999 & 65.149 & $1.33 \pm 0.19$ & BLAZAR & $\mathrm{z}=0.047$ \\
\hline N852 & SWIFT J2000.6+3210 & 300.093 & 32.187 & $1.72 \pm 0.07$ & HMXB & R 330; \\
\hline N853 & IGR J20063+3641 & 301.576 & 36.698 & $0.58 \pm 0.07$ & $\mathrm{CV}$ & R 318,244; !B16; \\
\hline N854 & ESO 399-IG 020 & 301.755 & -34.534 & $1.14 \pm 0.13$ & SEYFERT & $\mathrm{z}=0.025$ \\
\hline N855 & IGR J20084+3221 & 302.120 & 32.350 & $0.69 \pm 0.07$ & CV? & R 53,93; !B16; \\
\hline N856 & NGC 6860 & 302.184 & -61.086 & $2.41 \pm 0.36$ & SEYFERT & $\mathrm{z}=0.015$ \\
\hline N857 & IGR J20107+4534 & 302.680 & 45.570 & $0.65 \pm 0.08$ & UNIDENT & R 114; !B16; \\
\hline N858 & IGR J20159+3713 & 303.946 & 37.213 & $1.05 \pm 0.07$ & SNR & R 318,331; C; \\
\hline N859 & IGR J20166+7603 & 304.170 & 76.060 & $1.26 \pm 0.26$ & UNIDENT & !B16; \\
\hline N860 & IGR J20169+2714 & 304.230 & 27.230 & $0.42 \pm 0.09$ & UNIDENT & !B16; \\
\hline N861 & PKS 2014-55 & 304.466 & -55.629 & $2.08 \pm 0.42$ & SEYFERT & R 22; z=0.060; \\
\hline N862 & IGR J20187+4041 & 304.642 & 40.694 & $1.75 \pm 0.07$ & SEYFERT & R 286,332; z=0.014; \\
\hline N863 & IGR J20216+4359 & 305.428 & 44.012 & $1.00 \pm 0.07$ & SEYFERT & R 333; z=0.017; \\
\hline N864 & V404 Cyg & 306.016 & 33.867 & $41.78 \pm 0.07$ & HMXB & !B16; T; \\
\hline N865 & IGR J20286+2544 & 307.143 & 25.746 & $3.20 \pm 0.11$ & SEYFERT & R 286,334,335; z=0.014; AGN pair; \\
\hline N866 & IGR J20310+3835 & 307.755 & 38.576 & $0.54 \pm 0.07$ & AGN? & R 111,56; \\
\hline N867 & EXO 2030+375 & 308.062 & 37.638 & $59.10 \pm 0.07$ & HMXB & $\mathrm{T}$ \\
\hline
\end{tabular}


Table A1 - continued from previous page

\begin{tabular}{|c|c|c|c|c|c|c|}
\hline Id & Name $^{1}$ & $\begin{array}{l}\mathrm{RA}^{2} \\
(\mathrm{deg})\end{array}$ & $\begin{array}{l}\operatorname{Dec}^{2} \\
(\mathrm{deg})\end{array}$ & $\begin{array}{c}\text { Flux }^{3} \\
(17-60 \mathrm{keV})\end{array}$ & Type $^{4}$ & Notes \\
\hline N868 & Cyg X-3 & 308.108 & 40.959 & $181.74 \pm 0.07$ & HMXB & \\
\hline N869 & $4 \mathrm{C}+21.55$ & 308.405 & 21.791 & $1.63 \pm 0.16$ & BLAZAR & $\mathrm{z}=0.174$ \\
\hline N870 & SWIFT J2037.2+4151 & 309.252 & 41.833 & $0.60 \pm 0.07$ & UNIDENT & R 77,336,337; \\
\hline N871 & $4 \mathrm{C}+74.26$ & 310.661 & 75.126 & $3.51 \pm 0.30$ & SEYFERT & $\mathrm{z}=0.104$ \\
\hline N872 & RX J2044.0+2833 & 311.004 & 28.550 & $1.08 \pm 0.11$ & SEYFERT & $\mathrm{z}=0.049$ \\
\hline N873 & MRK 509 & 311.043 & -10.731 & $5.16 \pm 0.17$ & SEYFERT & $\mathrm{z}=0.034$ \\
\hline N874 & IC 5063 & 312.943 & -57.056 & $3.99 \pm 0.76$ & SEYFERT & R 22; z=0.011; !B16; \\
\hline N875 & SWIFT J2055.0+3559 & 313.770 & 35.950 & $0.38 \pm 0.08$ & UNIDENT & R $38 ;$ !B 16 \\
\hline N876 & IGR J20569+4940 & 314.178 & 49.660 & $0.94 \pm 0.08$ & BLAZAR & R 97,77; \\
\hline N877 & IGR J20596+4303 & 314.956 & 43.058 & $0.46 \pm 0.08$ & UNIDENT & R 22,78; !B16; C; \\
\hline N878 & SAX J2103.5+4545 & 315.897 & 45.750 & $10.44 \pm 0.08$ & HMXB & $\mathrm{T}$ \\
\hline N879 & IGR J21060-4447 & 316.520 & -44.780 & $1.58 \pm 0.31$ & UNIDENT & !B16; \\
\hline N880 & RX J2109.4+3532 & 317.490 & 35.560 & $0.56 \pm 0.10$ & BLAZAR? & $\mathrm{z}=0.202 ; ! \mathrm{B} 16$ \\
\hline N881 & IGR J21133+3154 & 318.340 & 31.947 & $0.55 \pm 0.13$ & STAR & !B16; \\
\hline N882 & 1RXS J211336.1+542226 & 318.375 & 54.368 & $0.46 \pm 0.09$ & CV & R 15; \\
\hline N883 & S5 $2116+81$ & 318.624 & 82.077 & $2.16 \pm 0.39$ & SEYFERT & $\mathrm{z}=0.086$ \\
\hline N884 & IGR J21178+5139 & 319.464 & 51.650 & $1.51 \pm 0.09$ & BLAZAR? & R 286,338; z=0.002; \\
\hline N885 & IGR J21196+3333 & 319.899 & 33.552 & $1.16 \pm 0.13$ & SEYFERT & R $15 ; z=0.051$ \\
\hline N886 & RX J2123.7+4217 & 320.920 & 42.296 & $1.33 \pm 0.09$ & $\mathrm{CV}$ & \\
\hline N887 & IGR J21247+5058 & 321.163 & 50.975 & $10.56 \pm 0.09$ & SEYFERT & R 122,$262 ; z=0.015$ \\
\hline N888 & IGR J21277+5656 & 321.922 & 56.937 & $2.92 \pm 0.10$ & SEYFERT & R $311 ; z=0.015$ \\
\hline N889 & $4 U 2129+12$ & 322.489 & 12.175 & $4.81 \pm 0.40$ & LMXB & \\
\hline N890 & RX J2133.7+5107 & 323.433 & 51.132 & $3.77 \pm 0.09$ & $\mathrm{CV}$ & R 123; \\
\hline N891 & IGR J21358+4729 & 323.976 & 47.486 & $1.49 \pm 0.09$ & SEYFERT & $\mathrm{z}=0.025$ \\
\hline N892 & IGR J21382+3204 & 324.582 & 32.036 & $1.13 \pm 0.21$ & SEYFERT & $\mathrm{R} 22 ; \mathrm{z}=0.025 ; \mathrm{B} 16$ \\
\hline N893 & IGR J21397+5949 & 324.905 & 59.848 & $0.83 \pm 0.11$ & SEYFERT & R $15 ; z=0.114$ \\
\hline N894 & SS Cyg & 325.688 & 43.577 & $3.43 \pm 0.10$ & $\mathrm{CV}$ & \\
\hline N895 & Cyg X-2 & 326.170 & 38.319 & $28.13 \pm 0.14$ & LMXB & \\
\hline N896 & PKS 2149-306 & 327.981 & -30.465 & $3.69 \pm 0.18$ & BLAZAR & R 339; z=2.345; \\
\hline N897 & MRK 520 & 330.124 & 10.547 & $2.18 \pm 0.39$ & SEYFERT & R 340; z=0.027; \\
\hline N898 & IGR J22018+5049 & 330.471 & 50.810 & $0.80 \pm 0.10$ & BLAZAR & R 22; z=1.899; !B16; \\
\hline N899 & NGC 7172 & 330.490 & -31.877 & $7.55 \pm 0.18$ & SEYFERT & $\mathrm{z}=0.009$ \\
\hline N900 & BL Lac & 330.672 & 42.281 & $1.35 \pm 0.14$ & BLAZAR & $\mathrm{z}=0.069$ \\
\hline N901 & $4 U 2206+54$ & 331.987 & 54.518 & $9.99 \pm 0.10$ & HMXB & $\mathrm{T}$ \\
\hline N902 & FO Aqr & 334.481 & -8.351 & $2.68 \pm 0.48$ & $\mathrm{CV}$ & \\
\hline N903 & SWIFT J2221.6+5952 & 335.540 & 59.880 & $0.47 \pm 0.09$ & UNIDENT & R 38; !B16; \\
\hline N904 & IGR J22292+6646 & 337.324 & 66.778 & $0.89 \pm 0.09$ & SEYFERT & R $15 ; z=0.112$ \\
\hline N905 & NGC 7314 & 338.940 & -26.061 & $3.34 \pm 0.25$ & SEYFERT & $\mathrm{z}=0.005$ \\
\hline N906 & MRK 915 & 339.155 & -12.531 & $2.34 \pm 0.32$ & SEYFERT & $\mathrm{z}=0.024 ;$ !B16; \\
\hline N907 & 3C 452 & 341.383 & 39.683 & $2.21 \pm 0.44$ & SEYFERT & $\mathrm{z}=0.081$ \\
\hline N908 & IGR J22516+5610 & 342.900 & 56.180 & $0.38 \pm 0.08$ & UNIDENT & !B16; \\
\hline N909 & IGR J22517+2218 & 342.939 & 22.316 & $1.30 \pm 0.20$ & BLAZAR & R $341 ; z=3.668$ \\
\hline N910 & IGR J22534+6243 & 343.350 & 62.720 & $0.58 \pm 0.07$ & HMXB & R 342,343,344; !B16; \\
\hline N911 & 3C 454.3 & 343.490 & 16.146 & $9.01 \pm 0.17$ & BLAZAR & $\mathrm{z}=0.859$ \\
\hline N912 & MR 2251-178 & 343.507 & -17.604 & $1.96 \pm 0.28$ & SEYFERT & $\mathrm{z}=0.064$ \\
\hline N913 & AX J2254.3+1146 & 343.610 & 11.760 & $0.83 \pm 0.19$ & SEYFERT & $\mathrm{z}=0.028 ;$ !B16; \\
\hline N914 & AO Psc & 343.837 & -3.164 & $1.97 \pm 0.43$ & $\mathrm{CV}$ & \\
\hline N915 & Kaz 320 & 344.874 & 24.917 & $1.94 \pm 0.23$ & SEYFERT & $\mathrm{z}=0.035$ \\
\hline N916 & NGC 7465 & 345.508 & 15.953 & $1.35 \pm 0.17$ & SEYFERT & $\mathrm{z}=0.007$ \\
\hline N917 & NGC 7469 & 345.837 & 8.870 & $4.34 \pm 0.23$ & SEYFERT & $\mathrm{z}=0.016$ \\
\hline N918 & MRK 926 & 346.176 & -8.685 & $4.10 \pm 0.32$ & SEYFERT & $\mathrm{z}=0.047$ \\
\hline N919 & NGC 7479 & 346.236 & 12.323 & $1.65 \pm 0.19$ & SEYFERT & $\mathrm{z}=0.008$ \\
\hline N920 & NGC 7582 & 349.597 & -42.370 & $5.48 \pm 0.59$ & SEYFERT & $\mathrm{z}=0.005$ \\
\hline N921 & IGR J23206+6431 & 350.163 & 64.535 & $0.80 \pm 0.06$ & SEYFERT & R $333 ; z=0.072$ \\
\hline N922 & Cas A & 350.859 & 58.800 & $4.31 \pm 0.06$ & SNR & \\
\hline N923 & RX J2325.9+2153 & 351.503 & 21.922 & $1.78 \pm 0.25$ & SEYFERT & $\mathrm{z}=0.120$ \\
\hline N924 & PKS 2325+093 & 351.933 & 9.597 & $1.38 \pm 0.30$ & BLAZAR & $\mathrm{z}=1.840$ \\
\hline N925 & IGR J23300+8406 & 352.500 & 84.100 & $2.90 \pm 0.59$ & UNIDENT & !B16; \\
\hline
\end{tabular}


Table A1 - continued from previous page

\begin{tabular}{|c|c|c|r|c|c|l|}
\hline Id & Name $^{1}$ & \multicolumn{1}{c|}{$\begin{array}{c}\text { RA }^{2} \\
(\mathrm{deg})\end{array}$} & $\begin{array}{c}\text { Dec } \\
(\mathrm{deg})\end{array}$ & $\begin{array}{c}\text { Flux }^{3} \\
(17-60 \mathrm{keV})\end{array}$ & Type $^{4}$ & \multicolumn{1}{c|}{ Notes } \\
\hline N926 & IGR J23308+7120 & 352.632 & 71.415 & $0.64 \pm 0.09$ & SEYFERT & R 1,345,346; z=0.037; \\
N927 & IGR J23439-2148 & 355.990 & -21.800 & $3.11 \pm 0.57$ & UNIDENT & !B16; \\
N928 & 1ES 2344+514 & 356.760 & 51.700 & $0.47 \pm 0.10$ & BLAZAR & z=0.044; !B16; \\
N929 & IGR J23523+5844 & 358.065 & 58.763 & $0.64 \pm 0.06$ & SEYFERT & R 333,25,1,58,346; z=0.164;
\end{tabular}

\section{APPENDIX B: REFERENCES IN THE CATALOG}

(1) Masetti et al. (2008), (2) den Hartog et al. (2006), (3) Tomsick et al. (2008), (4) Kuiper et al. (2006b), (5) Eckert et al. (2004), (6) Markwardt et al. (2004), (7) Masetti et al. (2009), (8) den Hartog et al. (2004b), (9) Coe et al. (2015), (10) Rojas et al. (2017), (11) Karasev et al. (2018), (12) Townsend et al. (2011), (13) Burenin et al. (2006b), (14) Bird et al. (2010), (15) Masetti et al. (2010b), (16) Tomsick et al. (2012), (17) Segreto et al. (2013), (18) Halpern \& Tyagi (2005), (19) Wang (2010), (20) Rodriguez et al. (2010), (21) Kuiper et al. (2005), (22) Baumgartner et al. (2013), (23) Watson et al. (2009), (24) Burenin et al. (2008), (25) Rodriguez et al. (2008), (26) Bikmaev et al. (2017), (27) Beri et al. (2021), (28) Doroshenko et al. (2020a), (29) Apparao et al. (1978), (30) Baumgartner et al. (2010), (31) Parisi et al. (2012), (32) Bikmaev et al. (2008b), (33) Parisi et al. (2014), (34) Lutovinov et al. (2010b), (35) Lutovinov et al. (2012), (36) Burenin et al. (2006a), (37) Mereminskiy et al. (2016), (38) Oh et al. (2018), (39) Tomsick et al. (2015), (40) Spiro et al. (2013), (41) Krivonos et al. (2011), (42) Martí et al. (2004), (43) Grebenev et al. (2013), (44) Bernardini et al. (2015), (45) Sazonov et al. (2005), (46) Masetti et al. (2006c), (47) Malizia et al. (2016), (48) Vasilopoulos et al. (2014), (49) Vasilopoulos et al. (2013), (50) Revnivtsev et al. (2007), (51) Winter et al. (2008), (52) Bird et al. (2016), (53) Krivonos et al. (2017), (54) Bird et al. (2006), (55) den Hartog et al. (2004a), (56) Landi et al. (2017), (57) Malizia et al. (2012), (58) Sazonov et al. (2008), (59) Revnivtsev et al. (2009), (60) Bernardini et al. (2012), (61) Zurita Heras et al. (2009), (62) Sanna et al. (2017), (63) Landi et al. (2010b), (64) Parisi et al. (2008), (65) Huchra et al. (2012), (66) Tueller et al. (2008), (67) Masetti et al. (2013), (68) Kuiper et al. (2006a), (69) Coleiro et al. (2013), (70) Revnivtsev et al. (2006), (71) Masetti et al. (2006d), (72) Morelli et al. (2006), (73) Masetti et al. (2006a), (74) Leyder et al. (2008), (75) Fiocchi et al. (2010), (76) Fortin et al. (2018), (77) Clavel et al. (2019), (78) Karasev et al. (2020), (79) Bird et al. (2007), (80) Lubiński et al. (2005), (81) Negueruela et al. (2005), (82) Sidoli et al. (2006), (83) Produit et al. (2004), (84) Grebenev et al. (2004), (85) Negueruela et al. (2007), (86) González-Martín et al. (2011), (87) Mescheryakov et al. (2009), (88) Masetti et al. (2010a), (89) Karasev et al. (2012), (90) Krivonos et al. (2018), (91) Oh et al. (2015), (92) Malizia et al. (2017), (93) Tomsick et al. (2021), (94) Chernyakova et al. (2005), (95) Aharonian et al. (2005), (96) Sazonov et al. (2007), (97) Landi et al. (2010a), (98) Krivonos et al. (2007b), (99) Kniazev et al. (2010), (100) Tomsick et al. (2009), (101) D’Aì et al. (2011), (102) Koss et al. (2011), (103) Cornelisse et al. (2002), (104) Keek et al. (2006), (105) Renaud et al. (2010), (106) Landi et al. (2011a), (107) Tomsick et al. (2016a), (108) Bernardini et al. (2018), (109) Molina et al. (2015), (110) Masetti et al. (2006e), (111) Tomsick et al. (2020), (112) Tuerler et al. (2012), (113) Tueller et al. (2010), (114) Tomsick et al. (2016b), (115) Malizia et al. (2010), (116) Milisavljevic et al. (2011), (117) Krimm et al. (2011), (118) Masetti et al. (2007c), (119) Edelson \& Malkan (2012), (120) Landi et al. (2011b), (121) Margutti et al. (2019), (122) Walter et al. (2004), (123) Barlow et al. (2006), (124) Masetti et al. (2007b), (125) Tomsick et al. (2006), (126) Soldi et al. (2005), (127) Beckmann et al. (2005), (128) Kuiper et al. (2008), (129) Ratti et al. (2010), (130) Miyasaka et al. (2018), (131) Revnivtsev et al. (2003a), (132) Courvoisier et al. (2003), (133) Tomsick et al. (2003), (134) Lutovinov et al. (2005a), (135) Walter et al. (2006), (136) Negueruela \& Schurch (2007), (137) Acero et al. (2015), (138) Revnivtsev et al. (2003c), (139) Nespoli et al. (2010), (140) Bodaghee et al. (2006), (141) Tomsick et al. (2004), (142) Lutovinov et al. (2004b), (143) Lutovinov et al. (2005b), (144) Molkov et al. (2003), (145) Chaty et al. (2008), (146) Grebenev et al. (2005b), (147) Nespoli et al. (2008b), (148) Pearlman et al. (2019), (149) Koss et al. (2017), (150) Hiemstra et al. (2011), (151) Lutovinov et al. (2010a), (152) Burenin et al. (2007), (153) Xiao et al. (2019), (154) Grebenev et al. (2018), (155) Kalamkar et al. (2011), (156) Krivonos et al. (2009), (157) Sanchez-Fernandez et al. (2015), (158) Churazov et al. (2007), (159) Degenaar et al. (2012), (160) Kuulkers et al. (2003), (161) Lutovinov \& Revnivtsev (2003), (162) Capitanio et al. (2006), (163) Grebenev et al. (2005a), (164) Grebenev et al. (2007a), (165) Kuznetsova et al. (2019), (166) Landi et al. (2012), (167) Rahoui et al. (2017), (168) Krivonos et al. (2012), (169) Masetti et al. (2012a), (170) Bassani et al. (2005), (171) Caccianiga et al. (2008), (172) Brandt et al. (2006a), (173) Barlow et al. (2005), (174) Sidoli et al. (2011), (175) Barthelmy et al. (2019), (176) Gänsicke et al. (2005), (177) Bozzo et al. (2018a), (178) Lutovinov et al. (2004a), (179) Bozzo et al. (2015), (180) Degenaar et al. (2010), (181) Kuulkers et al. (2006), (182) Goossens et al. (2019), (183) Gibaud et al. (2011), (184) Nucita et al. (2012), (185) Del Santo et al. (2014), (186) Sunyaev et al. (2003a), (187) Smith \& Hartigan (2006), (188) Sguera et al. (2020), (189) Britt et al. (2013), (190) Kretschmar et al. (2004), (191) Heinke et al. (2009), (192) Bassani et al. (2004), (193) Torres et al. (2004), (194) Falanga et al. (2011), (195) Krivonos et al. (2013), (196) Bahramian

\footnotetext{
1 Name of the source. The sources newly discovered in the hard X-ray domain are highlighted in bold text.

2 Equatorial coordinates (right ascension and declination) are in the standard J2000.0 epoch.

3 The measured 17-60 keV flux of the source $\times 10^{-11} \mathrm{erg} \mathrm{cm}^{-2} \mathrm{~s}^{-1}$. The flux is highlighted in red colour if the source is detected at $S / N<4.5 \sigma$.

4 General astrophysical type of the object. A question mark indicates that the specified type should be confirmed.

5 This column contains notes on the identification and classification of some sources and letter-coded information flags as follows: "R" - comma-separated list of references, which can be found in Sect. B; "z" - redshift; "T" - transient source; "E" - spatially extended source; "C" - spatial confusion with other source, measured flux should be taken with caution; " $\mathrm{H}$ " - the source is located in a region with high systematic noise, and its measured flux should be taken with caution; "!B16" - the source has not been found in the INTEGRAL/IBIS survey by Bird et al. (2016).
} 
et al. (2014), (197) Heinke et al. (2019), (198) Cocchi et al. (1999), (199) Vovk et al. (2012), (200) Bélanger et al. (2006), (201) Krivonos et al. (2007a), (202) Mori et al. (2015), (203) Mescheryakov et al. (2006), (204) Pavlinsky et al. (1994), (205) Revnivtsev et al. (2003b), (206) Kalemci et al. (2006), (207) in 't Zand et al. (1998), (208) Zolotukhin \& Revnivtsev (2015), (209) Brandt et al. (2006b), (210) Wijnands (2006), (211) Degenaar et al. (2007), (212) Kaur et al. (2017), (213) Grebenev et al. (2005c), (214) Del Monte et al. (2008b), (215) Altamirano et al. (2008), (216) Del Monte et al. (2008a), (217) Zhang et al. (2009), (218) Revnivtsev et al. (2004b), (219) Terrier et al. (2010), (220) Zhang et al. (2015), (221) Rodriguez et al. (2009), (222) Malizia et al. (2007), (223) Grebenev \& Sunyaev (2007), (224) Zurita Heras \& Chaty (2008), (225) Karasev et al. (2010), (226) Kaur et al. (2010), (227) Nebot Gómez-Morán et al. (2015), (228) Scott et al. (1997), (229) Brandt et al. (2008), (230) Soldi et al. (2006), (231) Paizis et al. (2007), (232) Rodriguez et al. (2007), (233) Papitto et al. (2011), (234) Ferrigno et al. (2011), (235) Bozzo et al. (2011), (236) Greiss et al. (2011), (237) Russell et al. (2011), (238) Chakrabarty et al. (2011b), (239) Zolotukhin \& Revnivtsev (2011), (240) Revnivtsev et al. (2008), (241) Grebenev \& Sunyaev (2004), (242) Natalucci et al. (2011), (243) Grebenev et al. (2019), (244) Hare et al. (2021), (245) Morgan et al. (2005), (246) Neustroev et al. (2014), (247) Shaw et al. (2017), (248) Kuulkers et al. (2013), (249) Krimm et al. (2013b), (250) Krimm et al. (2013a), (251) Chelovekov \& Grebenev (2007), (252) Sunyaev et al. (2003b), (253) in't Zand (2005), (254) Curran et al. (2011), (255) Ducci et al. (2018), (256) Sanna et al. (2018), (257) Lutovinov et al. (2003b), (258) Brandt et al. (2007), (259) Coughenour et al., (in prep.), (260) Revnivtsev et al. (2004a), (261) Torrejón et al. (2010), (262) Masetti et al. (2004), (263) Chenevez et al. (2012), (264) Nucita et al. (2007), (265) Masetti et al. (2012b), (266) Del Santo et al. (2011), (267) Chakrabarty et al. (2011a), (268) Kaur et al. (2011), (269) Negoro et al. (2018), (270) Fuerst et al. (2018), (271) Rau (2018), (272) Armas Padilla et al. (2019), (273) Ubertini et al. (2005), (274) Funk et al. (2007), (275) Burenin et al. (2009), (276) Worpel et al. (2020), (277) Nabizadeh et al. (2019), (278) Halpern \& Gotthelf (2008), (279) Bozzo et al. (2018b), (280) Butler et al. (2009), (281) Fornasini et al. (2017), (282) Cusumano et al. (2020), (283) La Parola et al. (2013), (284) Markwardt (2008), (285) Eckert et al. (2013), (286) Bassani et al. (2006), (287) Landi et al. (2009), (288) Nakahira et al. (2013), (289) Oda et al. (2019), (290) Clavel et al. (2016), (291) Bassani et al. (2009), (292) Lutovinov et al. (2003a), (293) Ferrigno et al. (2012), (294) Malizia et al. (2005), (295) Pavlinsky et al. (2021), (296) Rodriguez et al. (2004), (297) Nespoli et al. (2008a), (298) Doroshenko et al. (2020b), (299) Krimm et al. (2012), (300) McCollum \& Laine (2019), (301) Kennea et al. (2019), (302) Grebenev et al. (2007b), (303) Grebenev \& Sunyaev (2010), (304) Bodaghee et al. (2012), (305) Sguera et al. (2013), (306) Kuiper \& Hermsen (2009), (307) Chernyakova et al. (2003), (308) Molkov et al. (2004), (309) Terrier et al. (2008), (310) Reynolds et al. (2012), (311) Bikmaev et al. (2006), (312) Kaaret et al. (2006), (313) Karasev et al. (2007), (314) Karasev et al. (2008), (315) Xu et al. (2019), (316) Stephen et al. (2018), (317) Halpern \& Thorstensen (2018), (318) Halpern et al. (2018), (319) Hannikainen et al. (2003), (320) Pavan et al. (2011), (321) Krivonos et al. (2010b), (322) Tueller et al. (2005), (323) Degenaar et al. (2011), (324) Steeghs et al. (2008), (325) Rodes-Roca et al. (2018), (326) Torres et al. (2006), (327) Hare et al. (2019), (328) Tovmassian et al. (2017), (329) Masetti et al. (2007a), (330) Halpern (2006), (331) Bassani et al. (2014), (332) Goncalves et al. (2008), (333) Bikmaev et al. (2008a), (334) Masetti et al. (2006b), (335) Winter et al. (2009), (336) Westergaard et al. (2006), (337) Landi et al. (2011c), (338) Maselli et al. (2013), (339) Gianní et al. (2011), (340) Lamperti et al. (2017), (341) Bassani et al. (2007), (342) Masetti et al. (2012c), (343) Esposito et al. (2013), (344) Lutovinov et al. (2013), (345) Landi et al. (2007), (346) de Rosa et al. (2012). 
$30 \quad$ Krivonos et al.

This paper has been typeset from a $\mathrm{T}_{\mathrm{E}} \mathrm{X} / \mathrm{L} \mathrm{T} \mathrm{E} \mathrm{X}$ file prepared by the author. 